\title{
REMOTE SENSING OF SALT MARSH VEGETATION STRESS
}

Bas Frank Oteman 



\title{
REMOTE SENSING OF SALT MARSH VEGETATION STRESS
}

\author{
DISSERTATION
}

\author{
to obtain \\ the degree of doctor at the Universiteit Twente, \\ on the authority of the rector magnificus, \\ prof. dr. ir. A. Veldkamp, \\ on account of the decision of the Doctorate Board \\ to be publicly defended \\ on Thursday 18 March 2021 at 12.45 hours
}

by

\section{Bas Frank Oteman}

born on the 4th of August, 1988

in Arnhem, The Netherlands 
This dissertation has been approved by:

Supervisors

Prof. dr. D. van der Wal

Prof. dr. T.J. Bouma

Cover design: Job Duim

Printed by: CTRL-P

Lay-out: Bas Oteman

ISBN: 978-90-365-5135-9

DOI: $10.3990 / 1.9789036551359$

(C) 2021 Bas Frank Oteman, The Netherlands. All rights reserved. No parts of this thesis may be reproduced, stored in a retrieval system or transmitted in any form or by any means without permission of the author. Alle rechten voorbehouden. Niets uit deze uitgave mag worden vermenigvuldigd, in enige vorm of op enige wijze, zonder voorafgaande schriftelijke toestemming van de auteur. 


\section{Graduation Committee:}

Chair / secretary:

Supervisors:

Committee Members: prof.dr. F.D. van der Meer

prof.dr. D. van der Wal prof.dr. T.J. Bouma

prof.dr. A.K. Skidmore dr.ir. C. van der Tol prof. dr. S. Temmerman prof. dr. S.M. de Jong dr. S. Nolte 


\section{Acknowledgements}

I would like to acknowledge nature organization Het Zeeuwse Landschap for allowing us to use their nature reserve as field site. I would like to thank Lennart van IJzerloo, Jeroen van Dalen, Adriana Constantinescu and Eline ten Dolle for their large contribution to the field work. We would also like to thank Annette Wielemaker for her help processing the data.

I want to thank all NIOZ colleagues who helped with numerous things. I want to especially thank Laura, Hélène, Sil, Jim, Greg and Roeland for their help with fieldwork, various analyses and most importantly their mental support and encouragement to keep going.

This project has received funding from the European Union's Seventh Framework Programme (Space) under grant agreement no 607131, project FAST (Foreshore Assessment using Space Technology). The team behind this project was fundamental to enabling this research, and helped me in many ways. I want to thank Ed, Ben and Albert in particular, for their help in overcoming many problems and their positive attitude.

Lastly I want to thank my supervisors, Daphne in particular, her patience and critical feedback helped improve the quality of this thesis, and helped me become a better scientist. 


\section{Table of Contents}

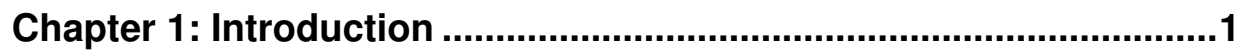

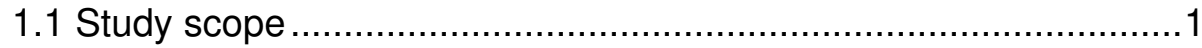

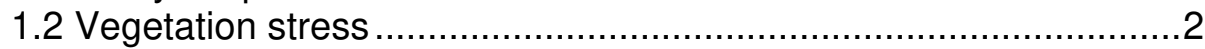

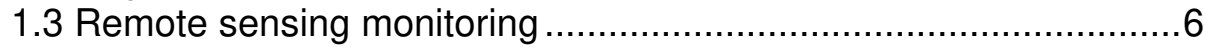

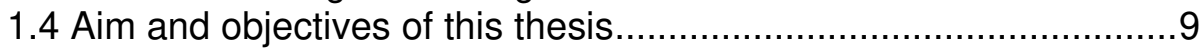

\section{Chapter 2: Depth from focus with a regular camera to analyze} small scale habitat structure .............................................................11

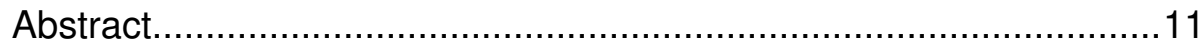

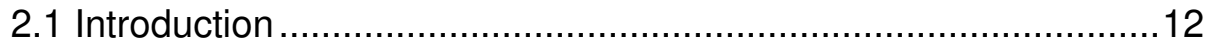

2.2 Materials and methods ........................................................ 15

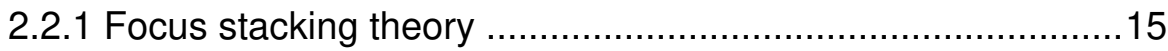

2.2.2 Camera setup and stack preprocessing ..............................15

2.2.3 Lens calibration and focus distance estimation .....................16

2.2.4 Validation of distance estimation in the laboratory .................18

2.2.5 Validation of seed thickness in the laboratory ....................... 18

2.2.6 Validation of saltmarsh vegetation structure in the laboratory 18

2.2.7 Application to saltmarsh vegetation structure in the field .......19

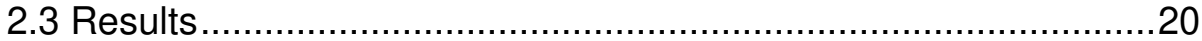

2.3.1 Validation of distance estimation in the laboratory ................20

2.3.2 Validation of seed thickness in the laboratory .......................21

2.3.3 Validation of saltmarsh vegetation structure in the laboratory 21

2.3.4 Application to saltmarsh vegetation structure in the field .......24

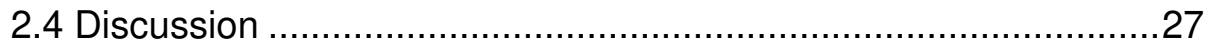

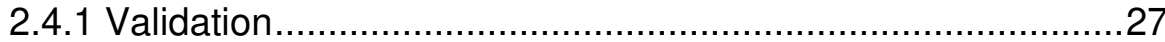

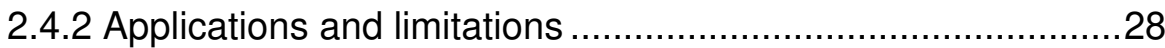

2.4.3 Possible improvements and developments .........................29

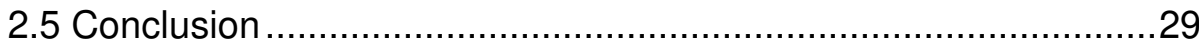

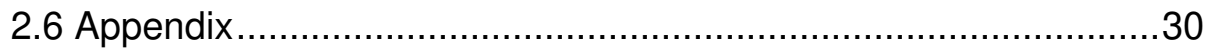


Chapter 3: Using remote sensing to identify drivers behind spatial patterns in the bio-physical properties of a saltmarsh pioneer .......31

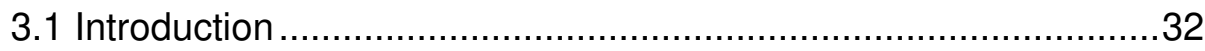

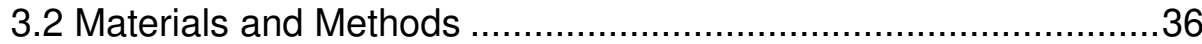

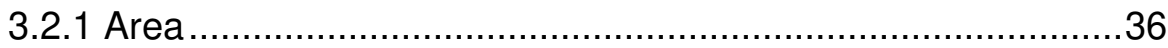

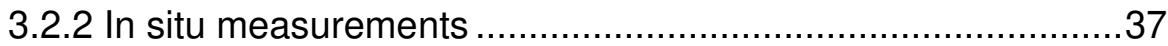

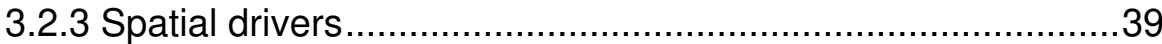

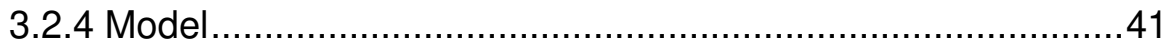

3.2.5 Model inversion ........................................................... 43

3.2.6 Sensitivity modeled vegetation characteristics .......................44

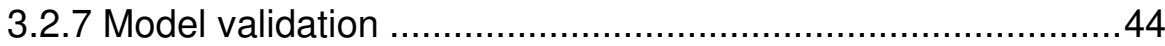

3.2.8 Application to space borne data ..........................................44

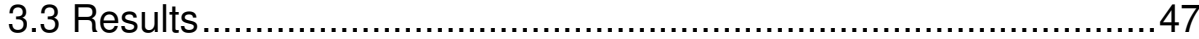

3.3.1 Effects of spatial drivers on in situ vegetation characteristics 47

3.3.2 Effects of vegetation characteristics on reflectance,

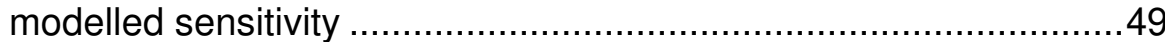

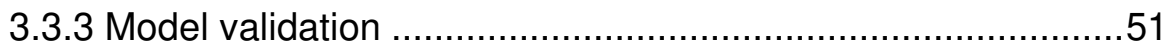

3.3.4 Large scale effect of spatial drivers .....................................53

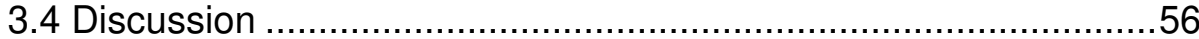

3.4.1 Applicability to other vegetation zones ................................57

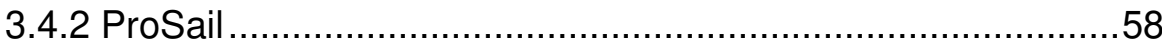

3.4.3 Effect of spatial drivers on leaf and canopy level ..................60

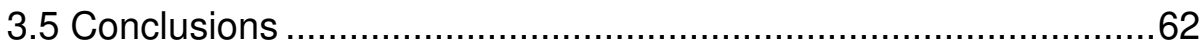

\section{Chapter 4: Indicators of expansion and retreat of Phragmites} based on optical and radar satellite remote sensing: a case study

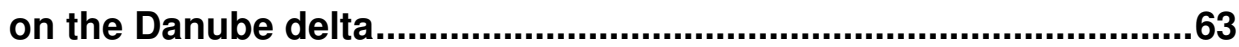

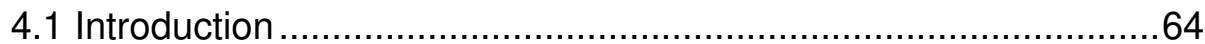

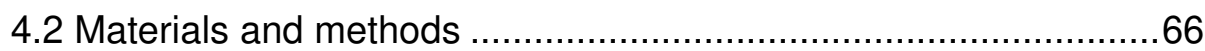

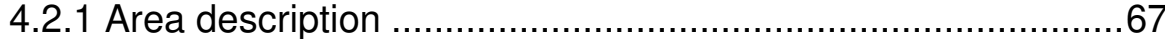

4.2.2 Seasonal in situ measurements of reed characteristics .........67

4.2.3 Categorizing long-term reed development from

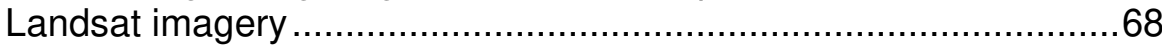


4.2.4 Establishing wave exposure of reeds, using fetch length from Landsat imagery.

4.2.5 Establishing long-term reed development from Landsat imagery....

4.2.6 Analyzing seasonal remote sensing indicators of long-term

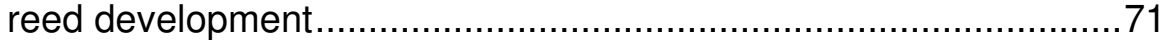

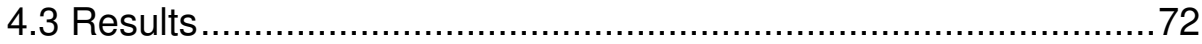

4.3.1 Long-term reed development from remote sensing................72

4.3.2 Seasonal remote sensing as indicators of long-term

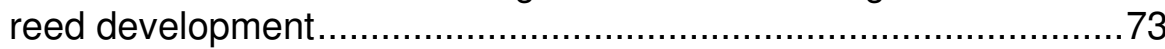

4.3.3 Relating seasonal remote sensing indicators to in situ patterns

4.3.4 Seasonal variation in properties of reed vegetation

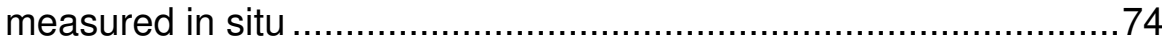

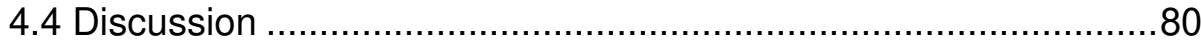

4.4.1 Seasonal remote sensing as indicators of long-term reed

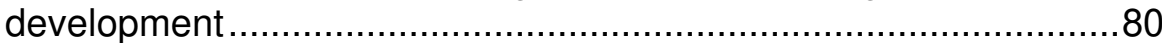

4.4.2 Long-term reed development from remote sensing................82

4.4.3 Conclusions and outlook ..................................................83

Chapter 5: Stress in salt marshes at leaf, plant and community level; towards predicting ecosystem development from satellite

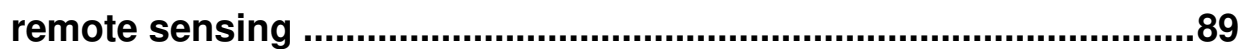

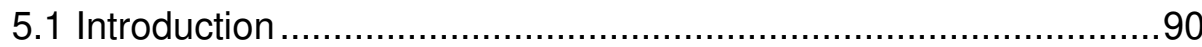

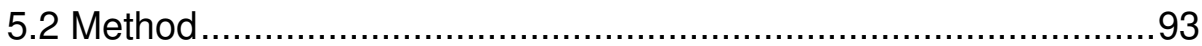

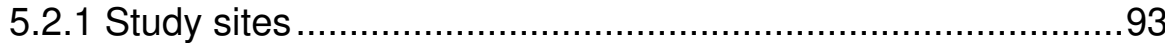

5.2.2 Experimental and measurement design ..............................94

5.2.3 Leaf level measurements ................................................95

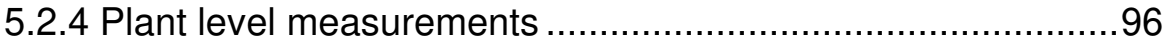

5.2.5 Community level measurements .........................................96

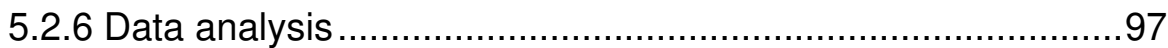

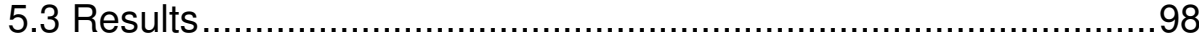

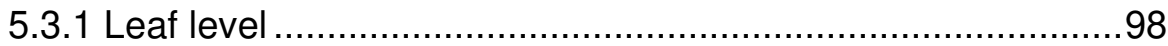




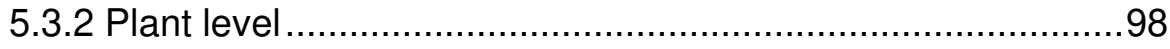

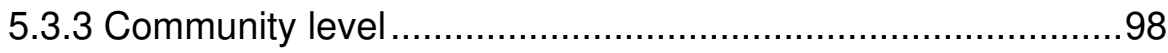

5.3.4 Biophysical properties ................................................100

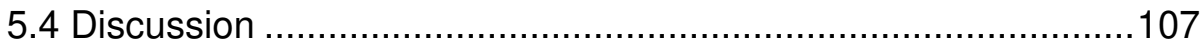

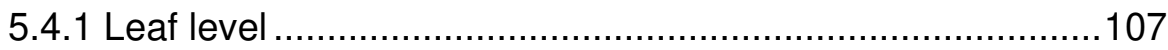

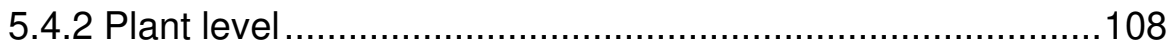

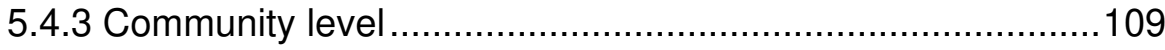

5.4.4 Recovery ................................................................. 110

5.4.5 Conclusion and application to other species ......................111

Chapter 6: Synthesis ...................................................................113

6.1 Vegetation structure as stress indicator..................................115

6.2 Physical stress indicators and corresponding vegetation indices 117

6.3 Resilience as stress indicator ..............................................120

6.4 Societal benefit and applications .........................................122

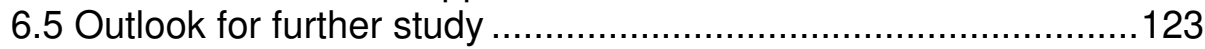

6.6 Conclusions and recommendations......................................125

Bibliography ...........................................................................127

Summary............................................................................................141

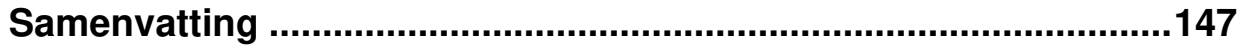




\section{Chapter 1: Introduction}

\subsection{Study scope}

The survival of humanity depends on the environment and the stability of the services that nature provides, such as food or oxygen. To quantify the importance of the benefits obtained from natural systems, the concept of ecosystem services was developed (Millennium Ecosystem Assessment 2005). Ecosystem services describe the benefits obtained from nature and can be used as a tool to estimate their monetary value (Costanza et al. 1998, Costanza 2000, Maes et al. 2012, 2016), thus highlighting their societal and economic relevance. Continued service provision is vital, and monitoring the stability of these ecosystem services should have a high priority. Ecosystem services are often provided by a combination of species. Establishing the contribution of a species to a specific service can be difficult. In some cases this might seem easy, such as for crops that produce food for cattle. However, these crop plants depend on other organisms to break down organic components into nutrients accessible to them. Moreover, often these plants will depend on yet other organisms to reduce pests. In a natural ecosystem any service is indirectly provided by many species. To monitor the ecosystem services provided, ideally that ecosystem should be monitored more broadly without focusing solely on the species providing the service. In this study we aim to establish how ecosystems can change, what the underlying drivers behind these changes are and finally how the stability of these systems can best be monitored. In this study we focus on coastal saltmarsh ecosystems, because (1) these systems provide many valuable ecosystem services (Boesch and Turner 1984, Deegan et al. 2002, Barbier et al. 2008, Koch et al. 2009, Morgan et al. 2009, Chmura 2011) and (2) monitoring these systems is difficult given their dynamic nature. Many of these ecosystem services are directly related to vegetation such as wave attenuation (Möller 2006, Koch et al. 2009, Morgan et al. 2009), or food provision (Rhee et al. 2009, Patel 2016). Therefore the health of the vegetation in this system is a large factor in determining the stability of the ecosystem services.

To study the stability of ecosystem services, we focus not only on catastrophic changes in an ecosystem such as a system changing from a vegetated to an unvegetated state (e.g. due to erosion), but also on smaller shifts like changes in the vegetation composition, as such smaller shifts can also be disruptive for an ecosystem service.

There are three major ways a system can change:

1) Sudden natural stochastic event (such as large scale flood deposited wrack, earthquakes etc); the occurrence of these events is often difficult to predict, as is the effect they are likely to have on vegetation. Although these 
events play a role in ecosystem service stability, they are not included in the monitoring of the stability of ecosystem services in this study.

2) Direct anthropogenic interference; the prediction of these occurrences and their effect on vegetation development is outside of the scope of this study, and is not considered further.

3) Stress induced changes in vegetation composition; for example an increase in nitrogen availability or the introduction of a new species into a system (invasive or natural succession) can change the competitive balance leading to a shift in species composition.

Theoretically it is possible to have a shift in vegetation composition without stressing the established vegetation, when rejuvenation of species is inhibited by competitors but adult specimens do not suffer stress from competition. Although it could be argued that established vegetation will be stressed prior to dying (of old age) before the shift in vegetation composition can occur. In this study we assume that prior to a change in vegetation composition, the system will be increasingly stressed.

Please note that we do not focus on natural succession, the natural processes where stronger vegetation types continuously take over (e.g. a grassland turns into a shrub system which turns into a forest). In coastal saltmarsh ecosystems, harsh conditions generally force a permanent pioneering stage and natural succession is regulated by stress. Often only when the abiotic environment changes, (e.g. elevation) will a high marsh species, which is generally the next natural succession stage, take over an area from a low marsh species. This will cause competition stress for both species, which causes 'stress induced changes in the vegetation pattern' (see: Bertness et al. 1992). We therefore focus on stress to measure ecosystem service stability.

\subsection{Vegetation stress}

We define stress similar to Lichtenthaler (Lichtenthaler 1996), where stress is defined as anything that negatively affects the growth and development of a plant. Lichtenthaler (1996) notes that not every deviation from the optimum immediately results in stress, and he therefore distinguished between eu-stress and di-stress. Eu-stress is a very mild deviation from the optimum that helps activate the plant to grow. Di-stress is clearly negative and has a negative effect on growth. We define stress similar to Lichtenthaler's definition of di-stress, i.e., we consider stress to mean anything that negatively affects plant development. With this definition, prior to a change in vegetation composition the declining species has to suffer from stress. This is a relatively broad definition, as any disturbance in the chemical processes that allow the plant to take in nutrients, water, light, carbon dioxide, etc can be a stressor. Generally, these stressors are addressed at a higher level, light 
limitation by other plants is called light competition. Nutrient limitation by other plants is called nutrient competition. Grime (Grime 1974, 1977) developed a system to group stressors, he argued that plants can specialize in 1) recovering from physical disturbance (e.g. grazing), 2) competition or 3) resisting stress. He argued that the extent to which a plant can invest in any one of these is limited and therefore a plant can never be good at all three. He proposed a triangle with these three aspects as axes, and argued that every plant has a niche within the triangle.

It is immediately clear that this does not match with our definition of stress. Following Lichtenthaler (1996) we also consider competition and physical disturbance as stressors. There are so many different aspects of stress interacting that it becomes highly complex to almost impossible to quantify individual aspects (e.g., a plant can be good at resisting nitrogen deficiency but suffer greatly when aluminum toxicity occurs). However, it is an interesting thought experiment, suppose instead of a triangle we use a multidimensional system where every potential stressor has an axis. In such a system the niche of every species would represent a multi-dimensional shape (the niche shape). The current conditions of any given habitat are a single point in this system (the habitat point), the further to the edge of the niche shape the habitat point moves, the more stressed the plant will become. We could even expect that the impact of a potential stressor is directly related with the width of the niche shape on that specific axis, i.e. a species with a very narrow range with regards to a single stressor will likely respond strongly when the habitat point moves over that axis. This thought experiment indicates the complexity of stress, as there are many potential stress axes for which each plant can have a different sensitivity. However, in practice, it will be difficult to determine the exact range and optimum for each salt marsh plant for every stressor, as well as the habitat conditions to establish the likelihood of a shift in vegetation type. Moreover, in this thought experiment we considered the effect of habitat conditions to be unidirectional, i.e. from the environment onto the organism. Thus, when the conditions change, the vegetation also changes. However, in real ecological systems this interaction is bidirectional, meaning that the habitat conditions are also affected by vegetation through so-called ecosystem engineering by plants (see: Jones et al. 2010). There are many examples of plants affecting their surroundings to exclude competitors (see: Goldberg 1990). This means that plants, although relatively limited, can influence the habitat point and pull it towards their niche shape. By doing this, they improve their own growing conditions. Often plants exchange one stressor for another, for which they have a higher tolerance. For example: they can decrease the $\mathrm{pH}$ to a level still tolerable by them, but no longer suitable for competitor's (See: Gagnon \& Glime 1992). Although a low pH decreases their growth as well, it reduces light limitation through competition. Thus by increasing stress for a factor for which they have a wide tolerance ('niche 
shape'), they can move towards more suitable conditions for an environmental factor for which they have a narrow tolerance (i.e. the habitat point moves closer to the center of the niche shape on the smaller axis), decreasing the overall stress. By reducing their own stress they can grow larger, remain healthier and thereby increase their ability to affect the habitat around them, creating a feedback.

\section{Stress-related transitions and positive feedbacks - requirements for monitoring}

Positive feedbacks can create alternate stable states by habitat modification, meaning that in one state the current conditions (the habitat point) are kept inside the tolerable range (the niche shape) by a specific plant species, while in another state the conditions move away from this species preferences, into the reach of another (plant) species who then pulls it further towards its preferred conditions (there are two niche shapes pulling on the habitat point). This is the basis for the alternate stable states theory introduced by (Pimm 1984) and applied more broadly by (Scheffer et al. 2001, 2009). This theory states that through feedbacks the environmental conditions can be kept stable for a specific organism/ecosystem beyond the point they might have otherwise shifted. A problem with these feedbacks is that while they are able to keep conditions stable up to a point, beyond this point a sudden decline can occur, creating a sudden large shift rather than a slow linear decline. To predict the distance to such a tipping point, normal stress indicators typically do not work (van Nes and Scheffer 2007, Kéfi et al. 2013). As alternative, critical slowing down has been proposed as suitable indicator (van Nes and Scheffer 2007, Kéfi et al. 2013). Critical slowing down means that when a system is close to a tipping point, the systems resilience is low, and hence it will take longer to recover from disturbance.

The existence of feedbacks and alternative stable states complicates our argument that stress is of vital import to ecosystems and should be monitored. Although stress decreases resilience, its effects might be difficult to distinguish until a sudden shift occurs. We therefore distinguish between linear and non-linear transitions. In a linear process, such as competition, the stronger competitor species slowly increases its cover and pushes the other species out. The progress of this can be observed with phytosociology (the study of plant communities). We call this linear because the chance a species disappears is directly related to its cover, i.e. a species with high cover is unlikely to disappear. Note that this may seem more complicated in terrestrial ecosystem than coastal systems, as in terrestrial systems many different species can live mixed together. Nevertheless, even in these terrestrial systems competition is mostly a linear process. Alternatively, a transition can be non-linear. In such a case a feedback stabilizes the system, reducing the visible effect of a stressor up to the tipping point. In this 
case phytosociology is unlikely to yield useful monitoring data. Therefore, depending on the strength of feedbacks keeping environmental conditions in place, the transition type will differ, and our monitoring techniques should reflect this.

\section{In situ monitoring: species composition, vegetation development, vegetation structure}

There is a long tradition of monitoring linear transitions in vegetation composition using phytosociology, the study where vegetation cover per species is used to monitor vegetation development. The fundaments for modern phytosociology were developed over a hundred years ago (see review: Westhoff 1967). This is still widely used to study a wide variety of ecosystem and monitor developments therein, several of our study sites in The Netherlands are monitored using this technique (Tolman and Pranger 2012, Paree 2017). A problem with this type of monitoring is the large temporal delay, a change is only detected after the vegetation type has shifted. The temporal delay is increased by the large workload of collecting and processing the data.

In addition to cover, vegetation height is often used to monitor vegetation development (as introduced by: Barkman 1979). Together with cover this is an approximation of vegetation volume. This gives additional insight as stressed vegetation might retain its cover but lose volume. Additionally, biomass is an often recorded measure, although this is applied most in research and agriculture. In agriculture, biomass production is one of the most important success indicators, as often product are sold per weight unit. Biomass gives even more information than the combination of height and volume, and although more labor intensive, much additional information can be obtained by further analyzing these harvested samples, by determining the water, pigment, nitrogen or phosphate content.

The previous measures deal with how much vegetation there is, but the spatial distribution of stems and leaves is not measured, although some indication of this vegetation structure can be derived from the standard deviation of vegetation height. Vegetation structure has since long been recognized in vegetation classification (Westhoff 1967), and is known to have large ramifications for ecosystem functioning (Wallis de Vries and Van Swaay 2006). However, a good way to quickly and objectively quantify vegetation structure is not yet available.

Non-linear transitions, transitions in feedback based situations, do not have a strong monitoring tradition. Recent studies have shown that recovery rate can be used to approximate the distance to a tipping point, and recovery rate has also been argued to be useful as a general stress indicator (Kéfi et al. 2013). A recent study showed that recovery rate can be used as indicator for tipping points in salt marshes (van Belzen et al. 2017). 


\subsection{Remote sensing monitoring}

In 1972, the first Landsat satellite was launched, making satellite data widely available. Since then remote sensing has greatly improved in resolution, both spatially and temporally. A spatial resolution less than a meter is possible, rivalling the spatial resolution of aerial photographs. The increase in both commercial and governmental satellites has greatly increased the temporal availability. It is therefore no surprise that satellite remote sensing data are increasingly being used to map and monitor a wide variety of ecosystem aspects.

One important way remote sensing is used, is for land cover identification. In these studies one or several ecotypes or vegetation types are distinguished, and consequently their development can be tracked through time (see review by: Kerr \& Ostrovsky 2003). In forestry satellite remote sensing is also used for estimating above ground biomass, the dead wood component of this biomass, the vegetation cover and species composition (Camarretta et al. 2020). In grassland and heathland systems remote sensing is used in a wide variety of ways, satellite data can be used to monitor plant biodiversity (Wang and Gamon 2019), assess conservation status (Schmidt et al. 2017, 2018), biomass (Guerini Filho et al. 2020), and chlorophyll content (Homolova et al. 2013, Tong and He 2017). Remote sensing is increasingly important for precision agriculture where it is used to analyze a variety of aspects such as soil conditions, pigments or biomass (see review by: Mulla 2013), for a complete review on grassland monitoring using remote sensing see also Ali et al. (2016). A recent review also demonstrated the value of remote sensing for examining the extend of the damage of detrimental stochastic events such a fire or an oil spill, and the consequent recovery (Reif and Theel 2017).

Few studies addressed stress in saltmarsh vegetation using remote sensing. Remote sensing was used to detect the stress and recovery of vegetation in response to a large oil spill in saltmarshes in Louisiana (Khanna et al., 2013). More generically, several studies focused on distinguishing between different salt marsh species and their development (Silvestri et al. 2003, Lee et al. 2012, Ouyang et al. 2013, Hladik and Alber 2014, van Beijma et al. 2014, Uossef Gomrokchi et al. 2020), and on estimating biomass (see review: Klemas 2013). Studies have used remote sensing to establish elevation and construct a Digital Elevation Model (e.g.: Hladik \& Alber 2012; McClure et al. 2016; Rogers et al. 2018), including vegetation height (Collin et al. 2018) and DEMs have been used to map channels (Zheng et al. 2016).

It has even been found possible to model soil properties (salinity and water content) of salt marshes (Zhang et al. 2019). It seems likely that vegetation monitoring 
techniques that were developed for terrestrial ecosystems can be adapted for use in salt marshes. However, before applying these techniques to salt marshes, it has to be established which vegetation properties relate well with stress in salt marshes.

\section{Different levels to measure stress: from leaf to community level}

When measuring vegetation properties in general, and stress in particular, there are different potential measurement levels. Vegetation stress can be examined at cellular level, leaf level, plant level, community level or (meta-) population level. We currently do not know how these different levels relate to each other. Although it seems obvious that a community of stressed plants will itself show signs of stress, this does not necessarily have to be the case.

In this study, we consider monitoring at the leaf, plant and community level. Most established techniques for measuring vegetation development focus on either the plant or the community level. From a practical perspective, community level monitoring seems to make most sense, as this type of data can be efficiently collected, and could potentially also be derived using satellite and airborne sensors. Leaf and plant level data have to be collected in situ and will likely require more additional processing. However, it remains unclear what measurement level would be most appropriate to analyze vegetation stress and stability.

\section{Underlying drivers}

Stress indicators can only indicate the current stress level. Additional analyses may be necessary to establish what possible mitigating measures could be required to reduce the stress levels. In some cases it is obvious what the stressor is, e.g. an invasive species is outcompeting native vegetation. But in most cases there is a complex combination of intermixed stressors present. Understanding what processes drive the development of the landscape, and how the major stressors affect the vegetation is however of vital import to take effective action to protect an ecosystem service. 
Figure 1.1 Schematic overview of measurement levels addressed in chapters two, three, four and five.

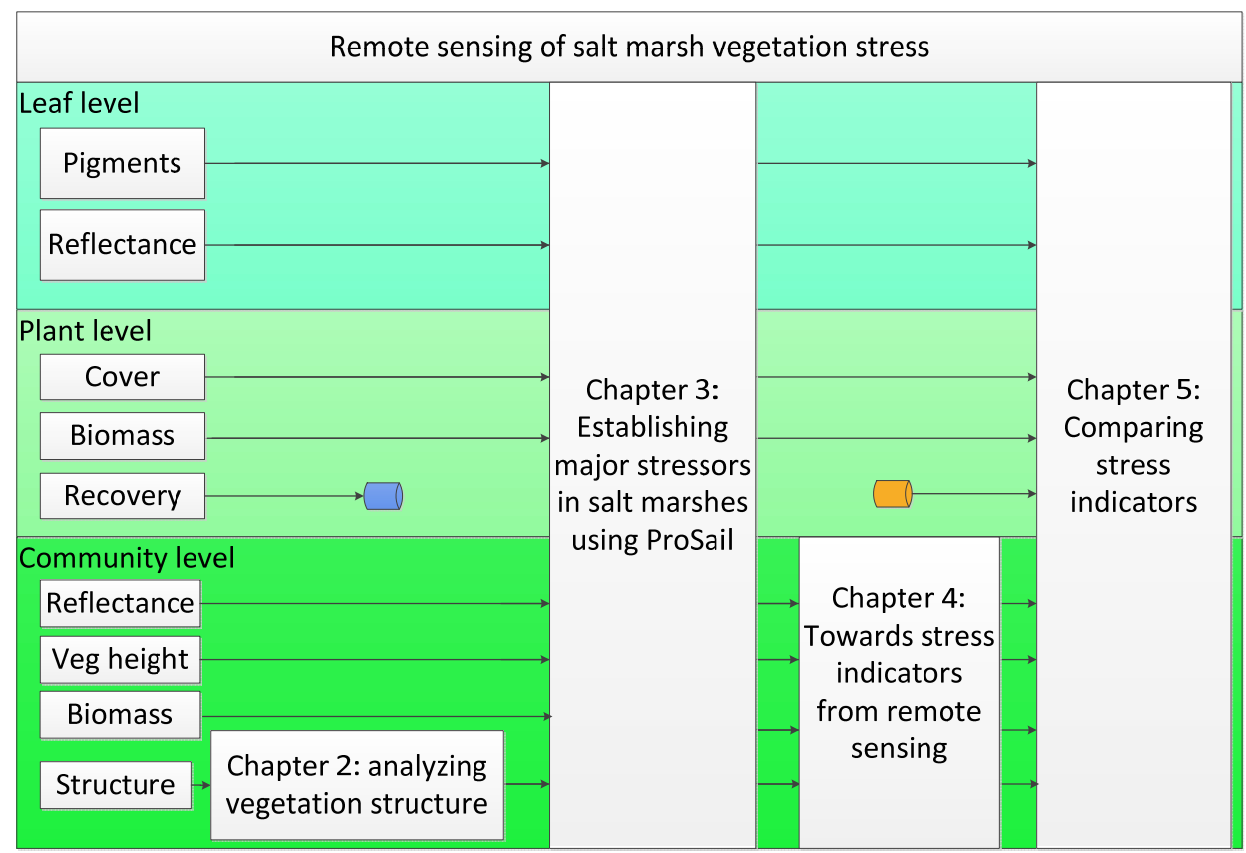




\subsection{Aim and objectives of this thesis}

The aim of this study is to establish efficient stress indicators to monitor ecosystem service stability in European coastal marshes, to help safeguard vital ecosystem services. We assume any drastic decrease in ecosystem service provision will be preceded by vegetation stress, hence, we look into vegetation stress indicators. Plant structure is a potentially vital component of vegetation stress, but cannot currently be quantified easily in situ. Therefore in our second chapter we address the research question:

1) How to include high-detail in situ structure measurements in our stress indicator assessment?

As we aim towards deriving stress from remote sensing data, we will require insight into how vegetation properties are affected by major stressors, and how these in turn affect vegetation reflectance. In our third chapter we therefore look into our second research question:

2) How do the major stressors in salt marshes, affect vegetation properties and how do these properties affect reflectance?

To assess the potential of satellite remote sensing data, we use satellite data to analyze a relatively simple and monospecific system (reed lands) that provide many vital ecosystem services on a large spatial scale (the Danube delta). In the fourth chapter the third question is addressed:

3) Can we use satellite remote sensing data to establish an indicator for reed development?

Then we assess what suitable indicators for salt marshes might be, and how these represent different types of stress, when we address the fourth research question, in chapter five:

4) How well do potential stress indicators, including recovery rate, represent environmental and competition stress?

Finally, in chapter six (the synthesis) we combine the previous chapters and discuss how this all fits together, and we present what we think are promising starting points for continued research. Figure 1.1 schematically shows which measurement levels are addressed in each chapter. 


\title{
Chapter 2: Depth from focus with a regular camera to analyze small scale habitat structure
}

\author{
B. Oteman ${ }^{1 *}$, S. Nieuwhof ${ }^{1}$, T.J. Bouma ${ }^{1}$, D. van der Wal ${ }^{1,2}$
} ${ }^{1}$ NIOZ Royal Netherlands Institute for Sea Research, Department of Estuarine and Delta Systems, and Utrecht University, P.O. Box 140, 4400 AC Yerseke, the Netherlands.

${ }^{2}$ Faculty of Geo-Information Science and Earth Observation (ITC), University of Twente, P.O. Box 217, 7500 AE Enschede, The Netherlands.

* Author to whom correspondence should be addressed: bas.oteman@nioz.nl

\section{Abstract}

Habitat structure is important in many aspects of ecology. Many species, trophic interactions and ecosystem services depend on habitat structure. In agriculture vegetation structure is recognized as a valuable predictor for crop yield. However describing vegetation structure in three dimensions remains difficult. We propose a new method of analyzing habitat structure: Depth From Focus. By modifying the software on a DSLR camera it can be used to take pictures of a single location with slightly different points of focus. These pictures can then be transformed into a $3 d$ representation. We validated this technique by applying it to controlled and field situations, and at different spatial scales (grass seeds and patches of vegetation). Extensive validation showed Depth from focus performs well, both under controlled circumstances and in the field and on different scales. We could accurately represent grass seeds and vegetation structure and could differentiate between various vegetation types.

Depth from focus provides an easy to use tool for studying small scale structures. It does not require expensive specialized equipment, it can be applied to different scales and it performs well. The two main drawbacks are 1) that the subject should not move while pictures are being taken for an amount of time depending on desired depth resolution, and 2) that this method does not offer a full $3 d$ result, as it is currently only calibrated for a single point of view. When these are taken into account depth from focus can be used to map habitat structure on various scales with high spatial resolution, and although applying it to a specific set of circumstances will require additional calibration, this method looks promising for many areas of study as a cheap and quick method of measuring habitat structure.

Keywords: depth from focus, focus stacking, vegetation structure, habitat structure, DSLR camera 


\subsection{Introduction}

Habitat structure has been called 'a key driver of ecological function' (Shugart et al. 2010, Anderson et al. 2015). Many ecosystem services such as flood protection (Costanza et al. 1998, Möller 2006), wave attenuation (Bouma et al. 2005) and recovery after drought (Costanza et al. 1998), are a direct result of habitat structure. Other services indirectly depend on habitat structure. For example structure affects pollination by bees (Cho et al. 2017) and it can modulate biological control (Obermaier et al. 2008). Vegetation structure has also been shown to be of great importance for many species such as grasshoppers (Joern 1982), ground beetles (Brose 2003), spiders (Gunnarsson 1990) and lizards (Martín and López 1998). In agriculture, structure has been recognized as a valuable predictor for crop yield. Plant structure and architecture is crucial for understanding the resource capture strategies and adaptations to climate (Valladares and Niinemets 2007, Nock et al. 2013). Vegetation canopies and their structure are also used for stocking calculations, i.e. to derive the amount of livestock an area can support (Harmoney et al. 1997). In crop breeding it is used to describe crop phenotypes, to select genotypes for optimal yield ( $\mathrm{Li}$ et al. 2014). As a consequence, food production, perhaps the most important ecosystem service to humanity (Costanza et al. 1998), may be estimated from habitat structure.

Despite its widely recognized importance, quantifying habitat structure is highly complicated (Harrell and Fuhlendorf 2002, Kazmi et al. 2014, Schima et al. 2016). Measuring in two, or even three dimensions is a source of inaccuracy. In addition interpretation of data can be difficult (Harrell and Fuhlendorf 2002, Kazmi et al. 2014). Here we propose a new technique, along with some descriptors of structure, to quantify the small-scale habitat structure of grassland vegetation.

Vegetation structure can be measured with various methods (table 2.1), such as the Portable Photo Frame (PPF) method, Terrestrial Laser Scanning (TLS), Time of Flight (ToF) photography, Stereo Vision (SV) and Structure From Motion (SFM). The Portable Photo Frame (PPF) method is an adaptation of vegetation profile boards (Guthery et al. 1981) where a red board is placed in the vegetation, which can easily be separated from the green vegetation (Möller 2006, Rupprecht et al. 2015). Using this method the structure description is obtained locally along a narrow width line transect, and it is limited to relatively low vegetation, such as grasslands. The resolution depends on the camera used and the size of the profile board. This method is not completely non-destructive, as vegetation may not obstruct the line of sight from the camera to the vegetation under investigation. PPF generally performs well in creating a side looking structure profile of a limited range of vegetation types. 
Terrestrial Laser Scanners (TLS) are expensive and often not practical to use in vegetation (Schima et al. 2016). In addition they have to be several meters away from their target to get a valuable point cloud (Nock et al. 2013), making them more applicable for large scale structures.

Time of Flight cameras (ToF) are the only source that offer instant 3d images without further processing. ToF cameras have been used successfully to analyze vegetation properties (Busemeyer et al. 2013). The performance of ToF may however suffer from sunlight, as it is hard to differentiate between reflected and naturally occurring light. This makes deploying them in the field difficult ( $\mathrm{Li}$ et al. 2014). Under natural lighting conditions, calibration may be needed to account for absorption and scattering of light by the leaves (Kazmi et al. 2014). The resolution of ToF cameras is generally low, especially when compared with normal cameras. Stereo Vision (SV) and Structure From Motion (SFM) depend on overlap between images to calculate angles between objects and the camera to estimate distance. SV uses multiple cameras, SFM uses multiple images by one camera. Stereo Vision can be used very quickly in the field, although significant processing afterwards is required (Kazmi et al. 2014). The resolution of SV and SFM depends on the camera used. SFM is the only method that offers a complete $3 d$ representation of an object, as it depends on using multiple points of view and uses multiple images to fill in blank spots that cannot be seen from one viewpoint. All other systems can only detect a single side of an object. These techniques require an unobstructed line of sight, and can therefore never see all sides of an object. It is possible to place reference markers to combine multiple points of view, this is often done in TLS, but this could be done in other techniques as well.

The performance of both SV and SFM decreases when applied to vegetation in a field situation, as it depends on being able to identify matching features, which can be difficult in vegetation as this often has a homogeneous structure and lacks surface texture (Westoby et al. 2012, Li et al. 2014). Both techniques can also be compromised by changes in light condition (Kazmi et al. 2014, Li et al. 2014).

A new passive technique is the use of a light-field camera (Georgiev et al. 2013). These cameras can instantly record light intensity and angle, allowing them to instantly create $3 d$ images (Schima et al. 2016). However, recording these angles reduces the sensors available to measure light intensity, drastically reducing the resolution (Kazmi et al. 2014). The distance estimates by the light-field camera can also incorporate a significant error, especially at larger distances (Schima et al. 2016).

Here, we propose an alternative method that allows capturing $3 d$ images in the field quickly, using a simple Digital Single Lens Reflex (DSLR) camera, without sacrificing image resolution. This method, 'depth from focus', was originally introduced by Grossmann (1987) and a simplified version is currently in use in 
regular photography, where it is known as 'focus stacking'. Focus stacking combines multiple pictures with different focus depths into one sharper image, effectively increasing the depth of field (the in-focus area) of the image (Jacobs et al. 2012). Depth from focus is currently used in microscopy, where differently focused images are used to create detailed images of, for example, blood cells (Gorthi and Schonbrun 2012). The use of this technique to study habitat structure in grasslands is new. This approach provides a multitude of advantages: it only requires a simple digital camera and a tripod, it is cost efficient and the level of detail is limited by the camera resolution only. DFF does not have requirements with regard to leaf structure, nor does it require recognizable features. It only requires small scale differences in texture, which are generally available in natural situations. The method can be applied at different scales, at different orientations, and it can be applied to any natural object.

The aim of this paper is to proof the concept of depth from focus as a new technique for quantifying habitat structure of vegetation, and explore its potential and limitations. We will first introduce the method technically, followed by performance validations. Subsequently we show how this method can be applied to micro habitat analyses and how it might be used to quantify the difference in vegetation structure of salt marsh species. 
Chapter 2: Depth from focus with a regular camera to analyze small scale habitat structure

Table 2.1. Summary of the various methods to measure structural complexity.

\begin{tabular}{|c|c|c|c|c|c|c|c|}
\hline $\begin{array}{l}\text { Technique } \\
\text { name }\end{array}$ & Resolution & $\begin{array}{l}\text { Collection } \\
\text { speed }\end{array}$ & $\begin{array}{l}\text { Processing } \\
\text { required }\end{array}$ & 3d type & $\begin{array}{l}\text { Applicability } \\
\text { vegetation }\end{array}$ & $\begin{array}{l}\text { Cost } \\
(€)\end{array}$ & Source \\
\hline $\begin{array}{l}\text { Terrestrial } \\
\text { Laser } \\
\text { scanner }\end{array}$ & Variable & 5 min & Yes & Envelope & Average & $>15000$ & $\begin{array}{l}\text { (Nock et al. } \\
2013 \text {. Schima } \\
\text { et al. 2016) }\end{array}$ \\
\hline Time of flight & $0.04 \mathrm{MP}$ & Instant & No & Envelope & Poor & $\begin{array}{l}200- \\
500\end{array}$ & $\begin{array}{l}\text { (Kazmi et al. } \\
2014)\end{array}$ \\
\hline Stereo vision & Variable & Instant & Yes & Partial & Poor & $\begin{array}{l}200- \\
500\end{array}$ & $\begin{array}{l}\text { (Kazmi et al. } \\
2014 \text { ) }\end{array}$ \\
\hline Light-field & $1 \mathrm{MP}$ & Instant & Yes & Envelope & Good & 100 & $\begin{array}{l}\text { (Schima et al. } \\
2016 \text { ) }\end{array}$ \\
\hline $\begin{array}{l}\text { Structure } \\
\text { From } \\
\text { Motion }\end{array}$ & Variable & 5 min & Yes & Full 3d & Poor & 150 & $\begin{array}{l}\text { (Westoby et } \\
\text { al. 2012b) }\end{array}$ \\
\hline $\begin{array}{l}\text { Depth From } \\
\text { Focus }\end{array}$ & $18.9 \mathrm{MP}$ & $5 \mathrm{~min}$ & Yes & Envelope & Good & $\begin{array}{l}200- \\
500\end{array}$ & This paper \\
\hline $\begin{array}{l}\text { Portable } \\
\text { Photo Frame }\end{array}$ & Average & $5 \mathrm{~min}$ & Yes & $\begin{array}{l}\text { Fixed } \\
\text { size } \\
\text { envelope }\end{array}$ & $\begin{array}{l}\text { Good, } \\
\text { only side }\end{array}$ & $\begin{array}{l}200- \\
300\end{array}$ & $\begin{array}{l}\text { (Möler 2006, } \\
\text { Rupprecht et } \\
\text { al. 2015) }\end{array}$ \\
\hline
\end{tabular}

\subsection{Materials and methods}

\subsubsection{Focus stacking theory}

Focus stacking uses a series of photos taken at different focus distances. From each picture, the in focus pixels are selected and together they are used to create a fully in focus image. Edge detection algorithms can be used to select in focus pixels. If an object in an image is out of focus, it is blurred, i.e., the variation with neighboring pixels in an image is low, whereas if an object is in focus, the edges are sharp and the textural variation is high. This implies that areas should have sufficient contrast to ensure enough variation. While focus stacking combines images into a sharper picture, depth from focus uses the position at which each pixel was found sharpest, and uses that to create a 'depth' map. The distance corresponding to a position in the image stack is lens dependent and can be derived by using information provided by the lens manufacturer.

\subsubsection{Camera setup and stack preprocessing}

We used a Canon 600D DSLR camera with a common 18-55mm f/3.5-5.6 IS STM lens. Alternative firmware (Magic Lantern, firmware version 1.0.2 (3.8.3), nightly build of 3-1-2015) allowed us to take focus stacks up to 100 images at a time. A 
$10 \mathrm{~ms}$ delay between pictures was used, with a start delay of 1 second and the 'step wait' option was enabled (only takes picture after refocusing is complete). The stacks of images were processed with the professional version of Zerene Stacker (version 1.04 build 201412212230), following Brecko et al. (2014). This tool automatically handles rescaling of the images, and it evaluates contrast in a small neighborhood around each pixel position to estimate focus. Next the program applied a weighted interpolation between images on the estimated focus to calculate a depth for every pixel. Finally the dmap tool of Zerene Stacker was used to remove all out of focus pixels from each image. By combining these images, we know for each pixel in which image (indicative for focus distance) it was sharpest. The distance estimation allows us to map the total number of pixels at each distance, however a pixel focused on a faraway object represents a larger surface area than a nearby pixel. In the appendix a formula is provided to correct for this effect.

\subsubsection{Lens calibration and focus distance estimation}

When changing focus distance, the lens changes position in relation to the sensor. The distance from the lens to the sensor is inversely related to the distance from the object to the lens.

This relation is described by the thin lens equation:

$$
\text { 1. } \frac{1}{f}=\frac{1}{-S_{x}}+\frac{1}{S_{y}}
$$

Where $f$ is the focal length, which is a property of the lens. The focal length is generally written on the exterior of the lens, and stored in the image metadata. In our case the lens is capable of $f$ values between $18 \mathrm{~mm}$ and $55 \mathrm{~mm}$, but was fixed at $18 \mathrm{~mm} . S_{x}$ is the distance from the front nodal point of the lens to the object being photographed. $S_{y}$ is the distance from the sensor to the rear nodal point. This formula can be rewritten as:

$$
\text { 2. } S_{y}=\frac{-f S_{x}}{-S_{x}+f}+C
$$

It should be noted that often the thin lens equation does not reliably model the thick lenses used in DSLR cameras, especially at smaller focus distances. Therefore an offset value, $\mathrm{C}$, was introduced that accounts for the distance offset between the sensor and the studied object. To derive the distance between the studied object and the lens a calibration stack was made. The calibration stack was used to determine the step size i.e. the smallest possible change in the distance between 
the lens and the sensor, and to determine the total range of this distance. This range is determined by the maximum focus distance (when the focusing distance goes to infinity) and the minimum focusing distance, which was derived using the calibration stack. To construct the calibration series, a tape measure was placed on a table, the front of the camera was aligned with the 0 marker. Three stacks were averaged to establish the calibration curve, allowing the distance to be estimated from each picture. This yields the distance of each focus step to the front of the camera. Afterwards focus range, minimum focus distance and distance offset were derived by fitting equation 2 to the calibration curve using non-linear least squares. To calculate the focus distances for a different lens, only the focal length (f) is required, the same method will apply. It was noticed that, when fitting the function for new lenses, the fit is sometimes drastically improved when the last few points, where the lens focal points tend to infinity, are ignored. In our case removing only the last point before the lens reached infinity was sufficient.

The number of focus steps is lens dependent, and some lenses require more than 100 steps to cover the entire focus range. However, the default version of Magic Lantern is limited to a maximum of 100 steps. To overcome this problem we adapted the source code and raised the limit to 1000 steps.

When focusing, the camera makes an estimate of the focus distance internally, which is stored in the image metadata (exif), which was extracted with the ExifTool 10.43 (Harvey 2007). From the additional information obtained from exif, the upper and lower bounds of the focus distance estimate were averaged and compared to the calculated values. In addition, the distance from the front of the lens to the object was measured manually. The distance estimates from the exif and the manually obtained distances were aligned based on the first value (step 0) and compared.

Depth from focus has to be calibrated for each lens, to be able to translate from position in the stack to distance. To test how well the technique performs with a completely different lens, we also applied the technique to a macro scale. A Sigma $105 \mathrm{~mm}$ macro lens was used, and calibrated as described before. This lens has an $f$ (aperture) of 2.8 and a minimal focus distance of $31.2 \mathrm{~cm}$ from the sensor. This lens did not store distance estimates in the exif, hence it was calibrated manually. 


\subsubsection{Validation of distance estimation in the laboratory}

To test the accuracy of the distance estimation, we took samples of an object of known height in controlled circumstances. Indoor, we covered the ground surface with textured paper on which we placed textured objects $(4 \times 4 \mathrm{~cm}$, height: $7 \mathrm{~cm})$. The camera was placed at approximately one meter height, facing down. A stack of 156 images was created. Once this was completed a textured object was placed on top of the previous one and a new image stack was made. This procedure was repeated until a height of $56 \mathrm{~cm}(8 \times 7)$ was reached. These eight stacks were processed to depth maps. In each depth map the difference between background and textured objects was extracted by averaging both surfaces using the image classification tool in ArcGIS 10.1. The differences between these surfaces are compared with the known height of the textured objects. The increment between each consecutive stack was calculated and compared with the known value $(7 \mathrm{~cm})$. This procedure was repeated 3 times, resulting in $24(3 \times 8)$ image stacks. These stacks were processed twice; once with the empirically derived distance profile and once with the distance profile provided by the lens (in the exif data).

\subsubsection{Validation of seed thickness in the laboratory}

A manual calibrations was used to estimate thickness of common cord grass seeds (Spartina angelica), applying the macro-lens as described above. In the laboratory, 18 seeds were measured manually using a caliper and with the depth from focus technique (see fig 3). A seed was placed on millimeter paper, the front of the camera was mounted $10 \mathrm{~cm}$ above the seed and stacks of 51 images were made. In ArcGIS the resulting depth maps were sampled manually at the middle of the seed, at the same position as we measured with the caliper, and at the ground surface next to the seed. The thickness of the seed is the difference between the two measurements.

\subsubsection{Validation of saltmarsh vegetation structure in the}

\section{laboratory}

To validate how well depth from focus represents vegetation structure we manually measured vegetation structure under laboratory conditions. We collected a patch of $20 \times 20 \mathrm{~cm}$ of Spartina vegetation, and transported it with about $30 \mathrm{~cm}$ of soil to the laboratory. We took a focus stack at nadir, at around $20 \mathrm{~cm}$ above the highest point of the plants. The stack was processed using the best performing depth profile (the empirical depth profile). We then placed a ruler next to the camera and 
aligned the zero with the front of the camera lens. We then placed two laser levels on tripods next to the plants, made sure they were level and aligned them with a specific distance on the ruler. Without having moved the camera after taking the focus stack, we took a single picture. This was repeated for every centimeter between 62 and 20 centimeter from the camera, covering the entire range of vegetation height. This resulted in a set of images that were aligned with the focus stack, and where vegetation of a specific height was colored red by a laser. To select the laser-affected pixels in these images, we used a threshold on the brightness in the red band of 250 . To avoid naturally occurring red we required brightness in the green band to be $>230$, this worked because our laser mostly saturated the camera pixels in all bands (producing white). To not exclude naturally occurring brown areas, brightness in the green band was also allowed to be $<120$ when brightness in the red was $>250$. Pixels matching the criteria of being laseraffected were clustered. Clusters were made by checking if direct neighbors $(8 \mathrm{x})$ were also affected. The laser light created relatively large solid clusters, hence we did not have to correct for many small fragmented clusters close together. The clusters were loaded into ArcGIS. To ensure we measured the correct location, the position of every cluster was manually checked and if necessary rectified using the georeferenced tool in ArcGIS. To avoid pseudo replication we extracted the average depth for each cluster from the depth map produced by the focus stack using the zonal statistics tool. This allowed us to compare the depth estimated by depth from focus with the distance known from the laser measurement in a vegetation setting.

\subsubsection{Application to saltmarsh vegetation structure in the field}

Using depth from focus we investigate how the vegetation structure depends on vegetation type in grasslands. Salt marshes offer a convenient study area as they offer several contrasting grassland vegetation structures, and in these ecosystems vegetation structure directly provides an important ecosystem service, i.e. wave mitigation (Möller et al. 2014, Rupprecht et al. 2015). This becomes increasingly important in face of sea level rise and additional stress on coastal defenses.

The data were collected in May 2015 in a saltmarsh named 'Paulina' along the Dutch Westerschelde estuary, located at $51.35^{\circ}$ latitude and $3.718^{\circ}$ longitude. The saltmarsh consisted mainly of the pioneer species common cord grass (Spartina anglica), a standing grass that can cope with regular flooding. In addition, sea couch grass (Elytrigia atherica), which is a laying grass, and sea purslane (Atrixplex portulocoides), a small shrub species, were present. In total 26 plots were measured (10x Spartina, 8x Elytrigia, 8x Atriplex). In the center of each plot 
an image stack of 51 images with a step size of 2 (skipping one focus step after each image), was taken at nadir. The front of the camera was placed approximately $15 \mathrm{~cm}$ above the highest point of the vegetation.

The depth maps derived from the stacks were described using several statistical measures of structure, following Bretar et al. (2013) for the first three and adding Moran's I (Moran 1950) as another often used tool to describe spatial structure:

- Vertical roughness, expressed as the root-mean-square of the estimated distance.

- Spatial autocorrelation or correlation length, defined as the length where the autocorrelation function is equal to $1 / \mathrm{e}$.

- Tortuosity, calculated using the 'computeAreaRaster' tool from the movement-based kernel density estimates (MKDE) package in $r$. The method of calculation was similar to Bretar et al. (2013).

- Moran's I, using a window size of 101 pixels. The window size for the Moran-I analysis is very important, but can get very computationally expensive in these high resolution images, as this window is calculated for each pixel.

These four structure parameters were compared between the three vegetation types using an ANOVA and Fisher's least significant difference (LSD) post hoc test.

\subsection{Results}

\subsubsection{Validation of distance estimation in the laboratory}

The relation between focus step and distance shows an asymptotic curve, as expected. They also show a high similarity between empirical and automatic distance profile (figure 2.1). This clearly shows that by fitting a function through the automatic distance estimates, these estimates can be used for depth from focus without a lengthy and involved calibration process for every lens. Despite the high similarity between automatic and empirical calibration, the performance analysis shows that the empirical calibration outperforms the automatic calibration (figure 2.2). The empirical profile showed an average increase of $7.485 \pm 1.12 \mathrm{~cm}$, where the actual increase was $7 \mathrm{~cm}$. The lens based profile had an average of $6.20 \pm 1.56$. This shows both perform well, but the empirical calibration performs better, likely because the automatic calibration data is binned by the camera (figure 2.1). 


\subsubsection{Validation of seed thickness in the laboratory}

We applied the same technique to another lens, and measured grass seeds using a caliper and using depth from focus. We found a good agreement between the two, with an $r^{2}$ adj of 0.89 . There is a slight overestimation by the camera (figure 2.3).

\subsubsection{Validation of saltmarsh vegetation structure in the}

\section{laboratory}

To evaluate the performance in vegetation like structures we compared vegetation height measurements using a laser system with depth from focus height estimates. This showed a good agreement $\left(r^{2} a d j=0.903\right)$ (see figure 2.4).

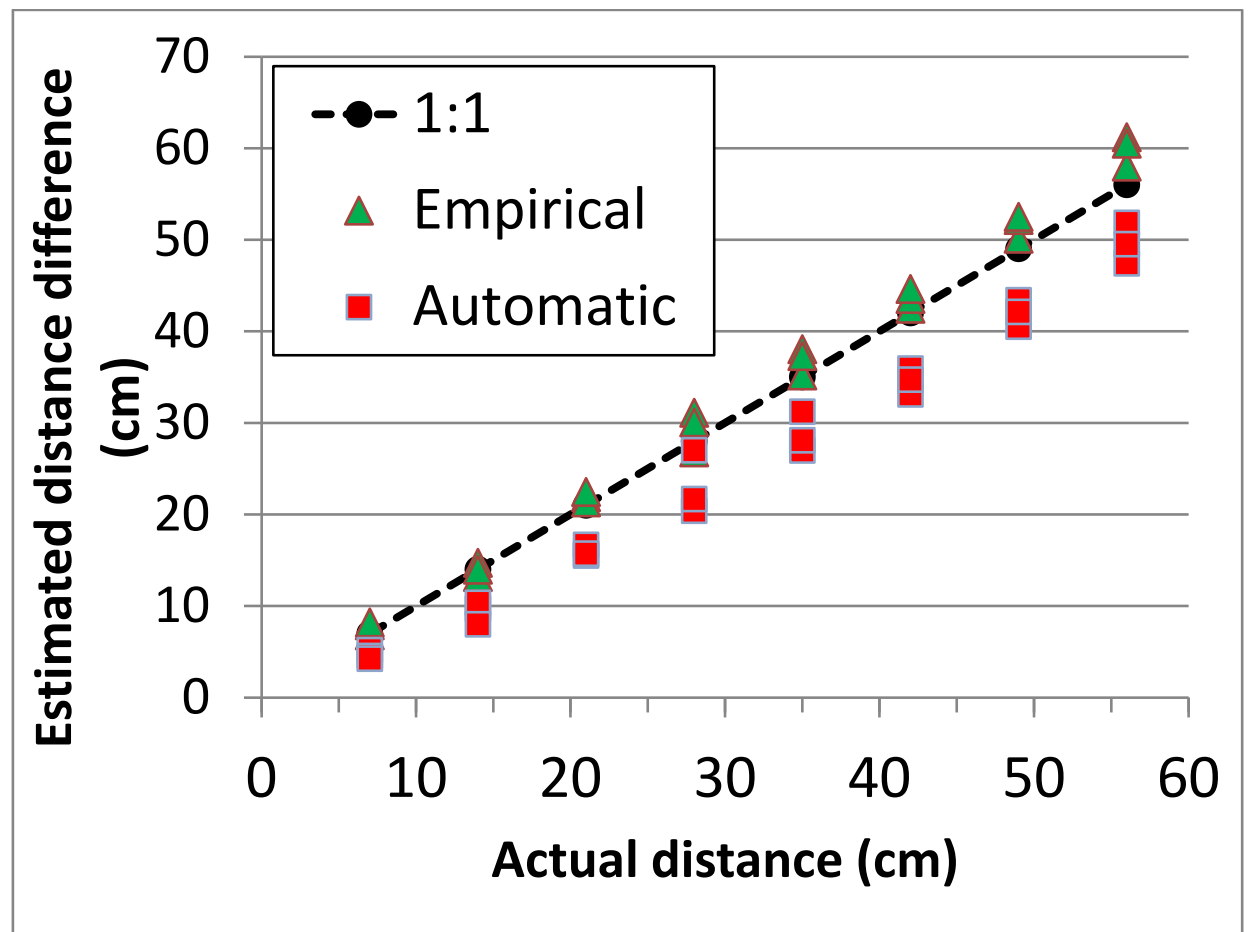

Figure 2.1. The relation between focus step and distance, empirically established and automatically estimated by the camera. 


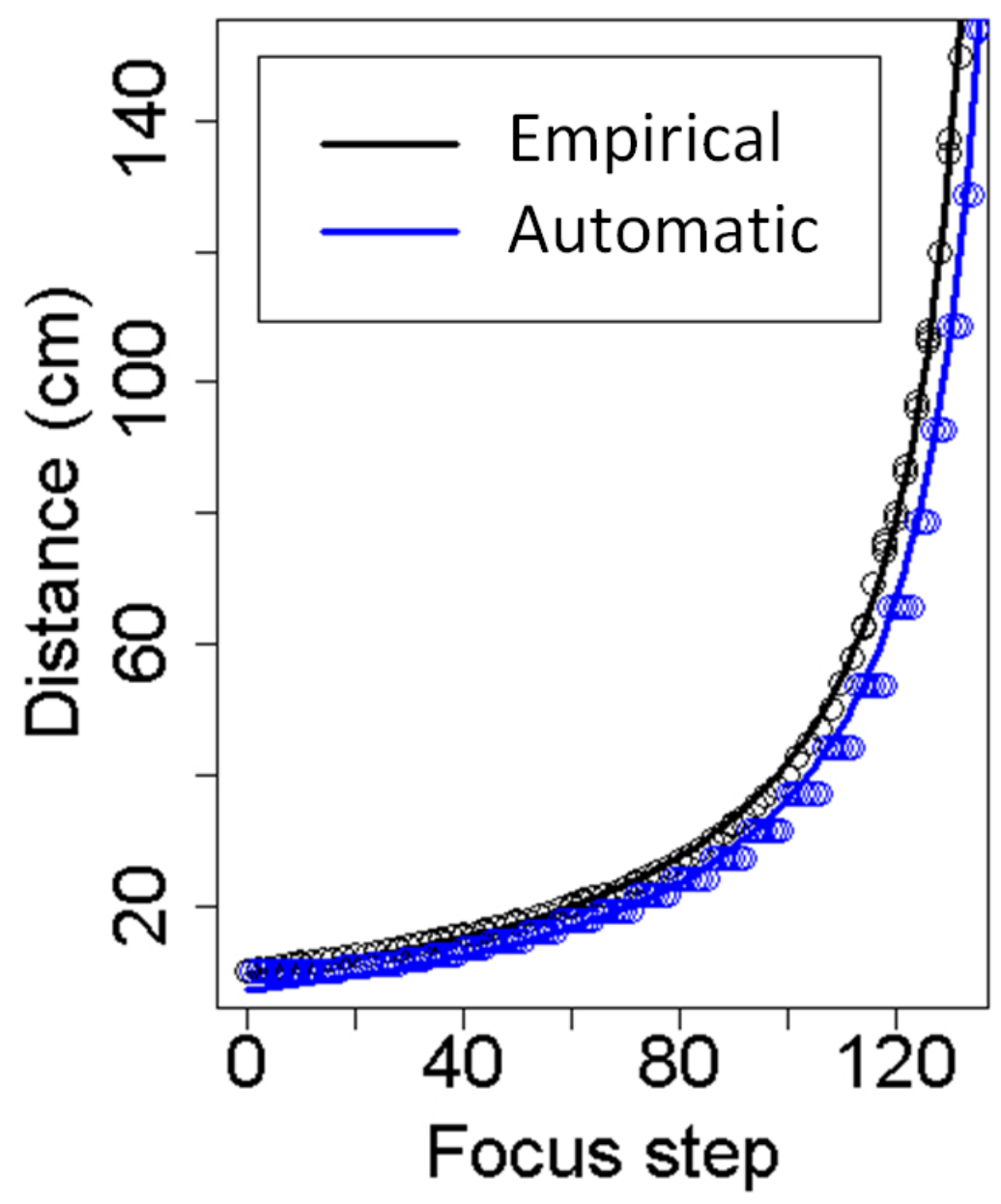

Figure 2.2. The distance estimation accuracy of an empirical and an automatic depth profile. For visual purposes the estimates were aligned at zero, by subtracting the background distance estimate. 


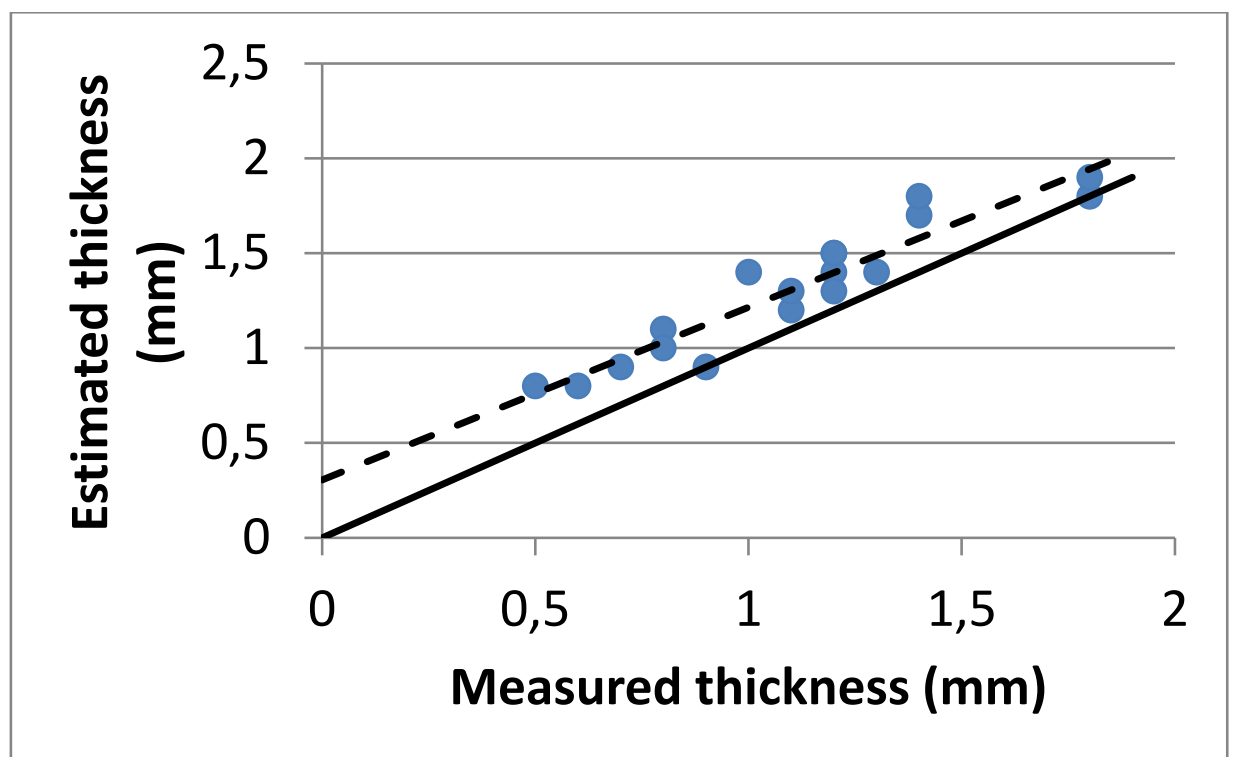

Figure 2.3. Measured (with caliper) versus estimated seed thickness, the solid line is the $1: 1$ ratio line, the dashed line is the regression line $\left(n=18, r^{2} a d j=0.89\right)$.

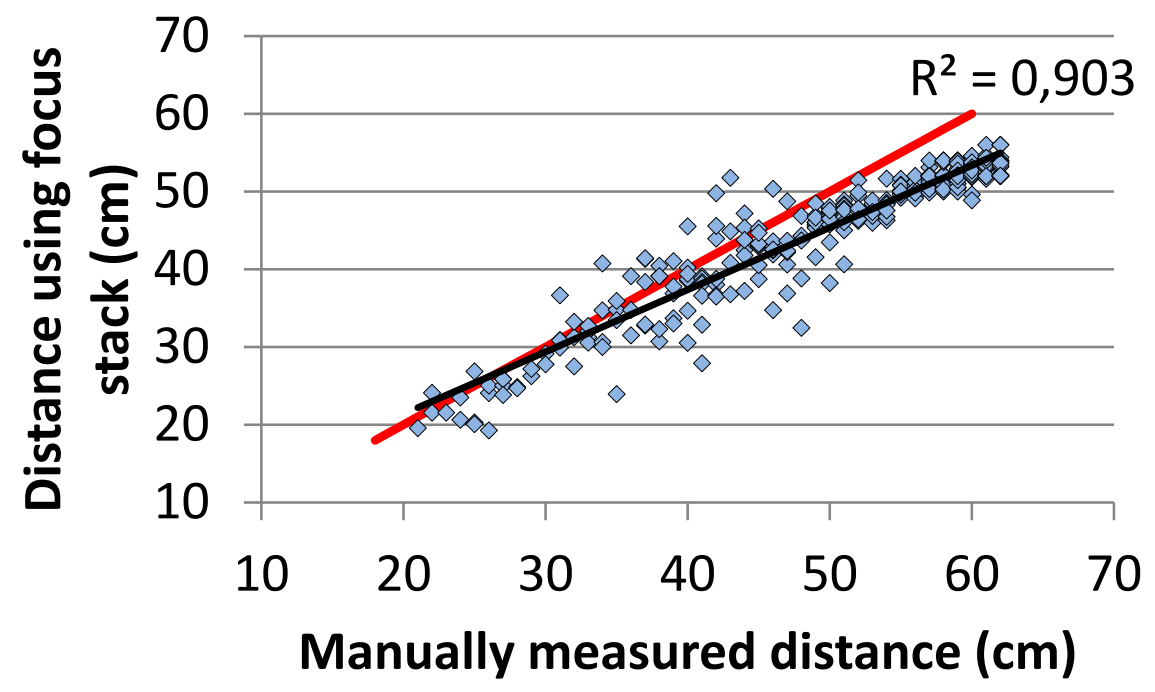

Figure 2.4. The comparison of manually measured vegetation height and depth from focus measured vegetation height. The red line is the 1:1 line, the black line is the trend line. 


\subsubsection{Application to saltmarsh vegetation structure in the field}

We compared three prominent salt marsh species in the field, and found clear differences in depth from focus. Low densities of Spartina cause sharp contrast in height with the substrate. Atriplex has a more dense structure, resulting in less height variation. Elytrigia has a very smooth structure with only very small variations in height (figure 2.5). This is also reflected by the statistical quantification of structure. All structure indicators show large differences between Spartina and Elytrigia with Atriplex in the middle (figure 2.6). The difference between Spartina and both others are statistically significant for the spatial autocorrelation $(n=26$, $F=3.605, p=0.043)$ and the tortuosity $(n=26, F=6.179, p=0.007)$. The RMSZ shows a similar pattern but is not significant $(n=26, F=2.934, p=0.073)$. The Moran-I of Spartina and Atriplex are more similar, and no significant differences were observed $(n=26, F=3.234, P=0.058)$. 


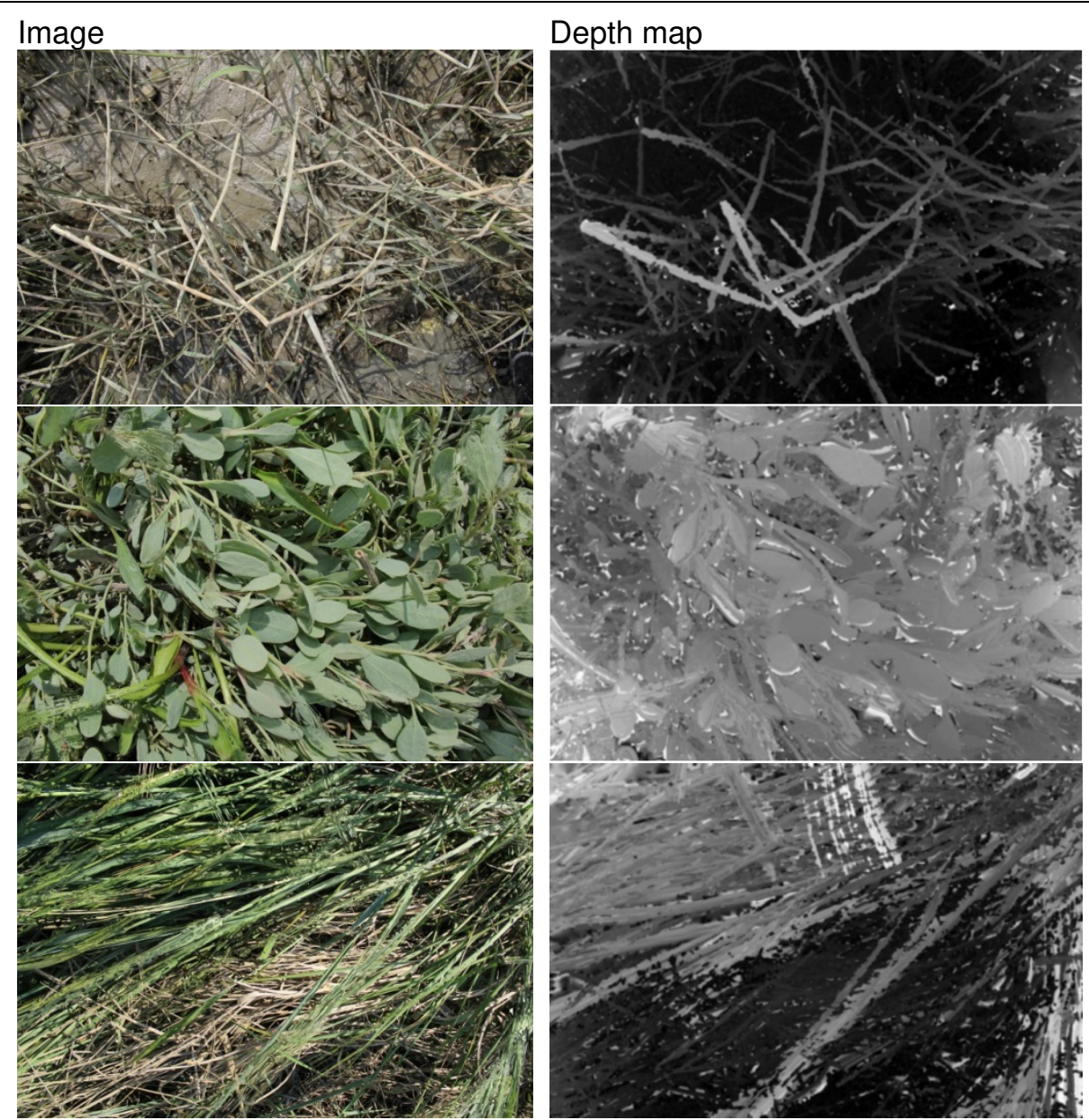

Figure 2.5. Depth maps of different species. Pixels further away from the camera are darker in the depth maps (right). The top two images show Spartina, the middle images show Atriplex, and the bottom images show Elytrigia. 

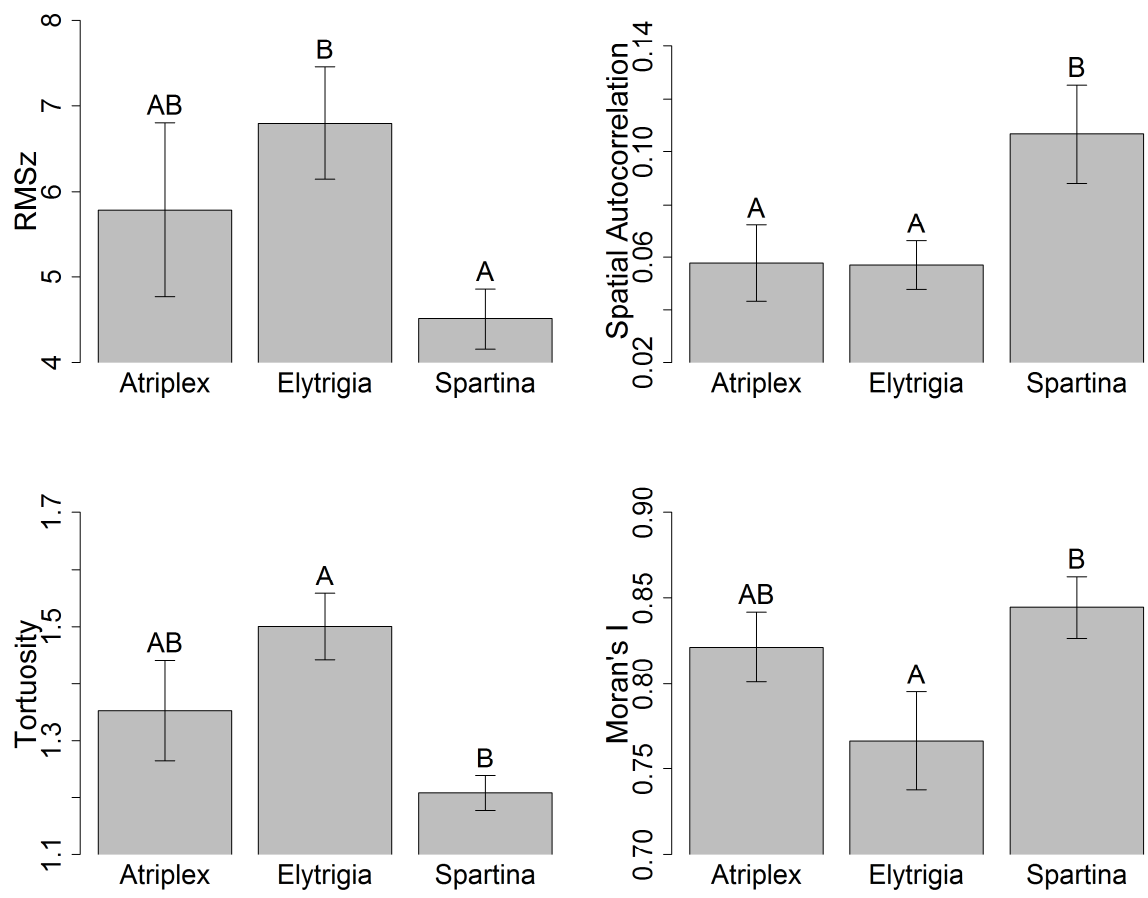

Figure 2.6. Differences in structure between three plant genera, expressed by multiple structure measures. Error bars are $\pm 1 \mathrm{SE}$. RMSz stands for root mean square error of the elevation $(z)$. 


\subsection{Discussion}

We demonstrated that depth from focus can be used to create $3 d$ representations of high accuracy and resolution. We showed how it can be used to characterize vegetation structure in salt marshes, and it looks promising for many other areas of study where habitat structure is important. Macro fauna studies could greatly benefit, for example the dependence of caterpillars on microhabitat structure can now be quantified.

\subsubsection{Validation}

The basis of this technique is fitting a thin lens function through calibration data. Although an analytical solution is possible it requires detailed technical knowledge of the lens. By fitting a thin lens function we can avoid requiring detailed information on every lens used, increasing the applicability and ease of use of depth from focus.

To test whether depth from focus can be used by ecologists, we performed extensive validation. We first compared both empirically and automatically drawn up distance profiles and found that depth from focus is able to accurately estimate the distance to an object both based on manual and automatic calibration, although the manual calibration outperformed the automatic procedure. We found that a curve fitted through a sufficiently large image stack, taken under controlled circumstances performed well. However, it is important to note that not all lenses support the automatic calibration feature, as not all lenses report the same information to the camera.

We performed further validation and manual measurements on small scale biological objects (grass seeds) with depth from focus and found a good agreement between measurements. Although there was a small but consistent overestimation by depth from focus, indicating it will remain important to properly calibrate it to a specific set of circumstances.

We validated the depth from focus techniques in vegetation structures, by comparing the estimated height with the actual height. The height estimation using depth from focus and the measured height showed a good agreement $\left(r^{2} a d j=0.903\right)$. At larger distances from the camera depth from focus seems to underestimate the distance. This is likely due to small errors in the depth profile, the translation list between focus step and distance. The overall good agreement shows that this technique can be used to create high quality $3 d$ representation of vegetation.

Our practical application in the field showed that there were significant differences between three salt marsh species, this underlines the ability of the technique to derive meaningful data on vegetation structure. We compared a standing grass, a 
lying grass and a small shrub species and found the largest structure difference between the grasses, the shrub like structure is in between. This indicates that this technique can be used to quantify the vegetation structure of salt marshes, it could therefore be valuable in wave mitigation studies where these data are required (Möller et al. 2014, Rupprecht et al. 2015). Depth from focus can provide a high resolution depth map, but another data processing step is required to quantify vegetation structure. We successfully applied spatial statistics to quantify vegetation structure but the performance of these statistics highly depends on the structure indicator. We compared several often used spatial statistics (following Bretar et al. 2013), however which indicator performs best is situation specific and the choice of indicator depends on the research goals.

\subsubsection{Applications and limitations}

Depth from focus is already used in laboratories, under highly controlled circumstances, to create detailed 3d images on a cell level (Gorthi and Schonbrun 2012). We applied it on a millimeter scale, using it to measure thickness of grass seeds, and we mapped vegetation structure on a centimeter and decimeter scale. The $\mathrm{cm}$ scale could be regarded as the operational limit of this method, although this strongly depends on the type of lens that is used. Technically the method is only limited by the focus precision of a camera, however as most cameras have a higher focus precision when focusing close to the camera, this method will be most precise when applied to an object close to the camera. The application of depth from focus to objects far away, i.e. moving towards the meter scale, will likely result in noisy data and have relatively poor resolution, limiting practical application. When a large scale analysis is desired, other techniques such as structure from motion or a terrestrial laser scanning might be more appropriate (Maas et al. 2008, Westoby et al. 2012). When a specific vegetation structure property, such as vertical vegetation structure for wave mitigation is under study, the relevant structure parameters should, if possible, be measured directly. As well performing methods such as the portable photo frame method are available (Möller 2006, Rupprecht et al. 2015). However for many small scale structure measures specialized measurement techniques are not available, in which case depth from focus can greatly improve measurement possibilities and resolution. Depth from focus exploits small scale color differences, by definition it cannot perform well on completely uniform surfaces. As a rule of thumb, if a normal DSLR camera cannot autofocus on a surface because it is to uniformly colored, depth from focus will likely perform poorly.

All methods that do not collect data instantly can suffer from wind influence; during our field measurements we found that for depth from focus it was sufficient to place 
a plexiglass (perspex) plate next to the camera, as a windbreaker, which was sufficient in the relatively small vegetation we measured. We encountered no problems with other environmental conditions such as sunlight or changes in light intensity, which are known to make 3d data acquisition difficult (Kazmi et al. 2014, $\mathrm{Li}$ et al. 2014). As with all passive collection methods, a minimum light level is required. If an area is severely shaded, and becomes almost completely black, it is no longer possible to determine its distance to the camera. We did not encounter this problem, but it might occur in more complex vegetation types. If this occurs it can be solved with an alternate light source. Depth from focus produces an envelope representation of a structure, not a full $3 d$ representation. This limitation cannot be overcome without combining multiple points of view. Only Stereo Vision and Structure From Motion offer partial or full 3d, because they inherently require multiple points of view. For some techniques such as Terrestrial Laser Scanning combining multiple points of view is common, however this approach can be applied to any technique to go from a $3 \mathrm{~d}$ envelope to full $3 \mathrm{~d}$.

\subsubsection{Possible improvements and developments}

A number of developments may improve the performance of the depth from focus method. A more advanced correction for changes in pixel size at larger focus distances would open possibilities for an even more precise quantification of micro habitat. In addition, a better hemispheric correction might improve results and decrease effects caused by observing from a single viewpoint. Another exciting possible future development is the usage of video rather than images. This would greatly improve the collection speed, and hence decrease the influence of wind and other environmental disturbances. As moving the lens causes vibrations, images might not be perfectly aligned anymore, but this may be overcome by using algorithms as used in stereo vision. In addition, special attention would be required to ensure that a position in the video can be related to a specific distance. If these difficulties could be overcome, it would greatly improve the applicability range and collection time.

\subsection{Conclusion}

Extensive validation showed that depth from focus can be used to map habitat structure on various scales ranging from millimeters to decimeters, and although applying it to a specific set of circumstances will require additional calibration, this method looks promising for many areas of study. Not only because it provides a quick and cheap method of collecting data, but also because it offers high resolution data performing well under field conditions. 


\section{Author contributions}

Oteman and Nieuwhof conceived the ideas and designed the methodology; Oteman collected the data; Oteman and Nieuwhof analyzed the data; Oteman led the writing of the manuscript. All authors contributed to writing of the manuscript and gave final approval for publication.

\subsection{Appendix}

\section{Supplementary: Distance estimation}

The distance estimation allows us to map the total number of pixels at each distance, however a pixel focused on a faraway object represents a larger surface area than a nearby pixel. In this study we focus on autocorrelation techniques depending on relative measures. As a consequence we do not require very accurate surface area estimates. However to correct for this effect the following formula can be used.

$$
s=2 d \cdot \tan \left(\frac{a \cdot \pi}{180}\right)
$$

Where $s$ is the surface area, $d$ is the distance and $a$ is half the camera viewing angle. This gives the total surface area of the image. The average surface area per pixel is calculated by dividing the calculated total area by the total pixel count. When multiplied with the number of sharp pixels in an image, this gives the total surface area per distance at a certain focus step. 


\section{Chapter 3: Using remote sensing to identify drivers behind spatial patterns in the bio-physical properties of a saltmarsh pioneer}

B. Oteman ${ }^{1 *}$, E.P. Morris ${ }^{2}$, G. Peralta ${ }^{2}$, T.J. Bouma ${ }^{1}$, D. van der Wal ${ }^{1,3}$

${ }^{1} \mathrm{NIOZ}$ Royal Netherlands Institute for Sea Research, Department of Estuarine and Delta Systems, and Utrecht University, P.O. Box 140, 4400 AC Yerseke, the Netherlands.

${ }^{2}$ Department of Biology, Faculty of Marine and Environmental Sciences, University of Cádiz, 11510 Puerto Real (Cádiz), Spain

${ }^{3}$ Faculty of Geo-Information Science and Earth Observation (ITC), University of Twente, P.O. Box 217, 7500 AE Enschede, The Netherlands.

\section{Published in:}

Remote Sensing, 2019, 11(5), 511, https://doi.org/10.3390/rs11050511

Abstract: Recently spatial organization in salt marshes was shown to contain vital information on system resilience. However, in salt marshes, it remains poorly understood what shaping processes regulate spatial patterns in soil or vegetation properties that can be detected in the surface reflectance signal. In this case study we compared the effect on surface reflectance of four major shaping processes: flooding duration, wave forcing, competition and creek formation. We applied the ProSail model to a pioneering salt marsh species (Spartina anglica) to identify through which vegetation and soil properties these processes affected reflectance, and used in situ reflectance data at the leaf and canopy scale and satellite data on the canopy scale to identify the spatial patterns in the biophysical characteristics of this salt marsh pioneer in spring. Our results suggest that the spatial patterns in the pioneer zone of the studied salt marsh are mainly caused by the effect of flood duration. Flood duration explained over three times as much of the variation in canopy properties as wave forcing, competition or creek influence. It particularly affects spatial patterns through canopy properties, especially leaf area index, while leaf characteristics appear to have a relatively minor effect on reflectance. 


\section{Graphical abstract:}

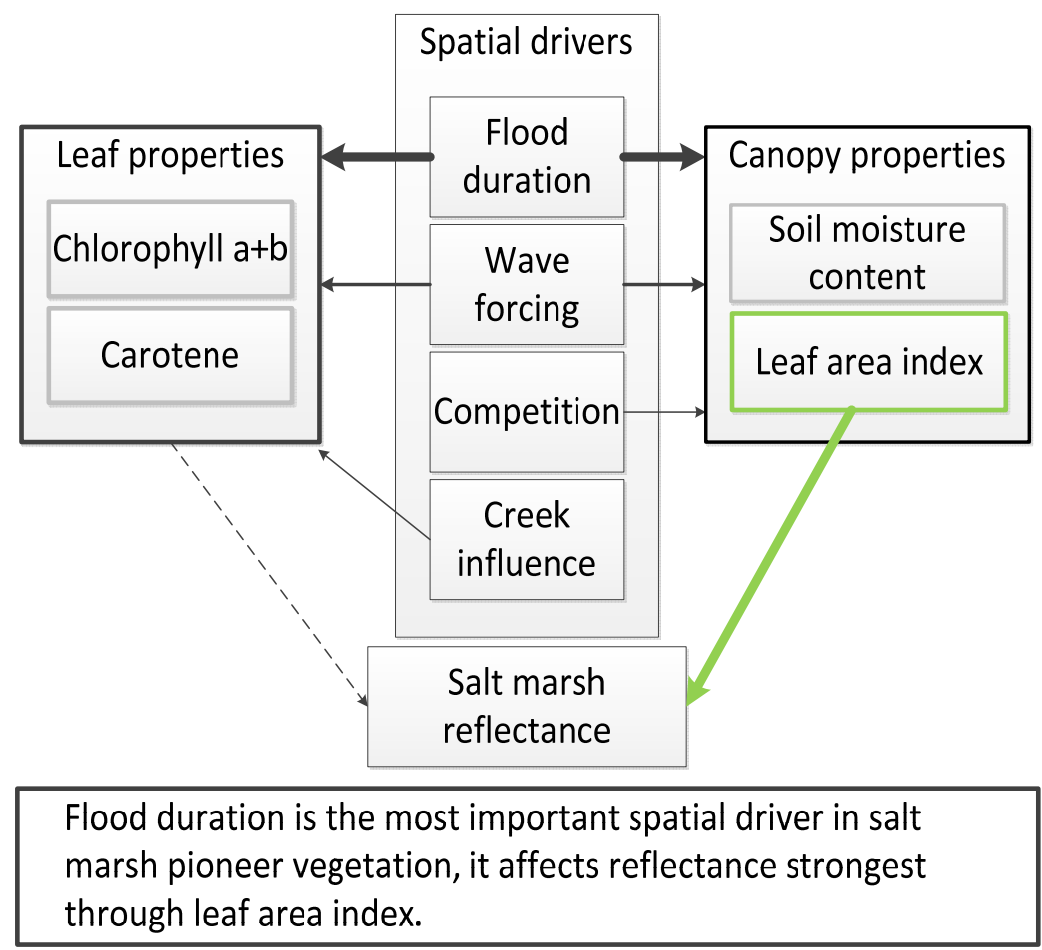

Keywords: ProSail; Salt marsh; Spartina; reflectance; Spatial patterns; Flood duration

\subsection{Introduction}

Analyzing spatial patterns has since long been recognized as an important method to understand the mechanisms organizing ecological systems (Legendre and Fortin 1989). Understanding the processes that generate ecological spatial patterns in plant communities is historically considered a major goal of community ecology (Bertness and Ellison 1987), which recently gained renewed attention when it was suggested that spatial patterns could increase the precision in predicting sudden critical transitions (Kéfi et al. 2013). An example of this can be found in salt marshes where spatial patterns were found to contain vital information 
on system resilience (van Belzen et al. 2017). Despite its usefulness, the underlying biotic and abiotic mechanisms causing these patterns remain poorly understood (Marani et al. 2013), which limits the interpretation of spatial patterns. In addition, it is often unclear how these underlying mechanisms are expressed and become visible. In this study we aim to improve the interpretation of spatial patterns by investigating which biotic and abiotic mechanisms have created the spatial patterns, and by examining through which vegetation characteristics these mechanisms become visible as spatial patterns. This will increase our general understanding of ecosystems and improve our ability to monitor their stability.

Spatial patterns can be defined as a pattern in the spatial distribution of a variable, in this study we focus on vegetation reflectance, and hence with spatial pattern we mean the spatial distribution of similarities and dissimilarities of vegetation reflectance. In general, two types of vegetation based spatial pattern studies can be distinguished: i) studies focusing on the effect of an (often abiotic) parameter on vegetation zonation (i.e., zonation of different plant species) and ii) studies focusing on how vegetation properties affect spatial patterning (i.e. patterns in a single species). In the first case, studies generally focus on the effect of a single parameter (e.g. nutrient composition, salinity or competition) on vegetation zonation. Here the spatial pattern changes as a result of changes in composition of the species. In the second case, studies can focus on the effect of changing vegetation properties on spatial patterns, for example the effect of drought, and corresponding low water content in plants (Samanta et al. 2010, Caccamo et al. 2011, Murad and Islam 2011) or changes in biomass in response to salinity (Lobell et al. 2010, Aldakheel 2011). This changes the appearance of plants, which changes the spatial pattern. Our study falls in the second category as we are interested in the vegetation properties that are affected by biotic and abiotic processes. In this study we focus on the spatial variation of biophysical properties of a single species, and do not look at vegetation zonation.

To understand the stability of services provided by ecosystems and the resilience of such a system, using spatial patterns, analyses have to be performed at a large spatial scale, which is made feasible with remote sensing (Belluco et al. 2006). Satellite data offer a sufficient spatial scale and synoptic coverage that cannot easily be obtained through field observations (Belluco et al. 2006). The effects of vegetation properties on optical reflectance on a scale that affects satellite data, and hence affects spatial patterns, are traditionally studied in two different ways: 
either through vegetation indices (VI's) linked through correlation with physical properties or through physical models (Berger et al. 2018). Vegetation indices are widely used, whereas physical models are most often used in agriculture. A VI is generally directly related to in situ biophysical properties or environmental properties, and is influenced by biophysical properties of the canopy and leaves. VIs are also sensitive to Isoil background (e.g., grain-size, moisture content, organic matter), chlorophyll content or spatial orientation of leaves (Tripathi et al. 2012), this broad sensitivity makes it difficult to establish how each characteristic is being affected, as the VI is a result of the combination of all of them. Physical models allow for a more in-depth analysis, and can be used to establish the effects of individual vegetation characteristics on reflectance. Physical models use properties of the object under study as parameters and apply the physical laws to simulate reflectance. Often these models are then inverted to estimate object properties from reflectance (Jacquemoud 1993, Bicheron and Leroy 1999, Tripathi et al. 2012) (for a more extensive introduction on reflectance modelling and advantages over correlation models see (Berger et al. 2018)). This modelling approach allows each modelled parameter to be studied separately and its individual effect size can be established, which is a major advantage over correlation studies based on simple Vl's (see: Berger et al. 2018). As our in-depth study of the effects of biotic and abiotic processes on vegetation properties depends on isolating the effects of vegetation properties to study their effect on reflectance, we will apply the physical model ProSail (see the method section for further details).

Salt marshes provide valuable ecosystem services; they support local fisheries by providing a refuge for juvenile fish (Boesch and Turner 1984, Deegan et al. 2002, Chmura 2011), economic services such as increased tourism (Henderson and Lewis 2008, Chmura 2011), reduced nutrient loading in coastal waters (Chmura 2011) and wave mitigation and flood protection (Möller 2006, Barbier et al. 2008, Möller et al. 2014). The ecosystem services of tidal salt marshes have even be said to parallel those of mangrove forests (Chmura 2011), which are known to be extremely valuable (Rönnbäck 1999, Jerath et al. 2016, Rizal et al. 2018). Hence, understanding the processes that drive the development of spatial patterns in marshes will help us to safeguard these ecosystems and the services they provide. The effect of vegetation properties on spatial patterns in this valuable ecosystem is still poorly understood. Previous studies mainly focused on the effects of vegetation zonation on spatial patterns (Bertness and Ellison 1987, Zedler et al. 1999, Sanderson et al. 2001, Silvestri and Marani 2004, Moffett et al. 2010, 
Pettengill et al. 2018). These vegetation zonation studies reported various influential factors, highly influential factors are: inundation time (Bertness and Ellison 1987, Pennings and Callaway 1992, Zedler et al. 1999, Silvestri et al. 2005), wave forcing (Neumeier and Ciavola 2004, Bouma et al. 2005, 2010, Fagherazzi et al. 2006, Callaghan et al. 2010), competition (Bertness and Ellison 1987, Bertness and Hacker 1994, Emery et al. 2001) and creek influence (Zedler et al. 1999, Sanderson et al. 2001, Silvestri et al. 2005, Xin et al. 2013, Zhao et al. 2016).

The aim of this paper is to gain insight into which factors drive spatial patterns in a mono-specific saltmarsh vegetation. Our primary aim is to describe the relative effect of four landscape shaping processes, i.e. flooding duration, wave forcing, competition and creek formation, on reflectance of a single salt marsh species. Our secondary aim is to identify through which vegetation properties these effects propagate to reflectance, using a radiative transfer model to simulate vegetation reflectance. As we aimed to model how spatial drivers affect reflectance, we focused on a single wide-spread species Spartina anglica, which is a common pioneer grass. $S$. anglica occurs nearest to the water, but is outcompeted at higher elevations (Bertness and Ellison 1987, Pennings and Callaway 1992, Bertness and Hacker 1994). This species faces multiple stressors and hence is likely to manifest differences in biophysical characteristics (e.g. leaf area index, chlorophyll content). In addition, it occurs in sufficiently large areas to be observable from a space borne platform. 


\subsection{Materials and Methods}

To investigate the effects of the drivers flooding duration, wave forcing, competition and creek formation on spatial patterns in salt marshes we looked at it at three levels. We (1) examined which vegetation properties are affected by these drivers, we (2) investigated how this translates to reflectance, and (3) used satellite data to map the large scale effects of the drivers to see how large scale spatial patterns are affected.

To examine which vegetation characteristics are affected by the mentioned drivers (1) we compared the influence of these drivers with in situ measurements using linear regressions. To translate between vegetation properties and reflectance (2) we used the radiative transfer model ProSail (Jacquemoud and Baret 1990). This model was calibrated with detailed in situ vegetation measurements, and validated with independent vegetation measurements. The linear regressions of step 1 were not used in this model. We used this model to evaluate the effect of plant and canopy characteristics on vegetation reflectance, by simulating spectra using the range of the in situ measured values and examining the size of their effect on the simulated spectra. Finally, (3) we applied the model to a satellite image and combined it with the estimated effects of the drivers behind spatial patterns to examine their impact at a large scale.

For the first analysis (1) we used the entire range where the study species $(S$. anglica) occurs, as this does not depend on optical data. For the other analyses (2 and 3), where optical data is used, we selected only plots where the study species is dominant, to avoid mixing reflectance of multiple species.

\subsubsection{Area}

Our main study area is a Dutch salt marsh named 'Paulina' in the Westerschelde estuary (Lat:51.35', Lon: $3.718^{\circ}$ ). The site is tide-dominated, and experiences a semi-diurnal tidal regime, with a spring tidal range of $\pm 4.5 \mathrm{~m}$. The site faces northeast, and is therefore relatively sheltered from the predominantly southwestern winds (Van der Wal et al. 2008b, Callaghan et al. 2010). The pioneer zone is dominated by common cord-grass (Spartina anglica), but other species as sea couch grass (Elytrigia atherica) and sea purslane (Atriplex portulacoides) also occur in the saltmarsh. Paulina saltmarsh is fronted by a ca $300 \mathrm{~m}$ wide mudflat area. The sediment in most of the estuary consists of sand and mud. The median 
grain diameter of the mudflat in front of Paulina is $0.097 \mathrm{~mm}$ (Van Der Wal and Herman 2007, Callaghan et al. 2010). The salinity of the water fluctuates throughout the season, for more details see: (Van Damme et al. 2005, Callaghan et al. 2010).

\subsubsection{In situ measurements}

Independent sets of in situ data were collected for calibration and validation in May 2015. To calibrate the model we collected data along three transects. Each transect started at the vegetation edge, adjacent to the water and was extended landward perpendicular to the water line, until Spartina no longer occurred. Additionally, we verified that the species no longer occurred over the next $10 \mathrm{~m}$ landward of the final point of each transect. Along the transects, a $1 \times 1$ meter plot was placed every 5 meters for sampling. The location of the center of the plot was recorded with a differential gps (dGPS). In each plot, vegetation cover, vegetation biomass, soil moisture content, reflectance and chlorophyll content were measured.

The vegetation cover was estimated for each plot in percentages using expert judgement. Alongside the $1 \times 1 \mathrm{~m}$ plot above ground vegetation biomass was sampled using a square area of $20 \times 20 \mathrm{~cm}$ (outside the $1 \times 1 \mathrm{~m}$ plot). The biomass samples were fresh weighed (FW), dried for a minimum of 4 days in an oven at 65 C and weighed again (dry weight, DW). Soil-moisture content was measured by taking samples of the top three $\mathrm{cm}$ of the soil using a syringe with the nozzle cut off. For each plot, this was sampled 3 times, samples were pooled and processed. These samples were fresh weighed (FW), freeze-dried for 72 hours and weighed again (DW), following (Wang et al. 2017).

Reflectance was measured using a TriOS Ramses 842D spectroradiometer. This device measures electromagnetic radiation between 320 and $950 \mathrm{~nm}$, sampling every $3.3 \mathrm{~nm}$ with $0.3 \mathrm{~nm}$ accuracy. Measurements are performed with a specially designed rig to hold the spectroradiometer stable at 2 meters above the sediment surface. The measurement radius on the ground then becomes a circle with a diameter of $20 \mathrm{~cm}$. This was done five times per plot, in a quincunx (dice five) pattern to avoid overlap and hence pseudo-replication. A reference measurement was taken prior to each measurement with a piece of white Styrofoam. Styrofoam is known to have a stable reflectance that represents incoming radiance (Kromkamp et al. 2006). We attempted to minimize the time between measurement and reference. If the light intensity changed noticeably between 
reference and measurement, both were taken again. All measurements were manually checked for errors, reference measurements were used to correct for irradiance following (Kromkamp et al. 2006).

Similarly to the reflectance measurements at canopy level, additional spectral measurements were taken at leaf level. To do so, 10-30 top leaves were randomly collected within each plot. This set of leaves was arranged to form a surface of about $8 \times 10 \mathrm{~cm}$. Special care was taken to ensure leaves were not upside down, and clean. This surface was placed at exactly $16 \mathrm{~cm}$ from the spectroradiometer. At this distance only the center circle with a $1 \mathrm{~cm}$ diameter was measured. Every 'leaf surface' was measured 5 times, moving the measuring area to avoid overlapping of the measurements and taking a reference measurement in between two measurements. Further processing was similar to the measurements at canopy level.

After the leaf surface measurements, the leaves of every leaf surface were immediately frozen and brought to the lab for chlorophyll analysis. The chlorophylls $\mathrm{a}, \mathrm{b}$ and a-carotene were extracted using high-performance liquid chromatography (HPLC). For the HPLC procedure we follow (Van der Wal et al. 2008a). To measure chlorophylls the leafs were first freeze dried, and treated with a $10 \mathrm{ml}$ 90/10 acetone/water solution to extract a sample. After centrifugation $50 \mu \mathrm{l}$ of this extract was separated for pigments on a C18-column with use of reversed phase chromatography. Separation was based on the interaction of pigments between column and the tertiary gradient used. After separation, the pigments were detected by a Photodiode Array (PDA) and a fluorescence detector.

Additionally, along the same transects, leaves were collected (on 1 June, 2017), individually weighed and photographed. Leaves were placed on white paper with a millimeter grid and a glass plate was placed on top to ensure they were completely flat. After being photographed leaves were weighted (FW) dried individually at $55^{\circ} \mathrm{C}$ for 5 days and weighted again (DW). Their surface area was calculated from the photographs, which allows for the calculation of weight and water content per leaf surface area. Leaf water content was not measured in the same period as the other measurements, it was therefore only used to fix the leaf water content parameter in the model to a reasonable number.

For validation, 10 additional plots were measured in the same study area in May 2015. Vegetation biomass and reflectance were recorded, similar to the measurements at the calibration plots. 


\subsubsection{Spatial drivers}

As spatial drivers, we investigated four factors expected to have a large effect on salt marshes patterns; flood duration, wave forcing, competition and creek influence.

Flood duration or inundation time is often estimated from elevation, and is expected to affect spatial patterns in salt marshes, likely because it causes stress in plants. Previous works has shown that flood duration is an important driver behind spatial patterns (Bertness and Ellison 1987, Pennings and Callaway 1992, Silvestri et al. 2005), and a small change in inundation time can cause large shifts in competitive interactions (Zedler et al. 1999), which in turn can affect reflectance of our study species. A map of flooding duration (resolution $20 \mathrm{~m}$ ) of the Westerschelde estuary was provided by Rijkswaterstaat (Paree 2017). This map is based on elevation data collected in 2016 using airborne laser altimetry, with an accuracy of $\pm 10 \mathrm{~cm}$, in combination with tide modelling, the tide model has a maximum error margin of $\pm 3 \%$ (see (Paree 2017)). We resampled this map to a 5 meter resolution using bilinear interpolation.

Wave forcing can shape salt marshes (Callaghan et al. 2010) and facilitate sediment resuspension (Fagherazzi et al. 2006). Vegetation attenuates waves (Neumeier and Ciavola 2004, Bouma et al. 2005, 2010), decreasing their influence further inland, which may create a spatial pattern. To quantify wave mitigation we used the distance to the bare mudflat (i.e. the seaward vegetation edge). This was calculated with the Euclidean distance tool in ArcGIS 10.1. The seaward vegetation edge was derived from the RapidEye image (see space borne data), a NDVI threshold of 0 was used to distinguish between marsh and unvegetated foreshore (i.e., the mudflat).

Competition is also known to be an important driver for plant zonation in salt marshes (Emery et al. 2001). Transplantation experiments indicated that facilitation and competition play an important role in determining spatial patterns in salt marshes. These experiments showed that species adapted to grow at low elevation (such as our study species) are competitively excluded from higher elevations, even though the high intertidal zone provides a more suitable habitat (Bertness and Ellison 1987, Bertness and Hacker 1994). The effect of inter-species competition was quantified using the distance to another major vegetation type. 
For this a vegetation map of 2010 by Rijkswaterstaat was used (Tolman and Pranger 2012), based on aerial photographs and field determination. The vegetation was recorded in vegetation types, but also included an estimated cover of our study species. An area was considered dominated by Spartina anglica when more than half of the total vegetated surface area was covered by this species. The distance to non-Spartina vegetation was calculated using the Euclidean distance tool in ArcGIS.

Creeks are also known to be a shaping feature (Sanderson et al. 2001, Silvestri et al. 2005, Xin et al. 2013), with a significant influence on soil properties and sediment accretion rates (Zhao et al. 2016), which in turn contributes to vegetation zonation (Zedler et al. 1999). The distance to the nearest creek is often used to quantify their influence (Silvestri et al. 2005, Xin et al. 2013, Zhao et al. 2016). Creeks were manually traced from a high resolution $(0.25 \times 0.25 \mathrm{~m})$ aerial photograph from 2016 provided by Rijkswaterstaat. Only creeks larger than $75 \mathrm{~cm}$ (3 pixels) were recorded. The distance to the nearest creek was calculated using the Euclidean distance tool in ArcGIS.

The four maps representing the effects of the four spatial drivers all had a resolution of $5 \mathrm{~m}$ and were compared with the in situ measured vegetation properties to identify which vegetation characteristics were most affected by the spatial drivers. For this analysis spatial driver information was extracted from the maps at the dGPS coordinates of the measured plots. Linear regression models, using the Im function in $r$, were used to compare the different spatial drivers with the in situ vegetation properties. 


\subsubsection{Model}

Physical models are often used to simulate reflectance and, after inversion, can be used to extract biophysical characteristics from reflectance (Jacquemoud 1993, Bicheron and Leroy 1999, Tripathi et al. 2012). A major advantage of using a physical model is the possibility to investigate which vegetation property affects spatial patterns. An often used physical model is 'ProSail', which is the combination of the leaf reflectance model 'Prospect' (Jacquemoud and Baret 1990) and the light scatter model in layers of leaves 'Sail' (Verhoef 1984). The combination of these two models is still improving, and it is often used due to its general robustness and because its inversion is known to perform well (Jacquemoud et al. 2009). We used the 'HSDAR' r-package to apply the ProSail model (Lehnert 2016). See table 3.1 for a complete overview of the model parameters. HSDAR uses the Fortran version of ProSail 5b, based on Prospect 5 and 4Sail. Using ProSail we simulated spectra between 400 and $2500 \mathrm{~nm}$.

The reflectance model 'Prospect' requires detailed information on leaf structure (structure parameter, N), water content parameters (equivalent water thickness, $\mathrm{Cw}$ ), dry matter content $(\mathrm{Cm})$ and chlorophyll contents (chlorophyll $\mathrm{a}+\mathrm{b}(\mathrm{Cab})$, carotene (Car) and brown pigment contents (Cbrown)). The leaves collected along the transect were used to calibrate Prospect, The Cab parameter was obtained from the chlorophyll- $a+b$ values. The Car parameter was estimated from the carotene values. For our model brown pigments were ignored. The average water content per leaf area was calculated from the photographed and dried leaf samples. The equivalent water thickness $(C w)$ is then calculated using the corresponding formula ( (FW-FD)/Area, see: (Bowyer and Danson 2004, Mobasheri and Fatemi 2013)). Average leaf equivalent water thickness (Cw) was found to be $0.0198 \pm 0.0043 \mathrm{~cm}(n=50)$. The leaf samples were also used to estimate the dry matter content per area $(\mathrm{Cm})$, this was found to be $0.0092 \pm 0.0025$ $\mathrm{g} / \mathrm{cm}^{2}(\mathrm{n}=50)$. The internal leaf structure parameter $(N)$ was fitted using only the 'Prospect' model, in combination with the leaf level spectra. Initially in situ spectra showed a higher baseline than the simulated spectra, hence a first degree polynomial conversion baseline was fitted using the spc.fit.poly function from the 'hyperSpec' r-package (Beleites 2017). The parameter $N$ was estimated as the value of the lowest RMSE, which was 1.5. This is precisely the value that the authors of the Prospect model predicted as best to describe monocotyledons (Jacquemoud and Baret 1990) such as Spartina. 
The 'Sail' model simulates vegetation canopy reflectance from the soil water content, the Leaf Area Index (LAl) and leaf angle distribution (lidf), solar, observation zenith and relative azimuth angle. Dry/wet fraction (psoil) is used to scale the brightness of the soil, using a linear mixture of standard spectra of dry and wet soil, respectively. Dry/wet fraction psoil=1 is used for dry soils, whereas psoil=0 is used for wets soils. In situ gravimetric dry/wet soil fraction (=1-soil moisture content) were obtained from freeze-drying the in situ soil moisture content samples. Leaf area index ( $\mathrm{LAl}$ ) was estimated from dry above ground biomass using the conversion provided by earlier research (Jensen et al. 1998). This study reported the linear relation between biomass and LAI for Spartina alterniflora as $y=634.95 x+5.4774$, where $y=L A I$ and $x=$ biomass. Spartina alterniflora is a close relative of our study species Spartina anglica. Spartina alterniflora is a cross bread between Spartina anglica and Spartina maritima that naturally occurred in 1870 (Meijden n.d.). These species are highly similar, we therefore assume the relation between biomass and LAI is similar too. Applying this conversion allowed for the estimation of leaf area index from above ground biomass, calibrated for Spartina (although a different species). Lidf describes the leaf angle distribution. Based on earlier research (Morris 1989) we assumed Spartina to be mostly a planophile, and set the lidf parameter correspondingly (lidfa $=1$, lidf $b=0$ ). The hotspot parameter was kept at 0 . 
Table 3.1, ProSail model parameters. The parameter abbreviation is the abbreviation used in HSDAR for the model parameter. The mean value is the average value of the in situ measurements, fixed means it is not derived from in situ measurements. The in situ range describes the range of values (minimummaximum) that occurred, and were used to simulate their effect on reflectance.

\begin{tabular}{|c|c|c|c|c|c|}
\hline Model & Parameter name & $\begin{array}{c}\text { Model } \\
\text { abbreviation }\end{array}$ & Mean value & In situ range & Source \\
\hline \multirow[t]{6}{*}{ Prospect } & Structure parameter & $\mathrm{N}$ & Fixed (1.5) & Fixed & $\begin{array}{c}\text { Fitted + } \\
\text { (Jacquemoud and Baret 1990) }\end{array}$ \\
\hline & Chlorophyll $a+b$ content & $\mathrm{Cab}\left(\mu \mathrm{g} / \mathrm{cm}^{2}\right)$ & 56.4 & $42.4-76.5$ & Chl samples \\
\hline & Carotenoid content & $\operatorname{Car}\left(\mu \mathrm{g} / \mathrm{cm}^{2}\right)$ & 3.421 & $2.398-4.579$ & Chl samples \\
\hline & Brown pigment content & Cbrown & N.A. & N.A. & - \\
\hline & $\begin{array}{l}\text { Equivalent water } \\
\text { thickness }\end{array}$ & $C w(\mathrm{~cm})$ & Fixed $(0.0198)$ & Fixed & leaf samples \\
\hline & Dry matter content & $\mathrm{Cm}\left(\mathrm{g} / \mathrm{cm}^{2}\right)$ & Fixed (0.0092) & Fixed & Leaf samples \\
\hline \multirow[t]{7}{*}{ Sail } & $\begin{array}{l}\text { Dry } \text { Wet soil fraction } \\
(=1 \text { - soil moisture } \\
\text { content })\end{array}$ & pSoil & 0.5340 & $0.4496-0.6214$ & Soil samples \\
\hline & Leaf area index & LAI & 0.706 & $0.003-1.215$ & $\begin{array}{c}\text { (Jensen et al. 1998) + } \\
\text { samples }\end{array}$ \\
\hline & $\begin{array}{l}\text { Type of leaf angle } \\
\text { distribution }\end{array}$ & Lidf & Fixed $(1,0)$ & Fixed & (Morris 1989) \\
\hline & Hotspot parameter & hspot & N.A. & N.A. & - \\
\hline & Solar zenith angle & Tts $\left(^{\circ}\right)$ & N.A. & N.A. & From timestamp \\
\hline & Observer zenith angle & Tto $\left(^{\circ}\right)$ & N.A. & N.A. & Always 0 \\
\hline & Relative azimuth angle & Psi $\left(^{\circ}\right)$ & N.A. & N.A. & From timestamp \\
\hline
\end{tabular}

\subsubsection{Model inversion}

The model was inverted using a look up table (LUT). We used a separate LUT for each vegetation characteristic, where all other vegetation properties were kept fixed on their average, and only a single variable varied, according to the range of values found in the field (See Table 3.1). We used very small increments and selected the closest matching value from each LUT. Spectra were compared using the spectral angle mapper (SAM) technique (Kruse et al. 1993), we used the HSDAR implementation of SAM.

To compare the LUT with the multispectral bands of the satellite image, the LUT was resampled, by multiplying each wavelength with the corresponding sensor band gains of the RapidEye satellite image (see 3.2.8). 


\subsubsection{Sensitivity modeled vegetation characteristics}

The sensitivity of the Prosail model in salt marshes was analyzed by comparing the effect of the minimum and maximum in situ values of a single parameter, while all other parameters remained constant. The sensitivity was checked by analyzing the effects of the four main model parameters: chlorophyll $a+b$ content, carotene content, soil moisture content and leaf area index (see table 1 for the tested value ranges). This method also provides insight into the relative contribution of leaf properties and canopy properties.

\subsubsection{Model validation}

To get insight into the model performance, we compared inverted in situ spectra, sampled at the calibration points, with in situ measurements. The model was inverted for a single parameter at a time. This provided insight into the effects of a single parameter on an in situ spectrum. To exclude mixed pixel effects, we used only plots dominated by Spartina (cover $>=95 \%$ : $n=22$ ). The spectra used for the validation process were not used for calibration. In addition we inverted spectra from a RapidEye satellite image (see 2.8) and compared the estimated values based on this inversion with in situ values collected at completely independent plots in the same study site.

The relative advantage of using a more advanced physical model when quantifying vegetation characteristics is tested by comparing the results of ProSail with a simple correlation based approach using NDVI. The NDVI was calculated using band 3 (red) and 5 (NIR) of the RapidEye satellite and compared with the modeled vegetation characteristics.

\subsubsection{Application to space borne data}

To test the applicability of the method to space borne data, we used a RapidEye satellite image of June $5^{\text {th }}, 2015$ with a spatial resolution of $5 \mathrm{~m}$. The image was atmospherically corrected using the Second Simulation of a Satellite Signal in the Solar Spectrum (6S) model, which is known to perform well (Kotchenova and Vermote 2007). This atmospheric correction model requires geometrical, 
atmospherical, sensor, spectral, ground reflectance and signal input (see table 3.2). The atmospheric profile was set to midlatitude summer.

In the RapidEye image, areas dominated by Spartina were selected, the ProSail model was applied to the reselected areas to estimate LAI. The spatial driver information was extracted at the center of every inverted RapidEye pixel. All analyses were performed on $5 \times 5$ meter pixels.

Again, linear regressions were used to compare the different spatial drivers with the estimated LAI (see method in section 2.4). The correlation between the effects of different drivers was tested with Pearson correlation tests. In addition a multiple linear regression was used to relate the estimated LAI with the spatial drivers in a single test to avoid explaining variation several times. This allowed us to establish the relative importance of each spatial driver. 
Table 3. 2, 6S atmospheric correction model parameters used for the RapidEye atmospheric correction.

\begin{tabular}{cc}
\hline Parameter & Setting \\
Month & 06, from satellite image \\
Day & 05, from satellite image \\
Solar zentih angle $(\mathrm{deg})$ & 28.91, from satellite image \\
Solar azimuth angle $(\mathrm{deg})$ & 171.91, rom satellite image \\
Sensor zenith angle $(\mathrm{deg})$ & 12.79, from satellite image \\
Sensor azimuth angle $(\mathrm{deg})$ & 281.32, from satellite image \\
Atmospheric profile & Mid latitude summer/winter, here summer \\
Aerosol profile & Maritime \\
Target altitude & Sea level \\
Sensor altitude & Satellite level \\
Spectral conditions & RapidEye gain, band 1-5 \\
Ground reflectance & Homogeneous surface \\
Directional effects & No directional effects \\
Input ground reflectance & Mean spectral value \\
& atmospheric correction with Lambertian \\
Atmospheric correction mode & a, Reflectance \\
Atmospheric correction target &
\end{tabular}




\subsection{Results}

\subsubsection{Effects of spatial drivers on in situ vegetation characteristics}

The direct effects of drivers behind spatial variation i.e. flood duration, wave forcing, competition and creek influence on in situ measured vegetation properties show that flood duration and wave forcing affected all vegetation characteristics, and their effects were strongest on chlorophyll $a+b$ and carotene content (figure 3.1). The level of significance is indicated by $p$, the $r^{2}$-adj is the adjusted $r^{2}$, as reported by the regression functions in $r$, and RMSE reports the root mean square error.

Competition is strongly correlated with soil moisture content and LAI. Nearness to creeks was only significantly correlated with chlorophyll $a+b$ and carotene content, but this correlation explained over $40 \%$ of the variation in both cases.

LAI seems to increase at a higher flood duration. However, a low flood duration only occurs at higher elevation, therefore this value is likely codependent on competition, which only occurs at higher elevation. The highest LAI values do not occur at high flood durations. The overall effect of flood duration on the leaf level is relatively small. 


\begin{tabular}{|c|c|c|c|c|}
\hline & $\begin{array}{c}\text { Flood } \\
\text { duration } \\
(\%)\end{array}$ & $\begin{array}{c}\text { Wave } \\
\text { forcing } \\
\text { (sea } \\
\text { distance m) }\end{array}$ & $\begin{array}{c}\text { Creek } \\
\text { influence } \\
\text { (distance } \\
\text { m) }\end{array}$ & $\begin{array}{c}\text { Competition } \\
\text { (distance } \\
\text { m) }\end{array}$ \\
\hline \multirow{2}{*}{$\begin{array}{l}\text { Chl- } \\
\text { ab } \\
\text { (ug/c } \\
\text { m2) }\end{array}$} & p:<0.001, r2-adj:0.41, RMSE: 6.56 & p:<0.001, r2-ad]:0.28, RMSE: 7.26 & p: $<0.001$, r2-adj:0.41, RMSE: 6.54 & p:0.08442, r2-adj:0.05, RMSE: 8.31 \\
\hline & 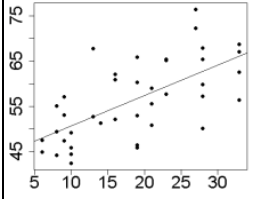 & & 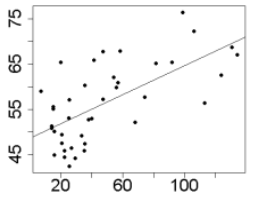 & 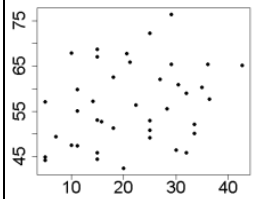 \\
\hline \multirow{2}{*}{$\begin{array}{l}\text { Caro } \\
\text { tene } \\
\text { (ug/c } \\
\text { m2) }\end{array}$} & $\mathrm{p}:<0.001$, r2-adj:0.42, RMSE: 0.47 & $\mathrm{p}:<0.001$, r2-adj:0.23, RMSE: 0.54 & p:<0.001, 12-adj:0.51, RMSE: 0.48 & p:0.10403, r-adj:0.04, RMSE: 0.6 \\
\hline & 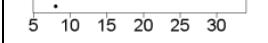 & 010 & 100 & 40 \\
\hline \multirow[t]{2}{*}{ LAI } & p:0.02007, 12-adj:0.11, RMSE: 0.31 & p:<0.001, r2-adj:0.24, RMSE: 0.29 & \multicolumn{2}{|c|}{ p:0.99755, 12-adj:-0.03, RMSE: 0.33 p:0.00532, 12-adj:0.17, RMSE: 0.3} \\
\hline & 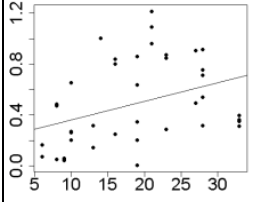 & 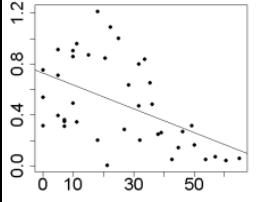 & $\begin{array}{ll}\therefore 0 & \\
20 & 60\end{array}$ & 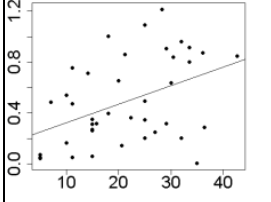 \\
\hline \multirow{3}{*}{$\begin{array}{l}\text { Dry/ } \\
\text { wet } \\
\text { Soil } \\
\text { frac } \\
\text { tion }\end{array}$} & p:0.01976, r2-adj:0.11, RMSE: 0.04 & p:0.00114, 12-adj:0.23, RMSE: 0.03 & p:0.87912, 12-adj:-0.03, RMSE: 0.04 & $\begin{array}{ll}4 & \text { p: }<0.001, \text { r2-adj:0.25, RMSE: } 0.03\end{array}$ \\
\hline & 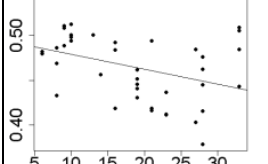 & 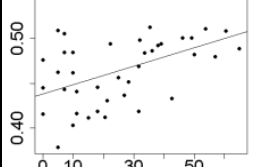 & 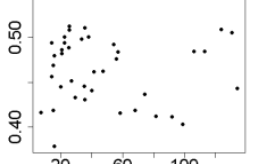 & $\overbrace{\vdots}$ \\
\hline & $\begin{array}{llllll}5 & 10 & 15 & 20 & 25 & 30\end{array}$ & 010 & $\begin{array}{c:c}20 & 60\end{array}$ & $10 \quad 20$ \\
\hline
\end{tabular}

Figure 3.1, the effect of spatial drivers on in situ vegetation properties. The leaf properties (chl-ab and carotene) are strongly affected by flood duration and creek formation. The canopy properties (LAI and the dry/wet soil fraction (1-soil moisture content)), are affected by flood duration, wave forcing and competition. Regression lines are shown for significant relationships: $p<0.05$ ). 


\subsubsection{Effects of vegetation characteristics on reflectance,}

\section{modelled sensitivity}

The spectral effects of four of the major model parameters, i.e., chlorophyll $a+b$, carotene, leaf area index and soil moisture content, were quantified via a sensitivity analysis on in situ data (figure 3.2). The chlorophyll $a+b$ content affects a limited range of wavelengths (i.e., 500 to $750 \mathrm{~nm}$ ), whereas the effects of carotene on the reflectance spectra were negligible. Hence, the latter was fixed to its average value. LAI and soil moisture content have a large influence on the entire range of the spectrum. LAl shows a decrease of reflectance at higher LAI values, which was expected as higher leaf coverage can absorb more light and hence lower reflectance. As expected, LAl changes the shape of the spectrum, whereas soil moisture content only increases or decreases the entire modelled spectrum. The contribution of leaf level reflectance (as modeled by 'Prospect') appears subordinate to the contribution of canopy level reflectance (as modeled by 'Sail'). 


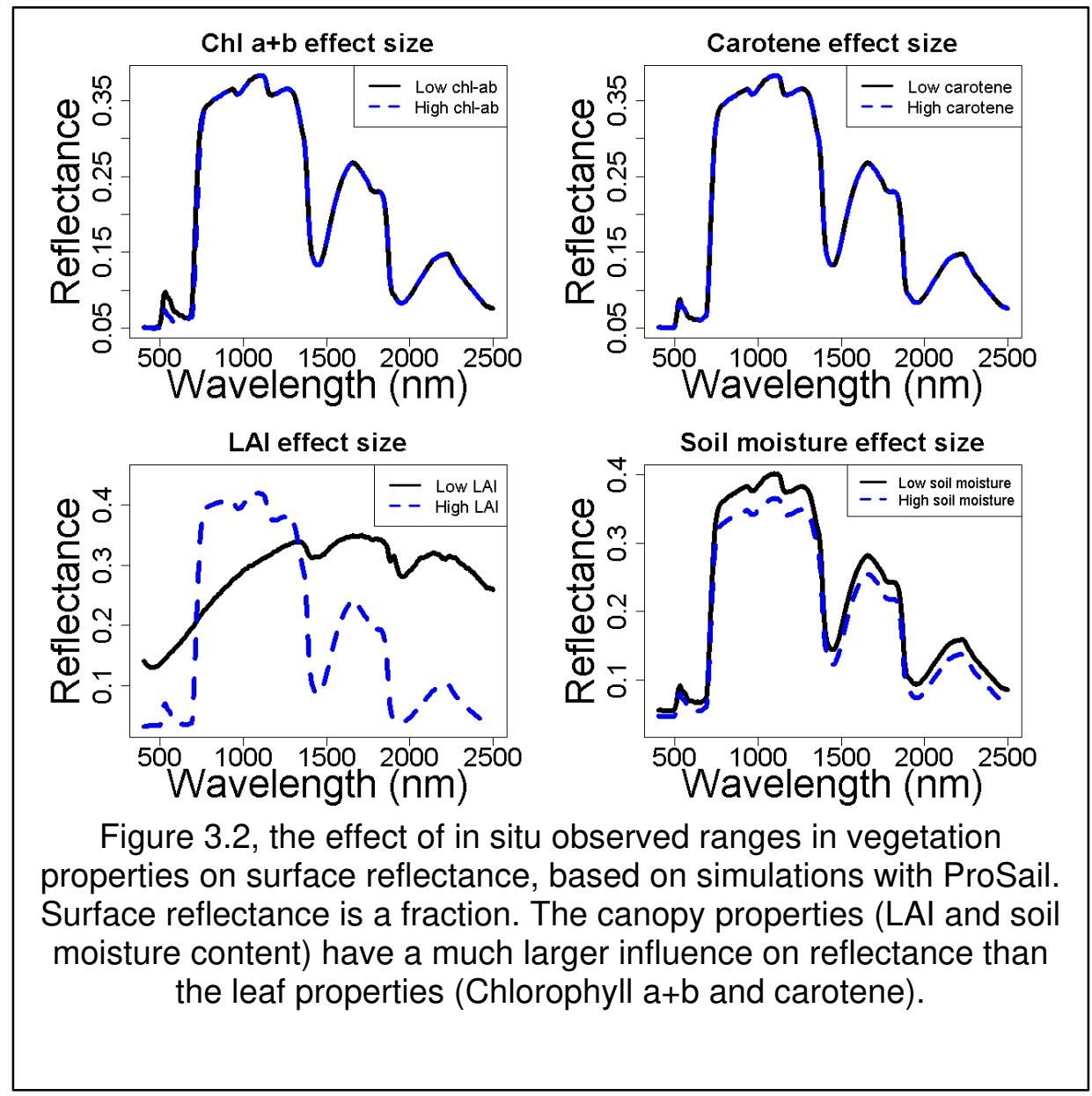




\subsubsection{Model validation}

To validate the model, we compared in situ measurements with estimated values based on the model inversion. The ranges used to construct the LUT's are shown in table 3.3. The model inversion shows that only LAI produces a significant, yet noisy, relationship between in situ measurement and model inversion, in both the calibration and the independent plots (figure 3.3). Chlorophyll was poorly estimated, and the soil moisture content was always estimates as completely dry. These were not taken into account any further, but rather fixed at their average values (see table 3.1).

The comparison between ProSail and NDVI shows that the estimated LAI is closely related to the NDVI (see figure 3.3) $\left(n=10, p<0.001, F=313, r^{2} a d j=0.97\right)$. However the ProSail model $\left(n=10, p=0.02, F=7.72, R^{2} a d j=0.4275\right)$ performs slightly better than NDVI $\left(n=10, p=0.036, F=6.318, R^{2} a d j=0.3714\right)$. Therefore, and for an improved comparability, we used the inverted model to estimate the leaf area index.

Table 3.3, model inversion look up table properties

\begin{tabular}{cccc}
\hline Parameter & Minimum & Maximum & Stepsize \\
\hline LAl & 0.001 & 3 & 0.01 \\
Chl-ab & & & \\
$\left(\mu \mathrm{g} / \mathrm{cm}^{2}\right)$ & 1 & 100 & 0.1 \\
pSoil & 0.1 & 1 & 0.001 \\
\hline
\end{tabular}

LAI: leaf area index; Chl-ab: Chlorophyll $a+b$ content; $p S o i l:$ dry/wet soil fraction (=1-soil moisture content). 
Chapter 3: Using remote sensing to identify drivers behind spatial patterns in the bio-physical properties of a saltmarsh pioneer
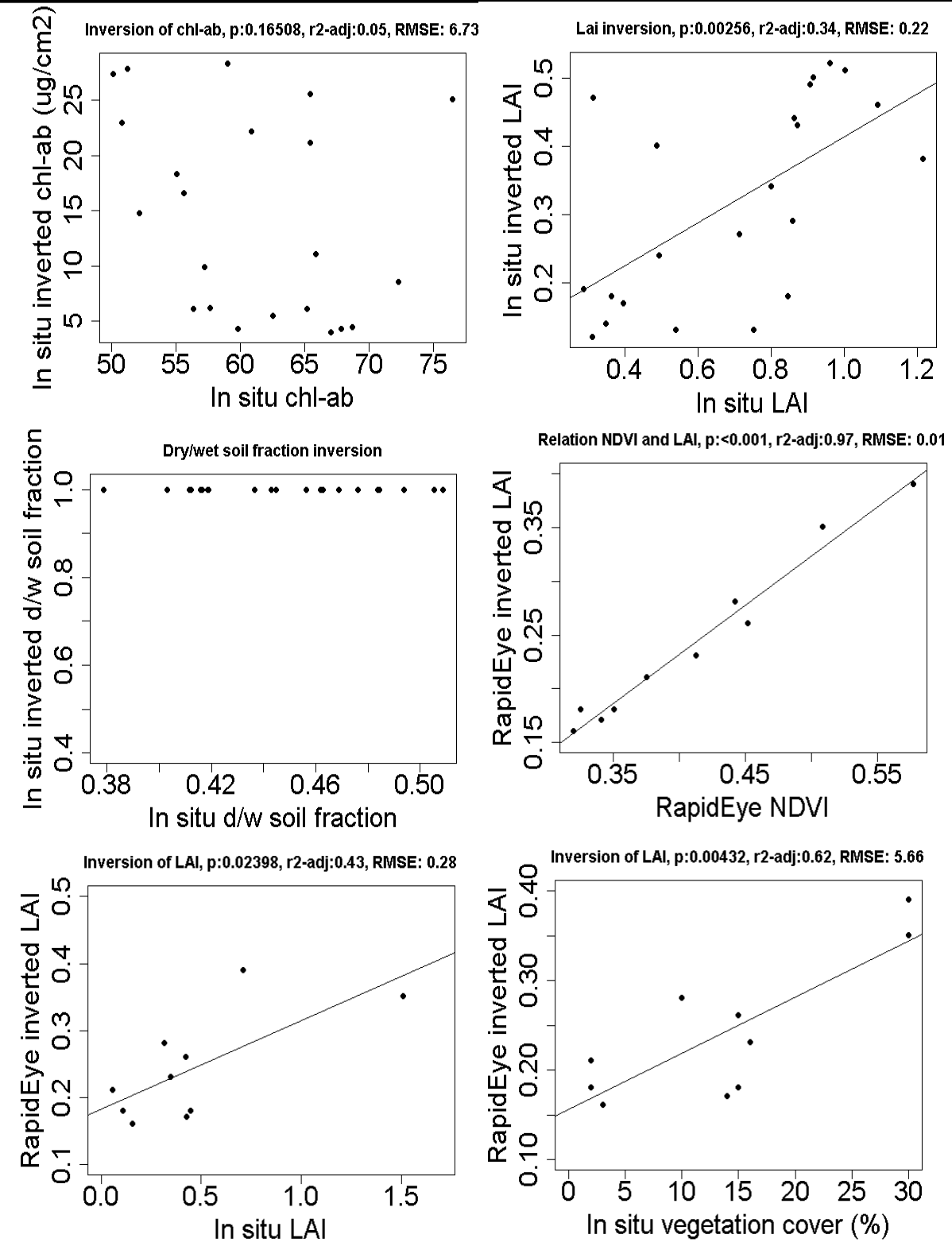

Figure 3.3, the first three graphs show the relation between in situ measurements and the model inversion of in situ spectra. The fourth graph shows the relation between the LAI inversion and NDVI both based on satellite spectra. The final two graphs show the model inversion of satellite spectra with in situ measurements of LAl and vegetation cover. Regression lines are shown for significant relationships $\mathrm{p}<0.05$ ). 


\subsubsection{Large scale effect of spatial drivers}

The inverted model was used to estimate LAI from satellite images, these data clearly show that flood duration has the largest influence on vegetation reflectance, followed by both wave forcing and distance to the nearest creek. Flood duration explains over three times as much variation as any of the other explanatory variables (figure 3.4). The data clearly show that a higher flood duration decreases LAI (figure 3.5). Although lower LAI values occur throughout the salt marsh, high LAl values only occur close to competitors and close to creeks. The wave forcing seems highest at a lower $\mathrm{LAl}$, the lowest wave forcing seems to occur along creeks high in the marsh, where LAl is high, although the relationship is noisy and explains only a limited amount of variation.

The Pearson correlation shows that all spatial drivers are significantly correlated (table 3.4). The multiple linear regression analysis supports that flood duration had the largest effect by far (table 3.5).

Flood duration explains $70 \%$ of the total variation, and shows clearly that higher flood duration decreases leaf area index. This seems contradictory to the effect at leaf level, where the pattern is unclear and explains only $11 \%$ of the total variation and the highest leaf area index values is found closer to the water. However when interpreting these results it is important to take into account that they are based on a different set of plots, as the leaf level analyses includes all plots, and the large scale analysis only includes plots where Spartina was dominant to avoid mixed pixel effects. 

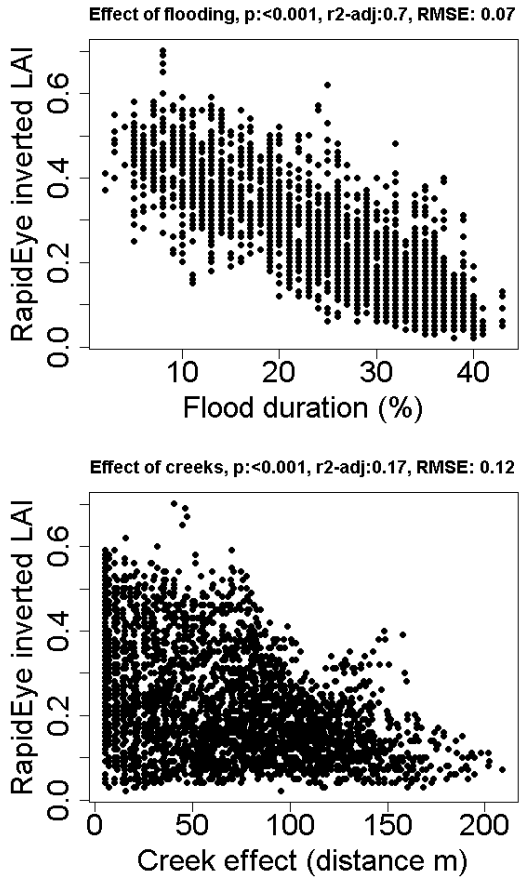
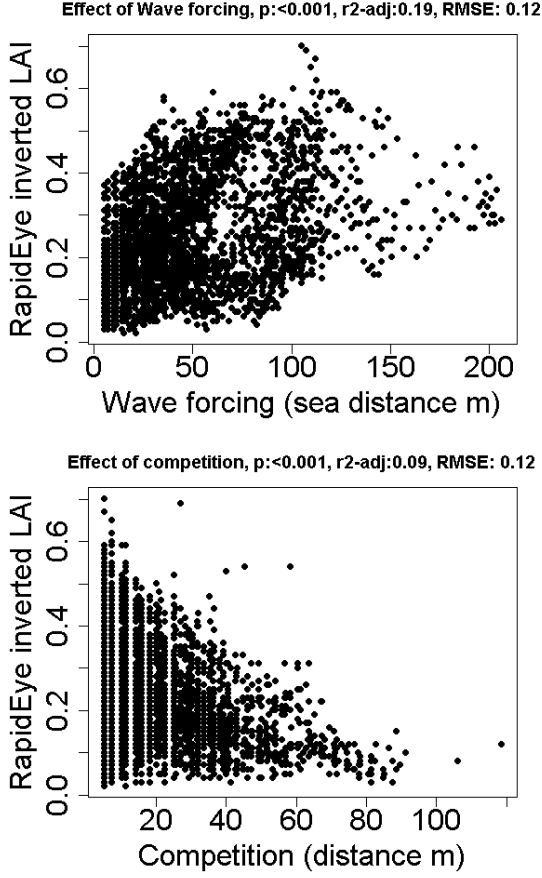

Figure 3.4, the large scale effects of the spatial drivers on salt marshes: relationships between drivers and Leaf Area Index (LAI) obtained from inversion of RapidEye satellite images. 


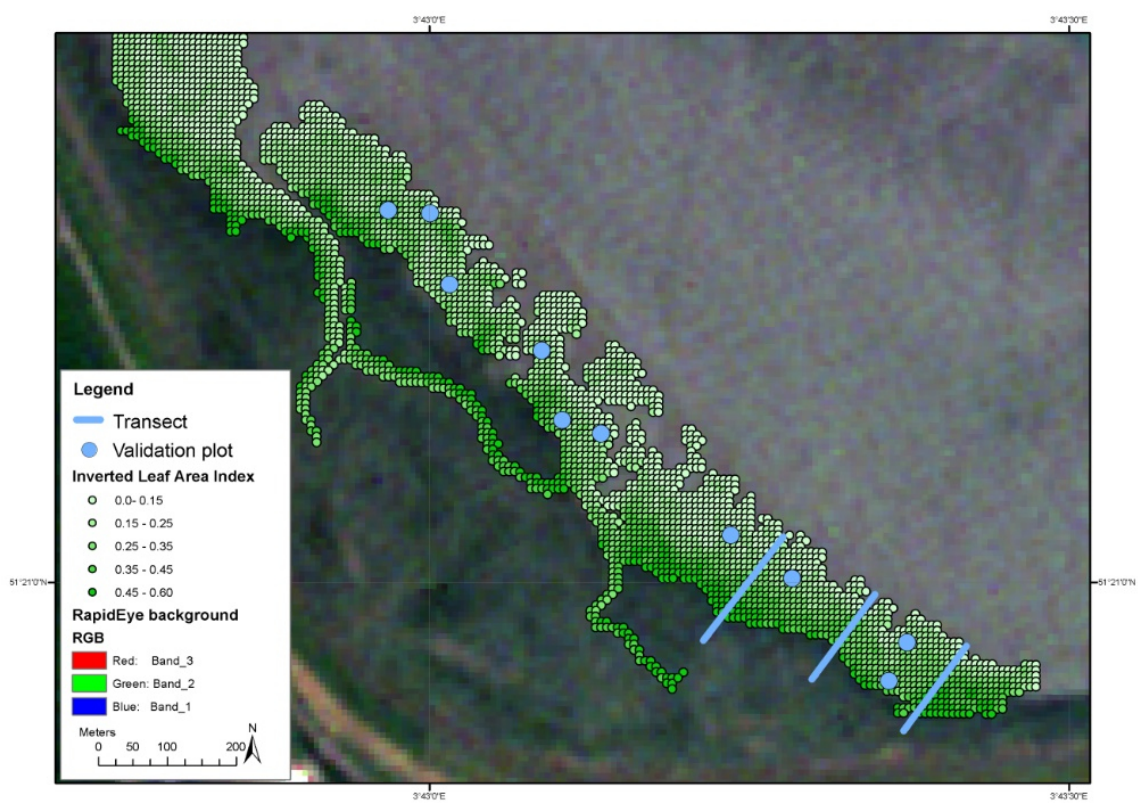

Figure 3.5, Spatial distribution of Leaf Area Index (LAI) estimated from the inversion of the ProSail model applied to a Rapid Eye image of June $5^{\text {th }}$, 2015.

Table 3.4, the spatial driver correlation coefficients, all spatial drivers were significantly correlated with $p$ values $<0.001$.

\begin{tabular}{ccccc}
\hline & Flood duration & $\begin{array}{c}\text { Wave } \\
\text { forcing }\end{array}$ & $\begin{array}{c}\text { Creek } \\
\text { influence }\end{array}$ & Competition \\
\hline Flood duration & 1.00 & -0.44 & 0.55 & 0.41 \\
Wave forcing & -0.44 & 1.00 & -0.30 & -0.08 \\
$\quad$ Creek & & & & \\
influence & 0.55 & -0.30 & 1.00 & 0.41 \\
Competition & 0.41 & -0.08 & 0.41 & 1.00 \\
\hline
\end{tabular}


Table 3.5, the contribution to the t value in the multiple-regression model relating spatial drivers with leaf area index.

\begin{tabular}{ccc}
\hline Spatial driver & Absolute contribution to t-value & Coefficient \\
\hline Flood duration & 67.743 & -0.01078 \\
Wave forcing & 9.124 & 0.00033 \\
Creek influence & 2.321 & 0.00007 \\
Competition & 0.900 & 0.00007 \\
\hline
\end{tabular}

\subsection{Discussion}

The aim of this paper was to gain insight into which factors drive spatial patterns in the mono-specific pioneer zone of a salt marsh, and through which vegetation properties these drivers affect reflectance. We found that in spring, in our study area flood duration has by far the largest effect on large scale spatial patterns and is the main mechanism behind these patterns. Flood duration is known to have a large effect on vegetation zonation, (Bertness and Ellison 1987, Pennings and Callaway 1992, Zedler et al. 1999, Silvestri et al. 2005). Here we showed that, in our study area, it is the driving factor behind spatial patterns in the mono-specific pioneer zone in spring. A higher flood duration was found to decrease the leaf area index. Flood duration explained $70 \%$ of the total spatial variation in LAI, over three times as much as the effect of wave forcing, which was found to be the second most important driver. Wave forcing is also known to be a shaping feature (Callaghan et al. 2010), and our result indicate that it indeed affected LAl. However, wave forcing only explains $19 \%$ of the total variation and the relationship appears noisy. The other two drivers behind spatial patterns, competition and creek influence, both show the same pattern: high LAI values only occur at low distances to competitors or creeks, low values occur everywhere. These drivers explain respectively $9 \%$ and $17 \%$ of the total variation. At this spatial scale it is clear that flood duration is most correlated to the differences in reflectance, overshadowing the effects of other factors.

It is important to note that this study is a first step towards understanding how drivers behind spatial patterns affect reflectance of the vegetation of single species and create spatial patterns. We focused on the monospecific pioneer zone of a single salt marsh in spring. Therefore our results have to be applied to other 
species and sites with other conditions, before conclusions can be drawn on salt marshes in a broader sense.

\subsubsection{Applicability to other vegetation zones}

In this study we focused on the pioneer zone of a salt marsh. Hence, it is possible that in the middle and high marsh other drivers become relatively more important, especially where differences in flood duration become relatively small. In our study, we focused on a single species (Spartina), the pioneer zone was dominated by this species. At higher elevations other species become more important. Previous research showed that even small differences in flood duration can already cause large differences in vegetation zonation, i.e. yielding different vegetation species or communities (Zedler et al. 1999). This indicates that this flood duration has an effect even in the middle and high marsh. Additionally, the effect of wave forcing is also most likely to be strongest in the pioneer zone, and competition is known to be an important process throughout salt marshes (Bertness and Ellison 1987, Bertness and Hacker 1994, Emery et al. 2001). Therefore, it seems unlikely that the large influence of flood duration is only limited to the pioneer zone. However, further study will have to establish its importance in other salt marsh regions, and with species other than Spartina. Another consequence of limiting ourselves to the pioneer zone is that we reduce the effects of competition, as competition is likely to have strong effects near the border between vegetation types. These borders were excluded as they presented mixed pixels that could not be inverted by the model. We therefore recommend future studies into modeling all salt marsh vegetation types in a single model, in order to expand research beyond the pioneer zone and establish the most important spatial drivers for the entire salt marsh.

Our study site is relatively sheltered. In a more exposed site, the balance between wave forcing and flood duration might shift. It seems unlikely that this would affect the relative importance of the other considered drivers (i.e. competition and creeks). In the sheltered pioneer zone, which is relatively important with regard to the ecosystem service provision (Feagin et al. 2010), it was found that flood duration is the most important driver behind spatial patterns. 


\subsubsection{ProSail}

To establish how flood duration affects reflectance, we used the physical model ProSail. This model combines information on leaf and canopy levels to simulate reflectance spectra. The performed analysis showed that vegetation density and cover, represented by LAI, is the most influential in the reflectance spectra. Therefore, the drivers seem to produce large scale spatial patterns by affecting local values of LAI. LAI is one of the main driving variables and can affect the entire spectrum (Berger et al. 2018), however measuring LAl can be challenging (Zheng and Moskal 2009). A recent global review of ProSail showed that ProSail is often used to model LAI, however there are several problems associated with it (Berger et al. 2018). The inversion of LAI can strongly depend upon the number of satellite bands used in agricultural situations (Verger et al. 2011) and can saturate at higher LAl values (Verger et al. 2011, Berger et al. 2018). This saturation occurred around LAI values of 6 (Verger et al. 2011), which are unlikely to ever occur in European salt marshes. Another problem associated with ProSail in agricultural areas is that at earliest and late growth stages with very low LAI, the background reflectance dominates the spectral signal (Berger et al. 2018). This is reported at the earliest growth stages (Berger et al. 2018), As the measured LAl and the vegetation cover show the soil is not completely covered hence soil reflectance does contribute to the reflectance spectra. The LAl estimate could be improved by including in situ soil spectra in the model.

In this study LAI was estimated with an inversion of the ProSail model, and compared with in situ LAI and cover measurements. This showed a significant relation between modelled and measured LAI and cover, but also indicated the inversion contains significant noise and has a relatively high RMSE.

We found a strong correlation between NDVI and our LAI estimate. NDVI is one of the most often used methods of describing spatial variation in vegetation. The high correlation with NDVI shows that the inverted LAI represent the spatial patterns well. We would caution against using this LAI inversion in an application where the absolute LAI values are of great importance.

This analysis was performed with RapidEye, which has a multispectral satellite sensor with five spectral bands. This satellite constellation is often used for vegetation studies, however the estimation of Leaf Area Index might be improved by using a satellite with more spectral bands. A neural networking approach showed that for LAl estimations 7 bands is optimal (Verger et al. 2011). A better LAl estimate may be obtained with a satellite sensor with both a better spectral and radiometric resolution. RapidEye has a 12-bit digitization with low readout noise 
(Tyc et al. 2005). Its dynamic range of 12 bits/pixel is comparable to other broadband sensors with a similar spatial resolution, such as Pleiades and SPOT 6/7, but is worse than, for example, KompSat and Sentinel-2 (Sozzi et al. n.d.).

On a large spatial scale the effects of leaf properties (chlorophyll $a+b$, carotene) could not be detected. The canopy properties (leaf area index and dry/wet soil fraction) largely overwrite the effects of the leaf properties.

ProSail is mainly used in agricultural settings (Botha et al. 2007, Tripathi et al. 2009, 2012, Si et al. 2012, Duan et al. 2014, Berger et al. 2018), often used to study chlorophyll (Botha et al. 2007, Li et al. 2015, Kooistra and Clevers 2016). We used ProSail to simulate the effect of chlorophyll on reflectance at the leaf scale and found that the range of chlorophyll contents among the leaves observed in the study site was not enough to explain large-scale variations in reflectance, suggesting that chlorophyll content of the leaves does not play a major role in large scale spatial patterns in the pioneer zone of salt marshes. It is likely that a wider range of values (e.g., in chlorophyll content of the leaves) will occur when multiple seasons are measured. However, we only studied a single species during a single season (i.e. Spartina anglica during mid spring). As a consequence, the chlorophyll content at the leaf level had very limited variability and so it had a limited effect on reflectance.

In Spartina stands, our analyses showed that LAI estimates are closely related to NDVI values, agreeing with previous literature (Kearney et al. 2009). This indicates that correlation studies relating biophysical properties directly to a vegetation index are not necessarily improved by using a more complex physical model. In our study, the physical model (ProSail) performed only marginally better than a regular NDVI. The main advantage of using ProSail is that it allows for the distinction between leaf and canopy level effects, and even distinguishes between individual effects. However, for further studies not requiring this level of differentiation, we recommend using simple correlation based on vegetation indices. The slight improvement in performance and understanding is outweighed by the large number of extra input parameters and computation required. 


\subsubsection{Effect of spatial drivers on leaf and canopy level}

In large scale patterns, leaf level biophysics are overwritten by canopy characteristics. However, the leaf characteristics are affected by spatial drivers. Both chlorophyll $a+b$ and carotene contents were strongly affected by the duration of flooding and the influence of creeks. These relations were positive; higher flood duration increases chlorophyll content. As plants that are flooded more often suffer a higher stress, the opposite might be also expected. Decreasing chlorophyll content is a common response to a variety of stressors (Carter and Knapp 2001a). Spartina is also known to decrease its chlorophyll when stressed, but the force required to stress a plant differs greatly between Spartina species (Castillo et al. 2000). It is possible that in our study site Spartina was not stressed by the regular flooding. Plants are able to adapt well to regularly occurring phenomena, such as the day night cycle, and these factors are not considered stressors but are described as 'regular acclimation' (Lichtenthaler 1996). However, our results indicate that biomass is lower when they are flooded more frequently, indicating that they are indeed stressed. This is also indicated by their improved growth when moved higher in the marsh (Bertness and Ellison 1987). Another possibility is that the increase in environmental stress reduces light competition, although our data showed that chlorophyll content is not related to competition. Light availability is also codetermined by flood duration, as top leafs are flooded less than the rest of the plant. We tested the chlorophyll content of fully grown leaves near the top of the plant, so it is also possible that these plants allocate more of their chlorophyll production to their highest leaves, as these are flooded least. This will have to be studied further.

Creeks were also found to strongly affect chlorophyll content ( $a+b$ and carotene). Creeks are known to affect soil conditions (e.g. carbon and nitrogen content) (Zhao et al. 2016), which could play a role in chlorophyll development. However, many of our plots were $>40$ meters away from a creek, and chlorophyll content is lowest closer to a creek. Chlorophyll content is therefore unlikely to be a largely affected by nutrients provided by creeks. It remains unclear how this spatial driver affects vegetation properties. The position of a creek might be codetermined by an underlying cause also affecting chlorophyll content, or the spatial influence of creeks might be larger than expected. 
At the canopy level, we found that LAI and soil moisture content are affected most by wave forcing and competition. In our study area, wave forcing and competition complement each other: high competition only occurs at low wave forcing and vice versa. The Spartina LAl values here presented decreased as competition increased and, correspondingly, also decreased at low wave force levels. A similar pattern is seen in the relation between LAI and flood duration. At low flood duration LAI is low, which corresponds to an increased competition. The highest LAI values occur at intermediate flood duration. This hints towards an optimal growing position, close to the water a high flood duration causes stress, high in the marsh competitors cause stress. This pattern is known in salt marshes (Bertness and Ellison 1987).

Overall, the effects of spatial drivers are stronger at the leaf level; likely because here biophysical properties are relatively simple and easy to measure. At canopy level there is more noise and the effect of spatial drivers becomes less obvious. This is likely because the canopy level has more complex parameters, such as leaf orientation, which are difficult to measure correctly (Berger et al. 2018). The larger variation at canopy level is reinforced by the inherit increase in variation that comes with scaling up. The relationships described by the regression models is intended to show how vegetation characteristics respond to spatial drivers, we would therefore argue for great caution when applying them as predictive models. 


\subsection{Conclusions}

As a first step towards understanding drivers behind spatial patterns in salt marshes we studied the monospecific (Spartina) pioneer zone of a European salt marsh in spring. We found that the spatial patterns where mainly caused by flood duration, which affects spatial patterns through leaf area index. Flood duration explained over three times as much variation as wave forcing, competition or creek influence. The influence of drivers on spatial patterns seems to be stronger on canopy properties, especially leaf area index, than on leaf characteristics, which play only a minor role. This knowledge is a first step towards improving our capacity to use remote sensing signals as proxies for salt marsh mechanisms. Since simple indices such as NDVI performed nearly as well as physical models in our salt marsh pioneer area, NDVI may be well suited for monitoring these relatively simple systems.

\section{Author Contributions:}

Conceptualization, B. Oteman, E.P. Morris and D. van der Wal;

Data curation, B. Oteman and E.P. Morris;

Formal analysis, B. Oteman and E.P. Morris;

Funding acquisition, G. Peralta and D. van der Wal;

Investigation, B. Oteman and E.P. Morris;

Methodology, B. Oteman, E.P. Morris and D. van der Wal;

Project administration, G. Peralta and D. van der Wal;

Resources, B. Oteman, E.P. Morris and D. van der Wal;

Software, B. Oteman and E.P. Morris;

Supervision, T.J. Bouma and D. van der Wal;

Validation, B. Oteman and E.P. Morris;

Visualization, B. Oteman;

Writing - original draft, B. Oteman, E.P. Morris and D. van der Wal; Writing review \& editing, B. Oteman, G. Peralta, T.J. Bouma and D. van der Wal. 


\title{
Chapter 4: Indicators of expansion and retreat of Phragmites based on optical and radar satellite remote sensing: a case study on the Danube delta
}

\section{Authors:}

B. Oteman ${ }^{1}$, A. Scrieciu ${ }^{2}$, T.J. Bouma ${ }^{1}$, A. Stanica ${ }^{2}$, D. van der Wal ${ }^{1,3}$

1 NIOZ Royal Netherlands Institute for Sea Research, Department of Estuarine and Delta Systems, and Utrecht University, P.O. Box 140, 4400 AC Yerseke, the Netherlands.

2 GeoEcoMar Romanian National Institute for Research and Development for Marine Geology and Geo-ecology, Str. Dimitrie Onciul 23-25, sector 2, 024053 , Bucharest, Romania

3 Faculty of Geo-Information Science and Earth Observation (ITC), University of Twente, P.O. Box 217, 7500 AE Enschede, The Netherlands.

*correspondence: bas.oteman@nioz.nl, TEL: +31 (0)113 577300

\begin{abstract}
Reed is an important wetland species, as on the one hand it provides valuable ecosystem services at some places, while on the other hand it poses a threat as invasive species in other places. Thus monitoring and predicting reed dynamics is crucial for wetland managers. Hence, we developed an indicator for the stability of reed wetlands based on remote sensing technology. We used Landsat satellite imagery to study reed development in the Razelm Sinoe Lagoon System, part of the Danube Delta in Romania over a period of 22 years, and compared the development (expansion, stable or lateral loss) of reeds with optical and radar information. In our study area, there were clear spatial differences in long-term reed dynamics, with reed expansion being most likely in relatively sheltered locations. Comparing optical and radar data from the Sentinel satellite constellation revealed a clear difference in radar (VV and VH C-band SAR) backscatter, but no difference in the optical (NDVI, SAVI) signal of expanding, stable and decreasing reed areas. The radar data showed the largest seasonal differences in backscatter signal in locations where reed was expanding and smallest seasonal differences in areas where reed was decreasing.

Overall, our study shows that the stability of reed ecosystems, and their corresponding services, can be monitored by quantifying seasonal changes in backscatter of reed lands using radar satellites. This principle looks promising for monitoring other ecosystems as well.
\end{abstract}




\section{Keywords}

Reed, reed development, marsh, wetland, remote sensing, radar, Danube delta

\subsection{Introduction}

Wetland ecosystems provide valuable services. For example, they provide habitats for unique plant species and animal communities which may in part serve as food (Henderson and Lewis 2008, de Groot et al. 2011). They also provide economic benefits (Henderson and Lewis 2008, Chmura 2011) and a nursery for fish, thereby supporting local fisheries (Boesch and Turner 1984, Deegan et al. 2002, Chmura 2011). In addition, wetlands may reduce nutrient loading in the coastal waters (Chmura 2011) and serve to mitigate waves and reduce flood risk (Möller 2006, Barbier et al. 2008, Koch et al. 2009, Morgan et al. 2009, Chmura 2011, de Groot et al. 2011). Monitoring the stability and edge dynamics of wetlands, and thereby the ecosystem services they provide, is thus important to enable suitable management.

Here, we focus on the stability of reed wetlands. Reed (Phragmites australis) is a common plant in wetlands and is well known for its phytoremediation (Guo et al. 2014), but is also recognized as an important species for shoreline protection, and protection of arthropods, birds and mammals (Ostendorp et al. 2003, Cerri et al. 2017). Reed also provides raw materials, and can provide food for livestock (Stanica et al. 2012). The function of reed as a buffer between aquatic and terrestrial ecosystems makes reed one of the most important wetland species (Brix 1999). Since the 1950's, reed has shown a severe die-back in eastern, central and northern Europe. This phenomenon was named the Reed Die-Back Syndrome (RDBS). The warmer Mediterranean region did not seem affected, and reed here even seemed to expand rapidly (Van Der Putten 1997). However, more recently reed die-back was also detected in Italy (Fogli et al. 2002, Cerri et al. 2017).

In contrast to the reed die-back in northern Europe, in the United States reed is rapidly expanding and commonly treated as an unwanted invasive species, reed eradication programs are a common part of management (Chambers et al. 1999, Silliman and Bertness 2004). Recently it has been suggested that the European subspecies of reed is outcompeting the North American subspecies, with large potential effects for the ecosystem, as reed can have a large effect on the nitrogen balance of a system (Findlay et al. 2002, Volesky et al. 2018). Rapid expansion of reed lands in North America could indicate replacement by the European subspecies. 
Many possible causes of reed die-back or reed expansion have been suggested (Van Der Putten 1997, Fogli et al. 2002, Cerri et al. 2017), but as of yet it is unclear which set of conditions are linked with these developments. Hence, we lack reliable methods to predict where die-back or expansion may occur. Remote sensing is a promising technique for both monitoring long-term reed dynamics as well as obtaining indicators for predicting future reed development.

In this study we aim to take the first steps towards developing remote sensing techniques to both monitor and predict future reed development. We will do this for the Danube delta along the Black Sea coast in South Eastern Europe, one of the worlds' largest area naturally covered by wetlands. Here, reeds have been continuously harvested for centuries and local communities greatly depend upon their many ecosystem services (Stanica et al. 2012). The Razelm Sinoe Lagoon System, in the Danube Delta in Romania was chosen as study area. This lagoon system at the end of the Danube river, along the Black Sea, provides a massive coastline covered almost exclusively by reed, which is very important for local communities (Stanica et al. 2012). It is unclear if the Danube delta follows the southern European trend of rapid expansion, or if there are signs of vegetation dieback like sites in Italy (Fogli et al. 2002, Cerri et al. 2017) and Hungary (Van Der Putten 1997).

To effectively monitor the vast area of the Razelm Sinoe Lagoon System $\left( \pm 1000 \mathrm{~km}^{2}\right)$, we use space borne remote sensing. Several studies have already looked into detecting reed using remote sensing to support management; reed and other vegetation can be distinguished using optical (Pengra et al. 2007) or radar data (Bourgeau-Chavez et al. 2013). In the south of France, an extensive model was used to model reed height, diameter, flower head density, stem density and cover based on six optical satellite (SPOT 5) images covering a single growing season (Poulin et al. 2010). They found that although the Normalized Difference Vegetation Index (NDVI) performed poorly, the Soil Adjusted Vegetation Index (SAVI) worked well. Space borne X-band radar has been applied to differentiate between reed, Suaeda japonica, and mudflat (Lee et al. 2012). C-band radar can also be used to classify wetlands (Baghdadi et al. 2001) and Sentinel 1's C-band radar is expected to be very useful for wetland analyses (Reschke et al. 2012). A study (Brisco et al. 2011) using airborne C-band radar found that reed produces backscatter patterns similar to other grasses. Following the Freeman-Durden model (Freeman and Durden 1998), they distinguished between double bounce scattering, volume scattering and surface scattering and considered Phragmites a volume scattering object (Brisco et al. 2011). In other words, they expect most of the signal to be scattered by the vegetation layer. Both optical and radar remote 
sensing have been applied with success to reed monitoring before (Poulin et al. 2010, Lee et al. 2012).

In this study, we will use a large historical optical satellite data set (the Landsat archive) to establish reed development over a period of 22 years. We subsequently aimed to detect differences in the state of reed areas that were expansion, stable or showed lateral loss. To do so, we compare the optical (Sentinel $2 \mathrm{MSI}$ ) and radar (Sentinel $1 \mathrm{C}$-band SAR) signals, of increasing (lateral expansion), decreasing (lateral loss) and stable reed areas. Additionally we investigate the effect of the fetch (the length of the open area the wind and waves have to build), as it has been suggested to influence expansion (Coops et al. 1994) and we establish the effect of seasonal development patterns, as a stressed system might have a smaller lateral expansion of reed in spring.

Summarizing, the overarching aim of this study is to establish an indicator to help monitor reed-dynamics, and hence the stability of the ecosystem services the reed provides. We do so by focusing on indicators that can be derived from satellite remote sensing data, and that distinguish between areas with die-back versus expansion. We subsequently apply this indicator to a major coastal reed land in the Razelm Sinoe Lagoon System in Romania, to establish if reed wetlands are dying back or expanding. This will directly support monitoring efforts in Romania, but might also be a first step towards mapping reed die-back or expansion in other areas.

\subsection{Materials and methods}

To gain insight into reed development in the Danube delta, we first establish where expansion and retraction has taken place, by comparing two images from the Landsat archive, 22 years apart. Then, we use recent optical (Sentinel $2 \mathrm{MSI}$ ) and radar (Sentinel 1 SAR) satellite images to compare expanding, stable and eroding areas in winter, spring and summer. In situ measurements in the Razelm Sinoe Lagoon System were taken to establish the underlying physical characteristics of the reed beds. 


\subsubsection{Area description}

The primary study site is the Razelm Sinoe Lagoon System, in Romania. This is the southern part of the Danube Delta Biosphere Reserve. The entire system covers about $1000 \mathrm{~km}^{2}$, and consists of a series of lakes, former lagoons transformed into lakes and present-day lagoons with engineered inlets, as well as other ponds, wetlands, former coastal sandbars, fossil and present day littoral barrier beaches. The coastal vegetation consists mainly of reed (Phragmites australis) which can grow up to six meters, before dying in winter (Hanganu et al. 2002). The lagoon has seen major human influences the last century. The connection with the nearby fresh water system, the Danube, was increased by cutting new canals and dredging existing channels, followed by closing down of the main inlet in 1974 (at Gura Portitei) and replacement of the natural connections with the sea with engineered inlets (for Sinoe Lagoon). This caused the system to move towards a fresh water system. This also meant an increase of Danube-born nutrient and sediment inflow in the lagoon system. The limited connection with the sea causes the system to accumulate both organic and non-organic matter (Stanica et al. 2012, Dinu et al. 2015). The climate of the Lagoon System is continental, with hot dry summers and very cold winters. The Lagoon System is located in one of the windiest areas in Romania. The water bodies of the lagoon system are partly or entirely frozen during winter, the freezing periods vary from days to weeks, but complete ice cover for long periods is rare.

\subsubsection{Seasonal in situ measurements of reed characteristics}

At two locations in the Razelm Sinoe Lagoon System, in situ measurements were taken, the first site, Jurilovca, is located at lat: $44.75^{\circ}$, lon: $28.94^{\circ}$, the second site, Histria, is located at lat: $44.55^{\circ}$, lon: $28.78^{\circ}$. Site Jurilovca was sampled in March, May and July 2015. Site Histria was sampled in the same months in 2016 (see appendix 4.1 for a detailed map of both sites). In each location, five destructive plots and six non-destructive plots of $1 \times 1 \mathrm{~m}$ are placed, their position was recorded with a GPS. In each plot, the number of reed stems was counted by placing a $0.5 \times 0.5$ meter frame and counting all stems with a length $>30 \mathrm{~cm}$, stems with a shorter length were ignored. In each plot, 20 representative stems are selected, their diameter is measured approximately 10 centimeter above the ground. These stems were harvested and their length was recorded. They were weighed, dried for $>3$ days at $60^{\circ}$ and reweighed to establish wet and dry biomass. 


\subsubsection{Categorizing long-term reed development from Landsat}

\section{imagery}

To establish vegetation development in the Razelm Sinoe Lagoon System we used two Landsat images 22 years and 4 days apart (Landsat 5 TM at 1995-07-04 and Landsat $8 \mathrm{OLI}$ at 2017-06-30). We used the surface reflection images provided by the USGS, made available through EarthExplorer. The rough outlines of the lagoon were manually drawn, to ensure we only have vegetation and water in the area of interest and all roads and other human structures are excluded. Within these limits, a threshold of 0.3 in the Normalized Difference Vegetation Index NDVI was applied to distinguish between land and water, which was visually checked and performed well. In the Razelm Sinoe Lagoon System, a small section at the southern tip of Sinoe lake was removed from analyses, as it proved difficult to distinguish between water and vegetation here, likely due to a mix of dead vegetation, water and sediment. This small section was not taken into consideration for any of the consecutive analyses. In addition, some areas were excluded due to clouds and cloud shadows in the imagery. Appendix 4.2 provides the outline of the applied masks.

The water edge of 2017 was used to extract a strip of $60 \mathrm{~m}$ of vegetation. For pixels in this strip, the lateral development of the reeds (stable, increase or decrease) were established. Within this strip, values were extracted every $30 \mathrm{~m}$, conform the spatial resolution of the Landsat imagery. The distance to the water was calculated for each pixel in each image; this distance was used to distinguish between increasing, stable and decreasing pixels. A pixel with an unchanged distance to water or a pixel where this distance is larger than 45 meters in both 1995 and 2017 was classified as stable (see figure 4.1). The remaining pixels were divided as: decrease $<0<$ increased. Note that the pixels that were in the area with decreased reeds were still vegetated (as by definition pixels with vegetation in 2017 were selected). We then used the Sentinel satellite data, available only for recent years, to look at vegetation properties in retreating, stable and expanding reeds. 


\subsubsection{Establishing wave exposure of reeds, using fetch length}

\section{from Landsat imagery}

Based on the established vegetation edge for the Landsat 8 image of 2017-06-30, the so-called fetch was calculated, indicating the length over which wind-waves can build up over water. To establish the fetch, we determined the angle towards the water using the direction raster of the Euclidean Distance tool in ArcGIS. The fetch was then calculated as the distance in that direction until land (on the opposite side of the lagoon) was reached. Given the jagged shoreline, land closer than $100 \mathrm{~m}$ was not considered as the opposite side of the lagoon, and was ignored. Note that in this study, fetch was calculated for the onshore direction only (i.e., perpendicular to the coastline), and it is thus independent of actual wind direction. For each of the reed development categories (increase, stable, decrease) the mean fetch length was established to test whether shoreline change depended on the exposure of the reed to wind-waves.

\subsubsection{Establishing long-term reed development from Landsat}

\section{imagery}

To gain further insight into the long-term reed development, we analyzed six additional Landsat images (of: 1999, 2001, 2005, 2008, 2011 and 2015, see table 4.1). We processed these similar to the previous Landsat images and calculated the distance to the water for each sampling point. We tested the performance of our manual threshold of NDVI by applying the ArcGIS tool 'Iso Cluster Unsupervised Classification' (using 2 classes) to the NDVI. This consistently estimated the land water threshold at an NDVI between 0.25 and 0.3 . We used the unsupervised clustering threshold analyses to distinguish between land and water. We used images from the same season (June/July) and used a compounding cloud mask (i.e. pixels that were covered by clouds or cloud shadows in any year were completely removed from the analysis). For the pixels in the selected strip along the shoreline, we then analyzed the position of the pixels relative to the shoreline, for the three reed development categories (retreat, stable and expansion). We used the development categories established between 1995-2017 to compare the distance to the water edge between categories. This provided insight into the persistence of the reed development.

Table 4.1, Landsat images of the Razelm Sinoe Lagoon System for multi-year analysis. 


\begin{tabular}{ll}
\hline Satellite & Date \\
\hline Landsat 5 & $1999-06-29$ \\
Landsat 5 & $2001-07-04$ \\
Landsat 5 & $2005-06-29$ \\
Landsat 5 & $2008-07-07$ \\
Landsat 5 & $2011-07-16$ \\
Landsat 8 & $2015-07-11$ \\
\hline
\end{tabular}

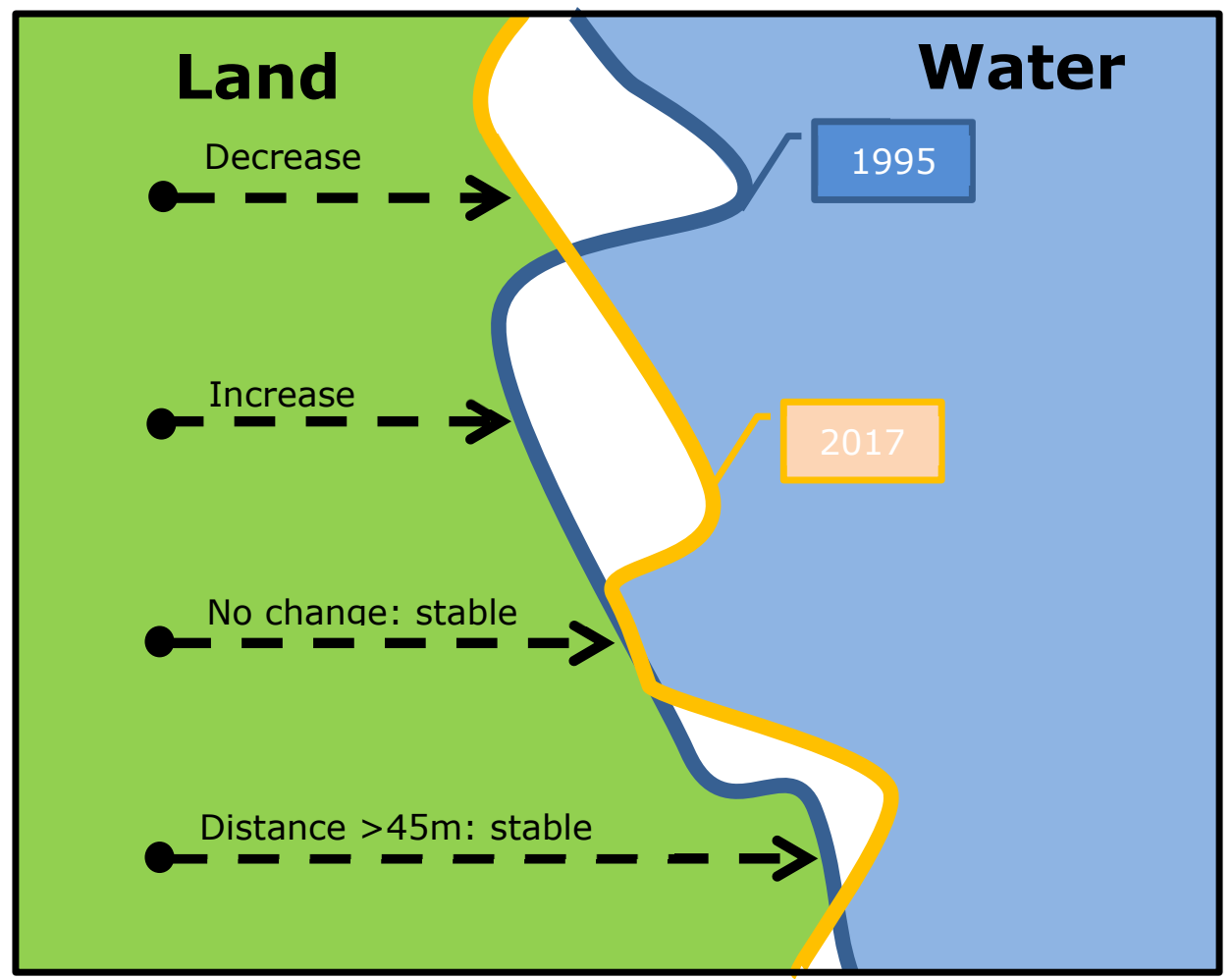

Figure 4.1: Schematization of assigning the reed development categories (decrease, stable, increase) based on shoreline changes between 1995 and 2017. 


\subsubsection{Analyzing seasonal remote sensing indicators of long-}

\section{term reed development}

The Copernicus Sentinel satellite constellation (including, among other sensors, Sentinel-1 SAR and Sentinel-2 MSI) is used as primary data source to examine how vegetation development could be observed remotely. All preprocessing was done with the SNAP toolbox (v5.0) provided by the European Space Agency. Sentinel-1 SAR multi-look, dual polarized (VV and VH) level-1 Ground Range Detected (GRD) interferometric wide swath (SM) images were preprocessed by first removing thermal noise, followed by the application of an orbit file. Then data were calibrated to sigma nought, and a range Doppler terrain correction was applied. Sentinel-2 MSI images, with $10 \mathrm{~m}$ resolution, were atmospherically corrected by a dark pixel approach using the Sen2cor tool set in SNAP. We used Sentinel images of winter, spring and summer. The seasonal timing of these images matched the in situ measurements, see table 4.2. A single cloud and cloud shadow was removed from the Sentinel-2 images of 2016-06-07, the cloud stretched over approximately 700 meters of coast. Where the cloud partially or completely covered the reed bed, the entire reed bed perpendicular to the water was removed from the analysis. The average VV backscatter and VH backscatter from Sentinel-1 SAR and average NDVI from Sentinel-2 MSI were compared between increasing, stable and decreasing reed beds.

To analyze seasonal effects, the water line was individually established for each Sentinel-2 MSI image, for the first 2 images (2016-01-09 and 2016-04-28) a NDVI threshold of 0 was used (NDVI $<0$ was assigned water). This threshold performed poorly in the July image, where water had a positive NDVI value, possibly due to phytoplankton. For this image a threshold of 0.3 was used $(N D V I<0.3$ was assumed water). The difference in NDVI between vegetated and unvegetated foreshore is typically distinct, the established thresholds were checked visually and found to perform well. To exclude potential mixels, we only used pixels found to be vegetation in all Sentinel-2 images.

In addition to the NDVI, the Soil Adjusted Vegetation Index (SAVI), with a default $L$ value of 0.5 , was also used as this was found to perform better than NDVI when analyzing reed vegetation (Poulin et al. 2010).

The NDVI, VV and VH backscatter of Sentinel images were compared between reeds established to be declining, stable or expanding (as retrieved from the Landsat imagery). This was done for the Sentinel-1 SAR and Sentinel-2 MSI images of winter, spring and summer, respectively. 
To establish which biophysical properties had a large effect on remote sensing imagery, we compared in situ biophysical measurements with seasonally matching remote sensing images. In situ measurements of biomass, vegetation height, stem diameter and stem density were compared with VV, VH backscatter and NDVI at that location (the values of the corresponding pixel was extracted) using separate linear regressions. The three different seasons were tested separately. Locations of the in situ stations are provided in Appendix 4.1.

Table 4.2, Dates of in situ sampling and corresponding Sentinel-1 SAR (S1) and Sentinel-2 MSI (S2) images of the Razelm Sinoe area.

\begin{tabular}{|c|c|c|c|c|}
\hline $\begin{array}{l}\text { In situ } \\
\text { date }\end{array}$ & Locatic & Season & S1 date & S2 date \\
\hline $2015-3$ & Jurilovca & Winter & $2015-01-12$ & 2016-01-09 \\
\hline $2015-5$ & Jurilovca & Spring & $2015-05-12$ & $2016-05-22$ \\
\hline $2015-7$ & Jurilovca & Summer & $2015-07-23$ & 2016-06-07 \\
\hline $2016-3$ & Histria & Winter & 2015-01-12 & 2016-01-09 \\
\hline $2016-5$ & Histria & Spring & $2015-05-12$ & $2016-05-22$ \\
\hline $2016-7$ & Histria & Summer & $2015-07-23$ & 2016-06-07 \\
\hline
\end{tabular}

\subsection{Results}

\subsubsection{Long-term reed development from remote sensing}

From the Landsat optical data of 1995 and 2017, we found a vegetation loss of 38 ha (426 pixels), whereas the vegetation was found to be stable in 265 ha (2944 pixels) and increased (expanded) in 1946 ha (21627 pixels) in a period of 22 years. The multi-year analysis showed a strong linear increase in locations labelled as expanding reed (see figure 4.2). There was an overall decrease in reeds in locations marked as such; the rate of decrease was especially large in the period 2008-2011. The water distance of stable areas also increased, likely because points where the water distance was larger than $45 \mathrm{~m}$ were also labelled as stable. 


\subsubsection{Seasonal remote sensing as indicators of long-term reed development}

The Sentinel-2 optical data showed that the NDVI was lowest in winter, increases in spring and was highest in summer. Although the NDVI seemed higher in stable and increasing sites in summer, the differences between decreasing, stable and increasing reeds were small (figure 4.3). SAVI showed the same pattern as NDVI, with clear differences between seasons, and small differences between eroding, stable and expanding reed sites. Decreasing, stable and increasing sites could not be distinguished using optical satellite data (NDVI or SAVI).

The Sentinel-1 SAR data showed low values for the backscatter, and hence likely smooth vegetation in winter, relatively rough vegetation in spring and an intermediate roughness in summer. Interestingly, in sites with decreasing reed beds, the vegetation gave a smooth backscatter, the vegetation was rougher in stable reed beds and the roughest vegetation structure was found in increasing reed beds. This pattern (i.e., a strong contrast in backscatter between the different reed development categories) was especially clear in spring, but was also visible in summer. The winter backscatter was similar between decreasing, stable and increasing sites, but in spring large differences had developed (see figure 4.3). VV and $\mathrm{VH}$ polarized radar showed a similar pattern, the largest seasonal changes were found in the expanding reed beds, whereas the decreasing reed beds showed least seasonal changes.

Established vegetation appeared to be able to persist in areas with a long fetch, but an increase (reed expansion) mainly occurred in relatively sheltered locations (see figure 4.4). Areas which had a decrease in reed beds, had on average an intermediate fetch but with a large spread, suggesting that die-back of reed can occur regardless of fetch length. These decreasing sites also showed limited seasonal expansion, but with large variation. Increasing and stable sites showed a larger seasonal expansion (see figure 4.5).

\subsubsection{Relating seasonal remote sensing indicators to in situ}

\section{patterns}

We compared the in situ measurements with radar backscatter to explain which vegetation properties cause patterns in backscatter, and hence which underlying processes can explain the seasonal differences in backscatter. However, the in situ measurements showed no clear relation with backscatter. 
We tested 36 hypotheses simultaneously, hence according to the Bonferroni correction our $p$-value should be $<0.0014$ to be statistically significant, none of the tested variables met this threshold (see table 4.3). The in situ data did not show a strong effect on backscatter. We can therefore not definitively conclude what property or process underlies the seasonal changes in backscatter. However, differences in thickness of the vegetation layer (vegetation height) and properties of the vegetation layer (such as leaf area index) would likely have caused differences in NDVI, which was found to be similar between increasing, decreasing and stable sites. The same can be said for mixels containing both vegetation and standing water, as this would not only have lowered the backscatter, but also have lowered the NDVI between expanding and retracting reed beds.

\subsubsection{Seasonal variation in properties of reed vegetation measured in situ}

The in situ data of both field sites show a slow reed development in spring. Biomass seemed lowest in spring, possibly indicating that the vegetation had only just started to develop. However, biomass was not significant different among seasons. The stem diameter was highest in spring, this might be due to a quicker disappearance of smaller stems, although stem density was not significantly different between seasons. Only vegetation height was significantly higher in winter, indicating that dead stems were still present in winter. All vegetation properties measured in situ showed great variation (see figure 4.6). 
Table 4.3, the seasonal relation between in situ measurements and remote sensing signals.

\begin{tabular}{|c|c|c|c|c|c|c|c|c|c|c|c|}
\hline Season & $\begin{array}{l}\text { Biophysical } \\
\text { properties }\end{array}$ & $n$ & NDVI & $\mathrm{P}$ & R2adj & $w$ & $\mathrm{P}$ & $\mathrm{R} 2 \mathrm{adj}$ & $\begin{array}{l}\mathrm{VH} \\
\mathrm{F} \\
\end{array}$ & $\mathrm{P}$ & R2adj \\
\hline Spring & Biomass & 9 & 1.547 & 0.254 & 0.064 & 0.010 & 0.925 & -0.141 & 1.471 & 0.265 & 0.056 \\
\hline Spring & Density & 14 & 0.564 & 0.467 & -0.035 & 0.120 & 0.735 & -0.073 & 1.494 & 0.245 & 0.037 \\
\hline Spring & Diameter & 15 & 2.146 & 0.167 & 0.076 & 2.973 & 0.108 & 0.124 & 0.769 & 0.396 & -0.017 \\
\hline Spring & Height & 9 & 2.613 & 0.150 & 0.168 & 0.021 & 0.889 & -0.139 & 0.013 & 0.914 & -0.141 \\
\hline Summer & Biomass & 15 & 1.468 & 0.247 & 0.032 & 0.003 & 0.960 & -0.077 & 0.182 & 0.677 & -0.062 \\
\hline Summer & Density & 30 & 0.021 & 0.887 & -0.035 & 1.309 & 0.262 & 0.011 & 0.336 & 0.567 & -0.023 \\
\hline Summer & Diameter & 37 & 0.067 & 0.797 & -0.027 & 0.349 & 0.558 & -0.018 & 0.151 & 0.700 & -0.024 \\
\hline Summer & Height & 15 & 0.865 & 0.369 & -0.010 & 0.384 & 0.546 & -0.046 & 2.455 & 0.141 & 0.094 \\
\hline Winter & Biomass & 17 & 1.156 & 0.299 & 0.010 & 1.740 & 0.207 & 0.044 & 0.184 & 0.674 & -0.057 \\
\hline Winter & Density & 23 & 0.003 & 0.955 & -0.047 & 0.491 & 0.491 & -0.022 & 1.544 & 0.227 & 0.022 \\
\hline Winter & Diameter & 29 & 0.025 & 0.877 & -0.036 & 2.175 & 0.152 & 0.040 & 0.109 & 0.744 & -0.033 \\
\hline Winter & Height & 18 & 0.436 & 0.518 & -0.034 & 0.192 & 0.667 & -0.050 & 0.046 & 0.836 & -0.119 \\
\hline
\end{tabular}


Figure 4.2, The multi-year development of locations with decreasing, stable and increasing reed vegetation. This figure shows the development of the average distance to the water (shoreline) of the different development classes (decrease $n=631$, stable $n=4064$ and increase $n=16108$ ) based on Landsat images. The development class was determined over the entire period (1995-2017).

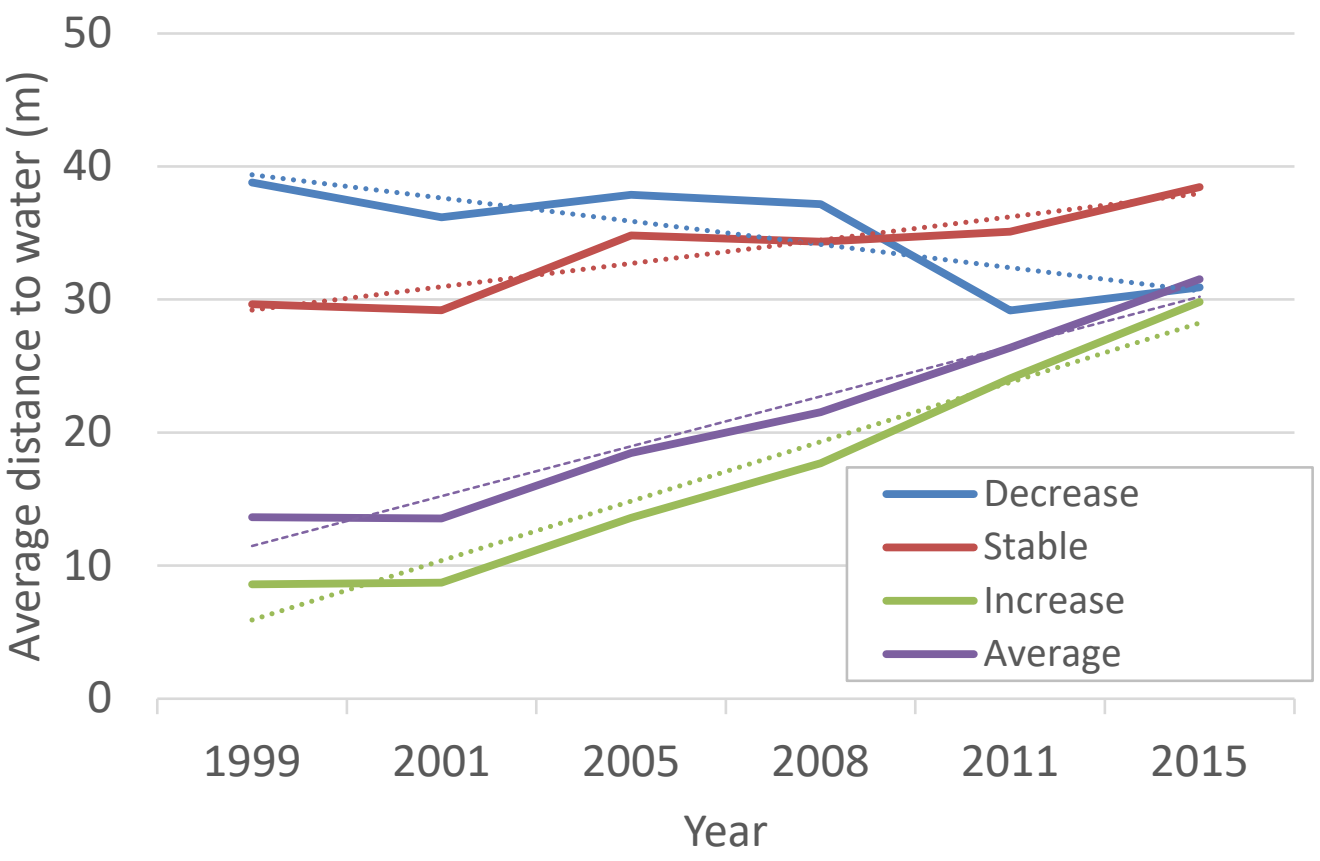


Figure 4.3, Seasonal patterns in optical and radar signals of decreasing, increasing and stable sites, in the entire Razelm Sinoe Lagoon System.

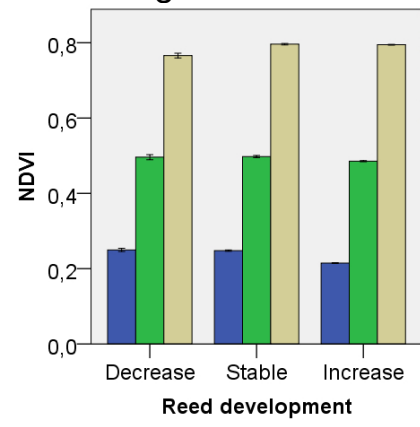

NDVI winter

$$
\begin{aligned}
& \square_{\text {spring }}^{\text {NDVI }} \\
& \hline \text { NDVI } \\
& \hline \text { summer }
\end{aligned}
$$
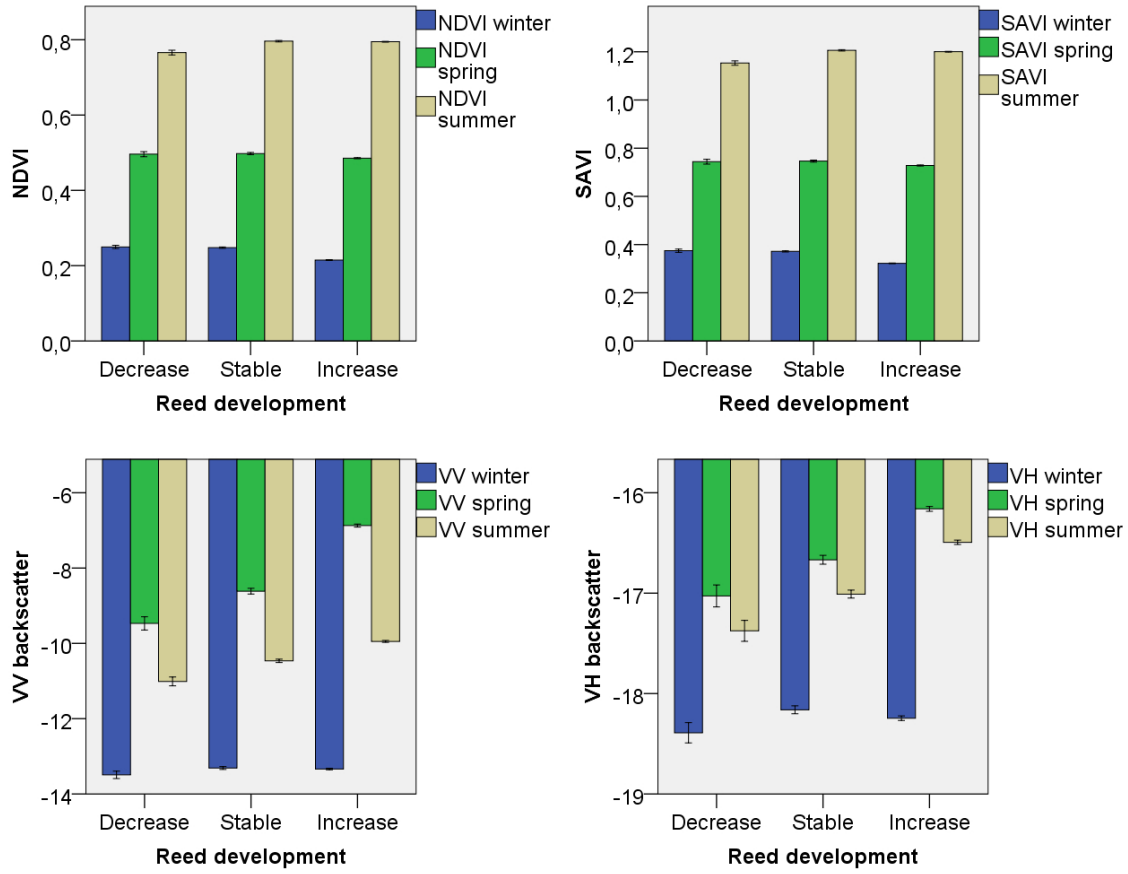
Chapter 4: Indicators of expansion and retreat of Phragmites based on optical and radar satellite remote sensing: a case study on the Danube delta

Figure 4.4, the fetch (length of open water in front of vegetation) in decreasing, increasing and stable locations.

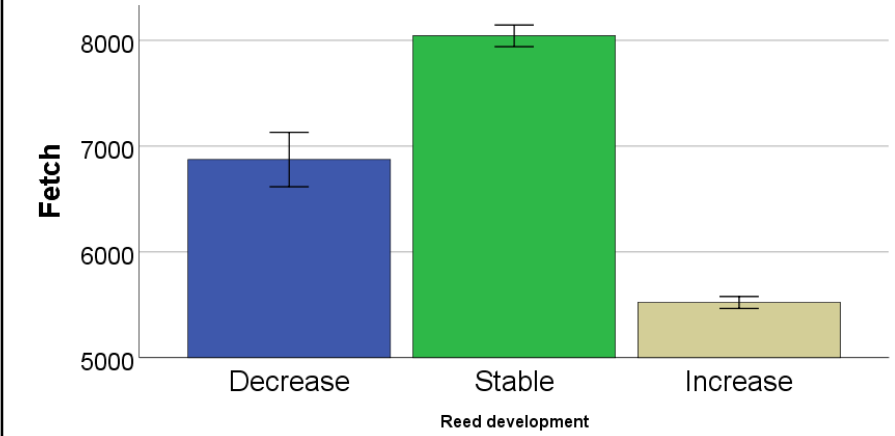

Figure 4.5, the seasonal expansion of decreasing, increasing and stable locations.

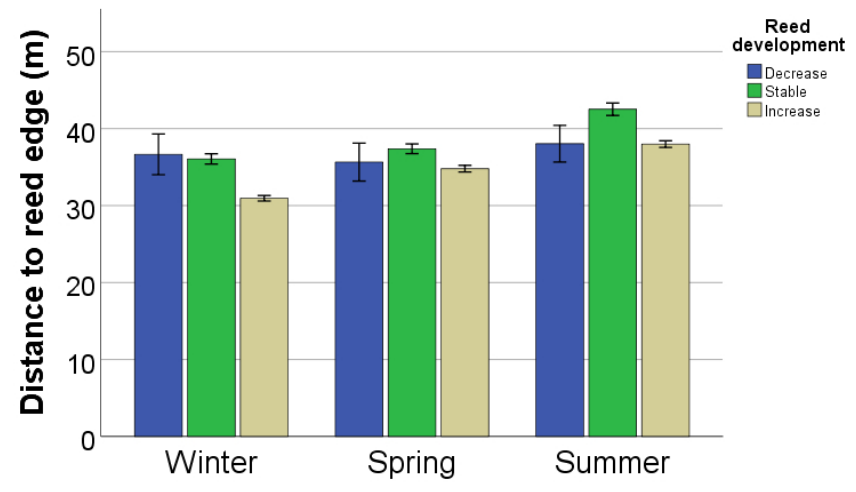


Chapter 4: Indicators of expansion and retreat of Phragmites based on optical and radar satellite remote sensing: a case study on the Danube delta

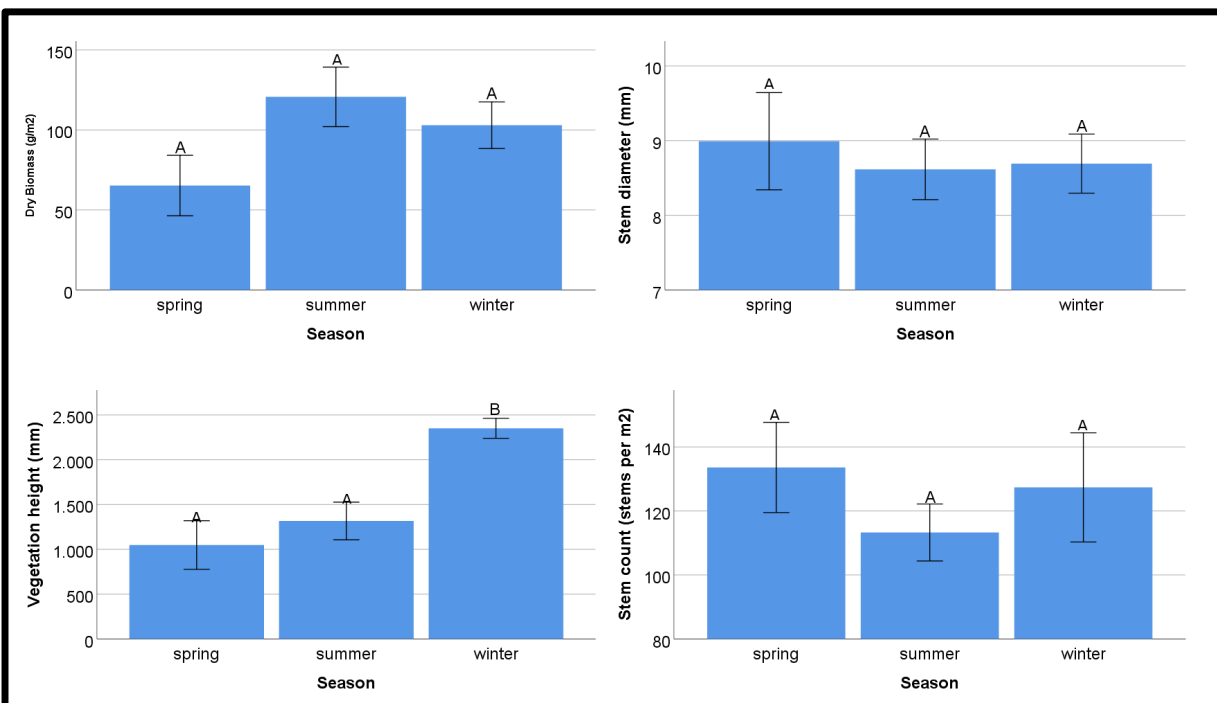

Figure 4.6. Seasonal differences between in situ measured properties of reed vegetation. 


\subsection{Discussion}

The aim of this study was to 1) establish an indicator to enable monitoring of reedwetland dynamics and thereby the ecosystem service stability, and 2) apply it to the Razelm Sinoe Lagoon System in Romania to establish the stability of these reed wetlands. We used optical and radar satellite data to compare increasing, decreasing and stable reed edges of wetlands and found that especially the seasonal differences in radar backscatter provides valuable insight into the longterm vegetation stability. That is, indicators based on optical data showed no difference between the areas, but indicators based on SAR data showed large differences between decreasing, stable and increasing reed lands. The decreasing sites are roughest in spring and summer, stable sites are in between and expanding sites are smoothest. In addition, the differences between winter, spring and summer are largest in expanding sites, intermediate in stable sites and smallest in decreasing sites.

\subsubsection{Seasonal remote sensing as indicators of long-term reed}

\section{development}

We expect that the magnitude of the observed patterns in seasonal development of roughness to be site specific, due to climatological differences and site-specific differences in local environmental conditions. However, the seasonal pattern of a slower development and smaller seasonal differences in retreating sites is expected to be more general. A decrease in development and recovery speed after disturbance at vulnerable sites is also described by the critical slowing down theory (Scheffer et al. 2001). This theory indicates that as resilience decreases, the system takes longer to recover from disturbance. This could explain the limited and lower seasonal expansion at decreasing sites, which would have a lower resilience. However, although the first experimental evidence of this theory in wetlands is starting to emerge (van Belzen et al. 2017), the application of this theory to reed-lands has yet to be tested. This theory also indicates that plants can remain seemingly healthy up to a collapse, and that optical measurements related to biomass (such as NDVI) are ineffective for measuring resilience. In our study, we also found little response to lateral change in the reed beds in the optical data. However it is unclear if the observed differences in radar backscatter are related to a decrease in resilience or caused by another biophysical process. Notably, the large seasonal variation in back-scatter at the expanding site should not be misinterpreted as flickering, which is often regarded as an indicator of nearness of 
collapse (Dakos et al. 2013). The seasonal variation seems to rather indicate growth vigor.

A multi-seasonal approach is known to be important when studying reed vegetation (Poulin et al. 2010, Lee et al. 2012). Hence seasonal changes were expected to hold information on potential lateral increase or decrease of the reed. There was a clear seasonal trend in the lateral extent of the reed bed, with reed beds protruding into the lake in summer. However the seasonal difference in backscatter in the reed bed vegetation between decreasing stable and increasing reed beds has not been described before. The early growing season is known to be very important for reed, as reed particularly depends on its initial growth to establish competitive dominance (Yamasaki and Tange 1981). The differences in backscatter between decreasing, stable and increasing sites could therefore be due to an earlier start of growth at more favorable locations. However, this would likely also create a difference in biomass, which would have been detectable by optical satellites. A previous study also found little use for NDVI in reed vegetation, but SAVI performed well (Poulin et al. 2010). However, in our study SAVI performed very similar to $\mathrm{NDVI}$; neither of them showed a difference in reed vegetation between expanding, retracting and stable sites.

Roughness data, through radar, is able to provide valuable information that we could not derive from optical remote sensing. However it remains unclear what the underlying processes are. Graham and Harris (Graham and Harris 2003) created a water cloud model to simulate radar backscatter. They identified the most important factors in radar backscatter as the thickness of the vegetation layer (the vegetation height), the internal properties of the vegetation layer (leaf area index or leaf properties), the vegetation moisture content and the soil moisture content. We expect that if the seasonal differences in backscatter between increasing and decreasing locations were caused by thickness or the properties of the vegetation layer we would have detected it in the NDVI. Although indices such as NDVI are known to become insensitive at higher values (Baret and Guyot 1991, Zhang et al. 2013), the NDVI did show seasonal differences, indicating it was not saturated in all seasons. However, we cannot rule out properties of the vegetation layer, such as internal leaf structure, that do not affect NDVI but could have affected radar backscatter.

Vegetation and soil moisture content are major contributors in the water cloud model, an often used model to simulate radar backscatter of land based vegetation (Graham and Harris 2003). The additional water layer in case of flooded vegetation is expected to have an even larger effect on backscatter than soil moisture content, 
resulting in a decrease in backscatter, even though the reed vegetation above it is over $2 \mathrm{~m}$. This water layer is likely largely affected by elevation, as reed at lower elevations has to highest chance of being flooded. Retraction and expansion of the reed may also be a function of elevation. However, a large difference in suitability of conditions for reed growth would likely also have been visible in the optical data. Further study, likely combining remote sensing with an extensive field measurement campaign, will be required to establish which biophysical or environmental factors causes the seasonal difference between radar backscatter of decreasing, stable and increasing reed beds.

\subsubsection{Long-term reed development from remote sensing}

Our analysis showed that in the Razelm Sinoe Lagoon System reed is, overall, expanding. We found an average expansion of 87 ha per year between 1995 and 2017, clearly showing that the massive reed die-back found elsewhere in Europe does not extend to the Danube - Black Sea System. Although reed die-back has been found in southern Europe (Fogli et al. 2002, Cerri et al. 2017) our findings support earlier notions that reed is expanding (Van Der Putten 1997). The reed expansion although large in surface area is still relatively small when compared with the area of the entire lagoon system. On average the expansion is about 1.1 meter per year. A study in the United States, where reed is considered to expand rapidly and management is focused on removing reed, found an invasion rate between 0.1-0.7 meter per year, depending on which species it was competing with (Silliman and Bertness 2004). It is important to note that these are invasion and not expansion rates. In this light the expansion rate found in Romania can be considered rapid. However, it should be kept in mind that this long-term analysis was done using the Landsat satellites; the relatively low resolution of this satellite makes precise estimates difficult. Yet, our Landsat time-series demonstrated that the trends are persistent, with the strongest decrease in the period 2008-2011.

Given the large reed area that is persistently present, it is clear that the ecosystem services are clearly not threatened by reed die-back. However, a too strong reed expansion might still be a risk for service stability. Anthropogenic influences have increased influence of the Danube river and limited exchange with the Black Sea during the past century (Hanganu et al. 1999, 2002, Stanica et al. 2012). As a result the lagoon system has become mainly a fresh water system and is collecting organic and non-organic matter (Stanica et al. 2012), with the Sinoe Lagoon the only notable exception, as it has turned towards natural evolution and a trend 
towards brackish waters since mid 2000s, when the previously controlled engineered inlets were left in natural flow. Reed is known for its capability to absorb nutrients such as nitrogen from water (Findlay et al. 2002), because of this ability it is often applied in helophyte filters. This means it will clean water passing through the lagoon, but also trap many nutrients there.

If the system continues to accumulate sediments and the elevation increases to the point where it offers suitable growing conditions to reed throughout the lagoon, a sudden explosive reed expansion could threaten open water dependent ecosystem services and the corresponding economic benefits. To ensure long term service stability, this situation will have to be monitored carefully.

Our analyses showed that reed expansion mainly occurred in sheltered areas, whereas exposed areas were mainly stable or decreasing. An increased establishment at sheltered sites was also indicated by Coops et al. (1994) in the Netherlands. In contrast, Weisner (1987) found a rapid expansion of reed at exposed sites in Sweden. However, Weisner (1987) pointed out that an elevation difference likely created by a different grazing regime might be the underlying cause for the rapid expansion. In general, Weisner (1987) too expected a more rapid expansion at sheltered sites. This may be associated with wave forcing, which is known to have a large influence on reed development (Haslam 1970, Coops et al. 1991), and has been shown to effect seedling establishments in other marsh species (Cao et al. 2019).

\subsubsection{Conclusions and outlook}

We developed an indicator that can help predict the stability of reed wetlands, based on the size of seasonal differences in radar backscatter. We found that decreasing areas had smaller seasonal changes than stable or increasing areas. Radar showed differences among expanding, stable and retracting reed beds, where optical data did not. Although further study to pinpoint the biophysical processes underlying the radar signal would be recommended, its application has already proven to be very useful. We expect that this technique can easily be adapted to similar ecosystems such as salt marshes, but can also be applied to terrestrial systems such as heathlands or grasslands. In heathlands, SAR is also known to contribute new information not obtainable through optical data alone (Schmidt et al. 2018) and heathlands are known to adjust their seasonal development based on local conditions (Specht et al. 1983). This technique might even be applied to forests, where SAR is already often used to establish biomass 
(Luckman et al. 1998), but seasonal variation has not yet been used to assess the service stability. However, the great difficulties associated with mapping foliage dynamics in forests using SAR (Proisy et al. 2000), suggests that the radar backscatter indicator identified in the current study is likely best suited to characterize highly dynamic systems, which produce large seasonal differences. 
Chapter 4: Indicators of expansion and retreat of Phragmites based on optical and radar satellite remote sensing: a case study on the Danube delta

\section{Appendix 4.1, map of Romanian sample sites}

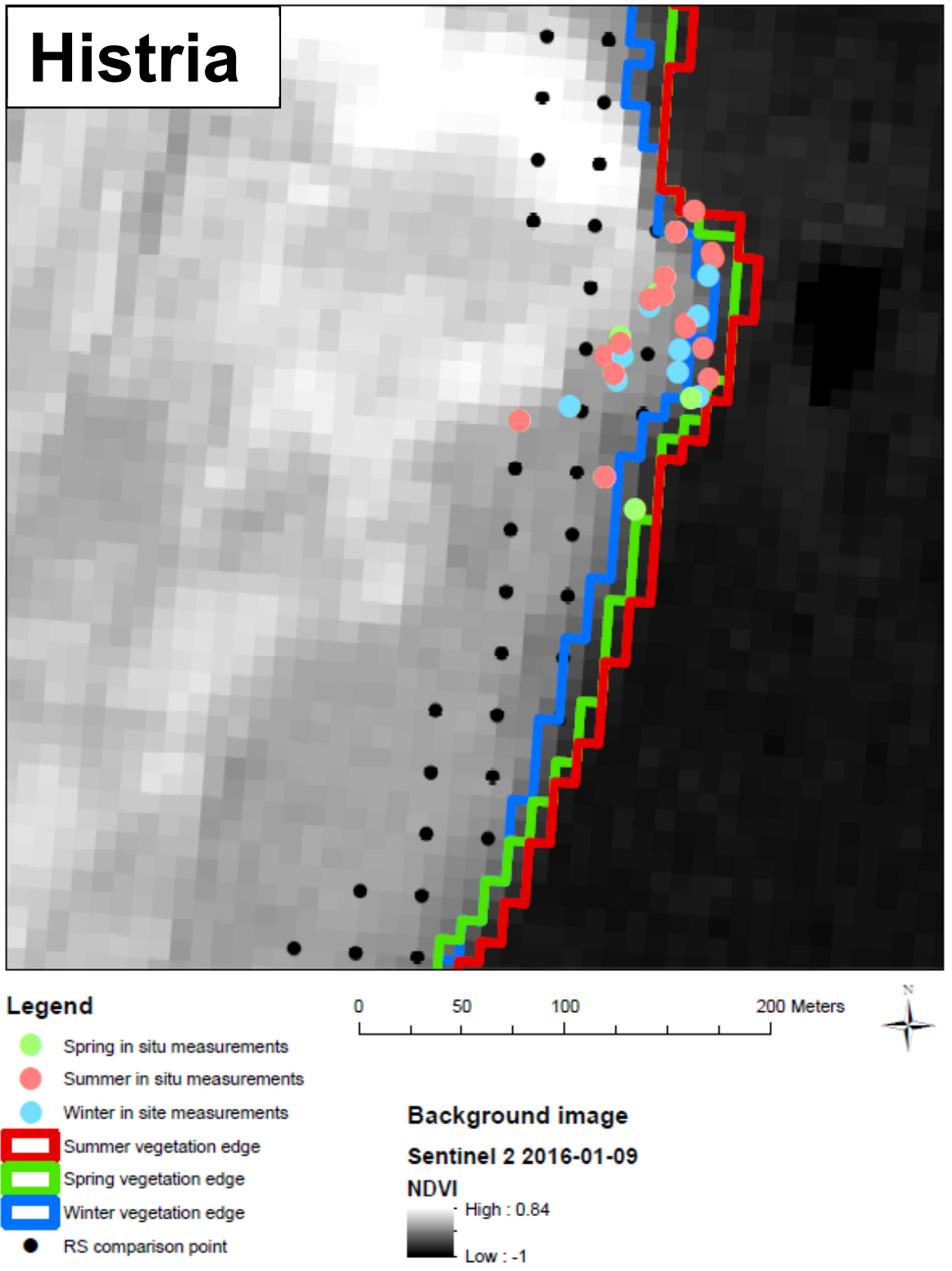


Chapter 4: Indicators of expansion and retreat of Phragmites based on optical and radar satellite remote sensing: a case study on the Danube delta

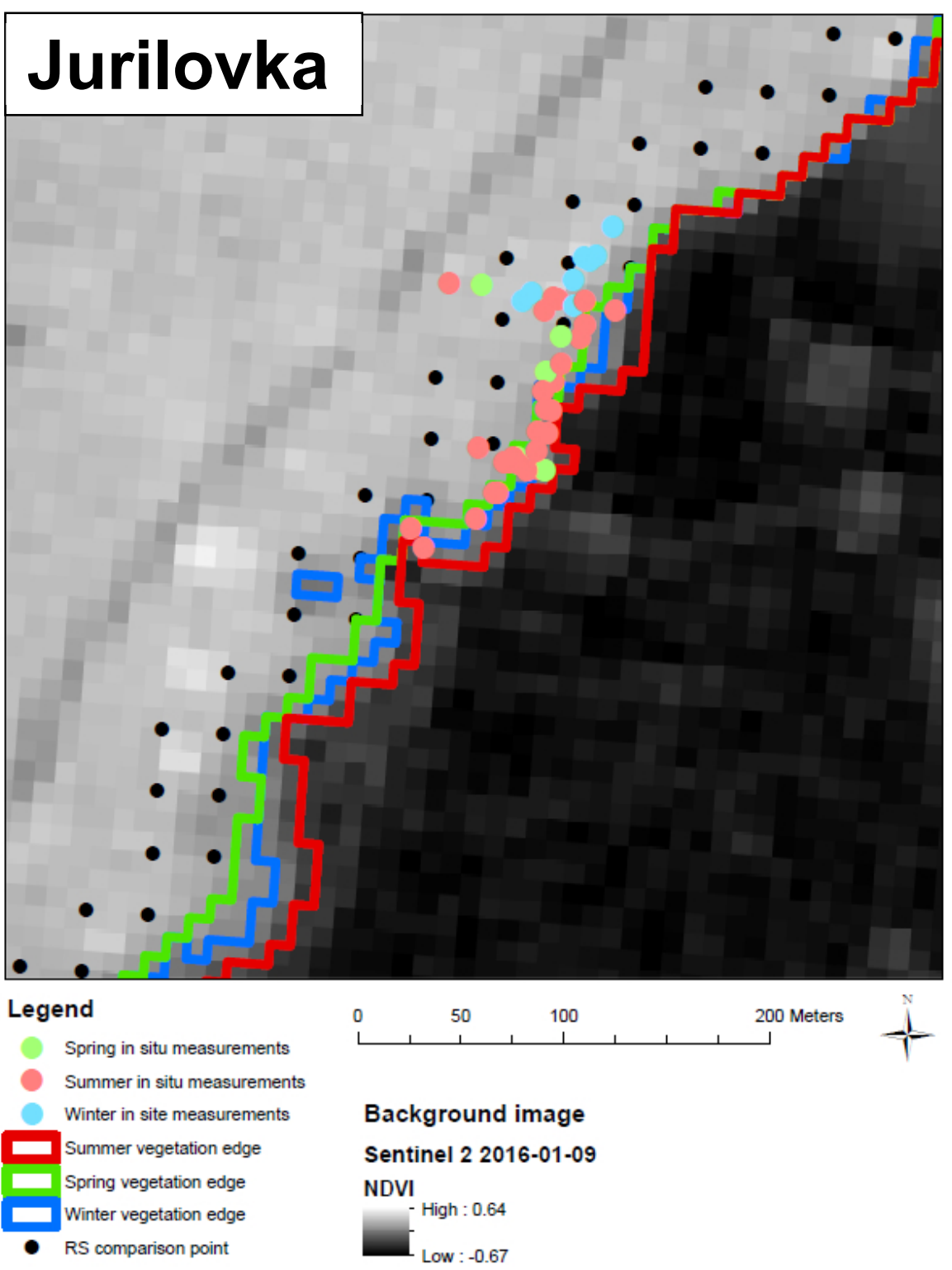


Chapter 4: Indicators of expansion and retreat of Phragmites based on optical and radar satellite remote sensing: a case study on the Danube delta

\section{Appendix 4.2, cloud masks}

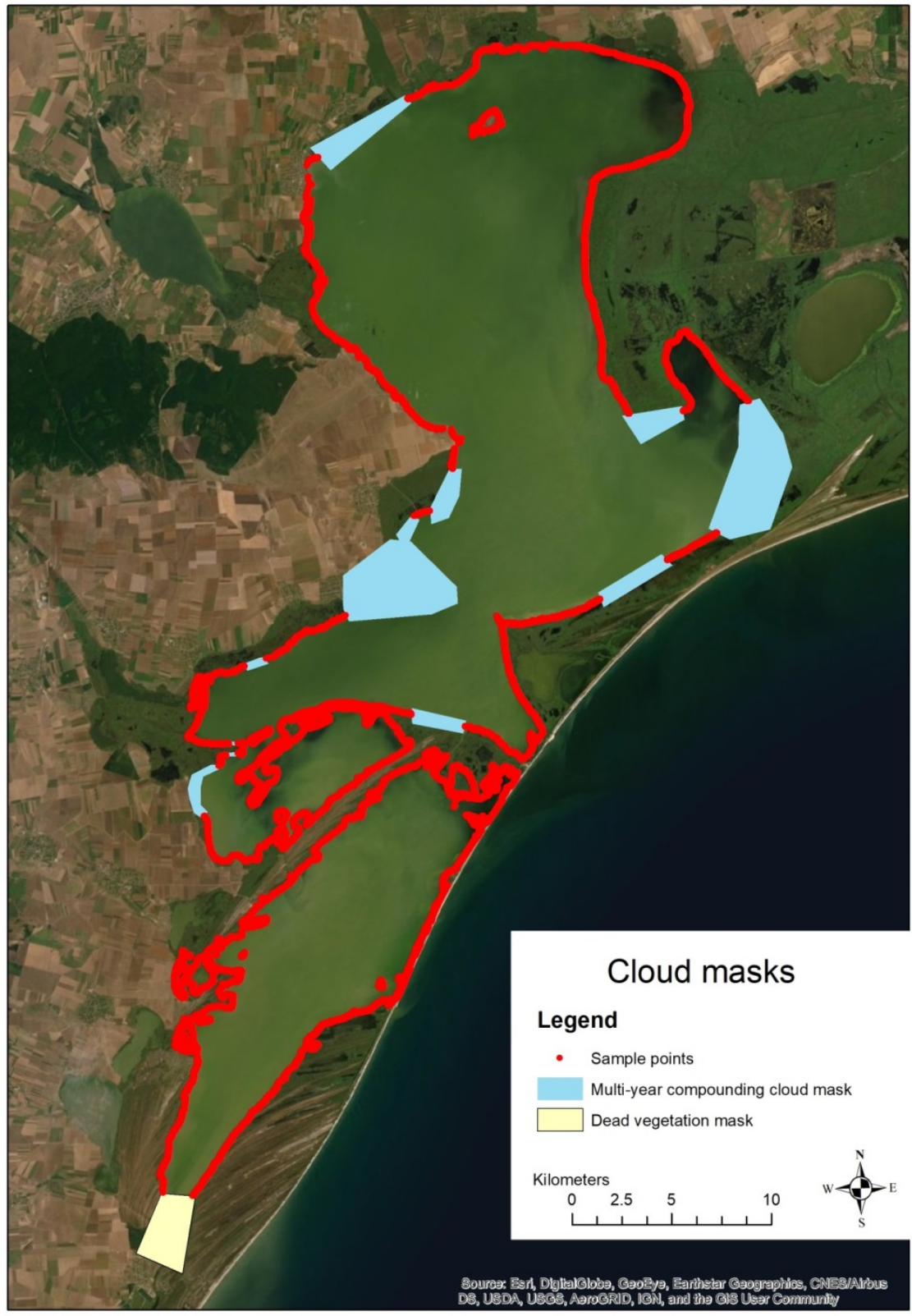




\title{
Chapter 5: Stress in salt marshes at leaf, plant and community level; towards predicting ecosystem development from satellite remote sensing
}

\section{Authors:}

B. Oteman ${ }^{1}$, T.J. Bouma1 ${ }^{1}$, D. van der Wal ${ }^{1,2}$

1 NIOZ Royal Netherlands Institute for Sea Research, Department of Estuarine and Delta Systems, and Utrecht University, P.O. Box 140, 4400 AC Yerseke, the Netherlands.

2 Faculty of Geo-Information Science and Earth Observation (ITC), University of Twente, P.O. Box 217, 7500 AE Enschede, The Netherlands.

*correspondence: bas.oteman@nioz.nl, TEL: +31 (0)113 577300

\begin{abstract}
Shifts in vegetation composition can change the services provided by an ecosystem. Hence, being able to monitor vegetation stress as a potential indicator of pending shifts in vegetation composition is important. Salt marshes are vegetated ecosystems providing a wide range of ecosystem services to humanity. Salt marshes are characterized by clear stress gradients along their elevational gradients, making them an ideal system to identify potential stress indicators. We compare stress indicators at leaf level (i.e., pigments, as well as leaf reflectance indices), plant level (i.e. cover, biomass and plant recovery rate after disturbance) and community level (i.e., vegetation height, vegetation structure, community reflectance indices and community recovery rate). We analyze how potential stress indicators change along an elevation gradient in two different saltmarsh sites in the Netherlands, dominated by the pioneer species Spartina and Scirpus, respectively. Here, we expect increased stress at lower elevations due to flooding and increased stress at higher elevations due to competition.

We found that, at the leaf-level, pigments such as chlorophyll-a and reflectance indices responded significantly to inundation stress. In contrast to expectations, values were highest at lowest elevations. At the plant-level, vegetation cover and biomass indicated stress well, and typically showed a clear optimum at intermediate elevations. Hence they are recommended as indicator for monitoring very sensitive areas, but will be difficult to apply on a large scale as they are labor intensive, and biomass sampling is typically destructive.

On both the plant and community level, the dependence of recovery rate on elevation differed strongly between species and between seasonal timing. Due to
\end{abstract}


this complex response, recovery rate after disturbance is unlikely applicable as a generic stress indicator in salt marshes.

At the community-level, reflectance correlated strongly with elevation, the strongest response was caused by inundation stress. The lowest reflectance values occurred at the lowest elevations. However the choice of vegetation index derived from reflectance seems very important. The often used Normalized Difference Vegetation Index was a clear function of inundation stress in one of our study species, but not in another, likely due to saturation. Overall, our analyses indicate that the choice of the most suitable reflectance based Vegetation Index strongly depends upon the studied species. Local system knowledge therefore remains vital for prediction of stress based on remote sensing.

Key words: Salt marsh, vegetation stress, resilience, structure analysis, pigment, vegetation reflectance

\subsection{Introduction}

Ecosystems provide numerous valuable services to humanity, with vegetated coastal systems being especially valuable (Costanza et al. 1998). Salt marshes are valuable, because they reduce nutrient loading of coastal waters (Chmura 2011) and provide flood protection through wave attenuation (Möller 2006, Barbier et al. 2008, Temmerman et al. 2013, Zhu et al. 2020). Moreover, they provide economic benefits by increasing tourism (Henderson and Lewis 2008, Chmura 2011) and by sustaining fish (Boesch and Turner 1984, Deegan et al. 2002, Chmura 2011), plants and other animals valued for human consumption (Henderson and Lewis 2008, de Groot et al. 2011). Because these services are related to the presence of specific plant species or communities consisting of specific plant species, the stability of these services can often only be ensured by ensuring the stability of the entire system. Monitoring the stability of vegetation at the ecosystem level is often done by examining species composition over time. Given the ongoing climate change including potential sea level rise and increase in storminess (Pethick 2001, Vilibić and Šepić 2010), which both affect coastal marshes, there is a need for indicators that can be monitored to predict if species composition will shift. Hence, in this study we aim to provide a first step towards monitoring stress in salt marsh vegetation, as an indicator of its stability.

In this study we define stress as anything that reduces vegetation growth, similar to Lichtenthaler's definition of dis-stress (Lichtenthaler et al. 1996). In this study we focus solely on such dis-stress. Natural changes in vegetation type are typically accompanied by stress, causing the habitat to become less suitable for the species 
that is currently there compared to the species that is taking over. This makes methods that allow quantifying vegetation stress promising as potential indicator of pending vegetation changes, which in turn gives an indication of system stability. A disturbance is here defined as a sudden and often lethal event such as the removal of all above ground biomass.

Any environmental factor can potentially be a stressor, hence a wide variety of specific types of stress can occur (e.g. saline stress, nutrient toxicity or herbivory). Most stressors directly or indirectly affect the photosynthetic process (Lichtenthaler 1996), so that stress can be detected at the leaf level by measuring the chlorophyll content (Carter and Knapp 2001b, Zarco-Tejada et al. 2002). Alternatively, at the plant level, the biomass can be sampled as indicator of stress, as a reduction of photosynthesis decreases the biomass development potential. Both in nature conservation and agriculture vegetation, stress is often analyzed using biomass. Destructive sampling for biomass determination is typically feasible for small representative plots, but is less practical for establishing stress on a large scale for monitoring purposes.

Vegetation stress can also be analyzed at community level, by establishing cover for each species. This is called phytosociology and started more than forty years ago (Van der Maarel 1971). In phytosociology plant cover of different species is combined into a specific community type. These community types can be linked to indicator values to assess an individual type of stressor (Ellenberg et al. 1991). Vegetation height is often measured in addition to vegetation cover, to get a better approximation of biomass (Introduced by: Barkman 1979). Additionally, vegetation structure might be a useful stress indicator at the community level (Oteman et al. publication pending). Both agriculture and nature conservation are increasingly using remote sensing systems to establish stress at the community level. These studies mostly depend on physiological attributes that affect light reflectance. Reflectance indices such as the Normalized Difference Vegetation Index (NDVI) are widely applied in vegetation studies (Pettorelli et al. 2005).

In salt marshes, two main stressors are flood duration (the length of time plants are inundated) and competition. Both are known to have a strong influence on both plant health and vegetation zonation (Bertness and Ellison 1987, Silvestri et al. 2005). In this study we focus on the effects of these two main stressors along an elevation gradient. Recently a study found flood duration, and associated intertidal elevation, to be the most important driver behind spatial patterning in a Dutch salt marsh (Oteman et al. 2019). The effect of flood duration was found to be four times as large as any other cause behind spatial patterns, such as creek formation and 
wave forcing (Oteman et al. 2019). This shows that elevation in intertidal systems, can be expected to be a proxy for inundation stress. Transplantation experiments have shown that elevation also plays an important role in competition (Bertness and Ellison 1987); it co-determines vegetation zonation (Bertness and Ellison 1987, Silvestri et al. 2005) and it affects the recovery rate in salt marshes, where vegetation at the lower edge requires a longer time to recover after a disturbance (van Belzen et al. 2017).

The alternative stable states theory (Scheffer et al. 2001, 2009) suggests that vegetation stress indicators might not be linearly related to the stressor, and plants can appear unstressed until a tipping point is reached after which the vegetation can suddenly collapse or change to another vegetation type. The underlying cause behind these sudden collapses is always a feedback that stabilizes the system. Once such a feedback stops, the system rapidly deteriorates. Because the system is kept stable by a feedback mechanism, these systems are unlikely to be represented well by normal stress indicators (Scheffer et al. 2009, Kéfi et al. 2013). In feedback dominated systems it is recommended to use recovery rate as an indicator for stability; closer to a tipping point a system will take longer to recover from a perturbation. This phenomenon is called critical slowing down. A recent study found proof that salt marshes have a significantly lower recovery rate at lower elevation (van Belzen et al. 2017), indicating that critical slowing down also occurs in salt marshes, and responds to elevation. Critical slowing down has also been suggested as a more general stress indicator, to monitor systems and detect pending change (Kéfi et al. 2013). In this paper, we test whether recovery rate after disturbance is a suitable indicator in a stress-based monitoring system.

To support salt marsh monitoring and to help ensure the ecosystem services they provide remain stable, we analyze how sensitive different stress indicators are to elevation, as a reliable salt marsh stress indicator should respond strongly to elevation. We study indicators (i.e., chlorophyll content, vegetation cover, vegetation height, leaf reflectance, community reflectance, structure and recovery rate) at three levels: leaf, plant and community level. At leaf level, we look at pigments and reflectance; at plant level, we study cover, biomass and plant recovery rate; at the community level, we look at vegetation height, community reflectance, vegetation structure and community recovery rate (See Table 1). We hypothesize that sensitive stress indicators indicate a high stress level at low elevations, where they respond to prolonged inundation. At intermediate elevation we expect optimum conditions (i.e., lowest stress levels). At high elevation we expect increased stress due to competition. For indicators that are positively related with the two stressors, a linear increase in the value of these stress 
indicators with elevation would therefore suggest sensitivity to inundation stress at the seaward edge of the vegetation only, while a decrease in these stress indicators would point to sensitivity to competition stress only. We evaluate these stress indicators along elevation gradients in two different saltmarsh sites in the Netherlands, dominated by the pioneering species Spartina and Scirpus, respectively.

\subsection{Method}

\subsubsection{Study sites}

We sampled two Dutch salt marshes 'Paulina' and 'Rilland', both along the Westerschelde estuary. The Westerschelde is a macrotidal estuary, experiencing semi-diurnal tides, with a mean tidal range of $4.18 \mathrm{~m}$ (station Terneuzen) near Paulina and 4.94m (station Bath) near Rilland (Rijkswaterstaat, 2019). High water at spring tides reaches $2.29 \mathrm{~m}$ NAP at Paulina and $2.75 \mathrm{~m}$ NAP at Rilland, respectively. Both sites are located in the polyhaline zone of the estuary, with salinities in Paulina, closer to the estuarine mouth, higher than in Rilland. Paulina is located at $51.35^{\circ}$ latitude and $3.72^{\circ}$ longitude. The saltmarsh consists mainly of the pioneer species common cord grass (Spartina anglica), a standing grass that can cope with regular flooding. In addition, sea couch grass (Elytrigia atherica), a lying grass, and sea purslane (Atrixplex portulocoides), a small shrub species, are abundantly present. Spartina is a stress resistant species, which occurs nearest to the water, but is outcompeted at higher elevations.

Rilland, located at $51.40^{\circ}$ latitude and $4.17^{\circ}$ longitude, is a salt marsh dominated by Scirpus maritimus and Phragmites australis. Higher in the marsh other grass species such as Agrostis stolonifera, Elytrigia atherica and Festuca rubra also occur. Scirpus too is a pioneering species, which also occupied the lowest elevations. Scirpus does not occur in the Paulina fieldsite, Spartina only very rarely occurs at Rilland and was not found along any transect or in any of the studied plots at this site. Both species are the primary pioneering species in their respective field sites, and cover large enough areas to ensure their local populations are not threatened by our experimental disturbance. 


\subsubsection{Experimental and measurement design}

To gain insight into the response of potential stress indicators to elevation, we placed three transects along the elevation gradient in each of the two study areas, yielding six transects in total. Each transect started at the vegetation closest to the water and ran perpendicular to the water line landward, until the target species (Spartina in Paulina and Scirpus in Rilland) no longer occurred. The next ten meters were checked to ensure the species no longer occurred. Along each transect, two $1 \times 1$ meter plots were placed every 5 meters. A primary plot was placed directly in line with the transect, a second plot which was manually checked to have the same vegetation composition was placed as closely as possible. The coordinates of the center point of both plots were measured with a differential GPS to establish elevation, and the corners were marked with bamboo sticks. At each plot we measured chlorophyll content and reflectance at leaf level, vegetation biomass and cover at plant level and vegetation height, vegetation cover, vegetation structure, biomass, reflectance and recovery at the community level (see table 1). One of the plots was used as control while the other was experimental. In the experimental plots all above ground biomass was removed in late spring/early summer (Spartina: June-July, Scirpus July-Early August). Recovery was recorded in May the next year. 
Table 5.1. Overview of measurement levels.

\begin{tabular}{llll}
\hline Measurement & Leaf level & Plant level & Community level \\
\hline Pigments & $\mathrm{x}$ & & \\
Reflectance & $\mathrm{x}$ & $\mathrm{x}$ & \\
Cover & & $\mathrm{x}$ & \\
Biomass & & $\mathrm{x}$ \\
Height & & $\mathrm{x}$ \\
Structure & $\mathrm{x}$ & $\mathrm{x}$ \\
Recovery - cover & & $\mathrm{x}$ \\
Recovery - height & & & \\
\hline
\end{tabular}

\subsubsection{Leaf level measurements}

Reflectance was measured at leaf and at community level, using a TriOS Ramses 842D spectroradiometer. This spectrometer has a spectral range of $320-950 \mathrm{~nm}$, with a spectral resolution of $3.3 \mathrm{~nm}$. At each plot a random selection of $10-30$ mature top leaves were collected and arranged into a surface of approximately 8 by $10 \mathrm{~cm}$. This surface was placed at $16 \mathrm{~cm}$ from the spectroradiometer (to measure a circle of with a diameter of $1 \mathrm{~cm}$ ). Each surface was sampled five times, measurements did not overlap. Between each measurement, the spectrum of a white Styrofoam panel was measured as a reference for down welling irradiance. Surface reflectance were calculated from the spectra, following (Kromkamp et al. 2006). From the surface reflectance we calculated two often used indices: the Normalized Difference Vegetation Index (NDVI) and the Red Edge Inflection Point (REIP). The NDVI was calculated using: $\left(R_{750}-R_{675}\right) /\left(R_{750}+R_{675}\right)$. The REIP was calculated using: $700+40\left(\left(R E-R_{700}\right) /\left(R_{740}-R_{700}\right)\right)$ where $R E=\left(R_{670}+R_{780}\right) / 2$; following (Clevers et al. 2002). In these formulas $R_{670}, R_{675}, R_{700}, R_{740}, R_{750}$ and $R_{780}$ are surface reflectances at wavelengths of $670,675,700,740,750$ and 780 $\mathrm{nm}$ respectively.

After the leaf surface reflectance measurements, the leaves were immediately frozen and brought to the laboratory for pigment analysis. High-performance liquid chromatography (HPLC) was used to extract chlorophyll A (Chl-a), B (Chl-b), and Carotene (car). For a more detailed description of reflectance measurements and pigment collection see (Oteman et al. 2019). 


\subsubsection{Plant level measurements}

At plant level, cover and biomass were measured, and cover development after disturbance was assessed. The cover was recorded for both the disturbed and control plots. The cover was estimated manually by an expert to a precision of $1 \%$. If the cover was below $1 \%$, it was rounded to either 0.5 or $1 \%$. Species with a cover lower than $0.25 \%$ were not taken into account. To establish relative recovery, the cover at the disturbed plots was taken as a fraction of the cover at the control plots.

Biomass was harvested in a 20x20 cm area and separated between species. For each species the biomass was weighed, dried for 5 days in an oven at 65 degrees Celsius and weighed again, to establish both fresh and dry weight. Biomass was measured next to the control plots; these biomass plots were manually selected such that cover and height were similar to the control plots.

\subsubsection{Community level measurements}

At the community level we measured height, reflectance, structure and recovery rate. The community total cover and total biomass was considered, as the sums of the measurements of the different species at plant level. The vegetation height was recorded with the 'drop disk' method similar to (Van der Graaf et al. 2002): a polystryrene disk (24gram, $20 \mathrm{~cm}$ diameter) is used to estimate vegetation height as this takes plant strength into account and helps to avoid outliers of single long leaves (Barkman 1979). We recorded both the height that the disk first touches the vegetation (height top) and the height at which it rests on the vegetation (height disk). In each plot we used the average of 5 measurements, in a quincunx (dice five) pattern to avoid pseudo-replication. Vegetation height measurements are considered a community level measurement because when using a drop disk it is not possible to differentiate between species. Both height and cover were measured in the control and in the disturbed plot, the recovery rate was calculated as the cover or height in the disturbed plot as a fraction of the cover or height in the control plot.

Reflectance at community level was measured using a specially designed rig to hold the spectroradiometer stable at 2 meters height, this gives a measurement radius on the ground of $20 \mathrm{~cm}$. As with the measurements at leaf level, a white Styrofoam panel was used as a reference before each measurement (following: Kromkamp et al. 2006). This was done 5 times in each plot (for more details see: Oteman et al. 2019). 
Finally, vegetation structure was measured using the technique 'depth from focus' described by (Oteman et. al, publication pending). This technique allows for the creation of a $3 d$ representation of vegetation using a DSLR camera. We calculated the vertical surface roughness RMSz (standard deviation of all heights $\mathrm{z}$ ) and the horizontal structure parameter Moran's-I (denoting spatial autocorrelation) using the raster package in r. For Moran's-I we used the queen pattern and a window size of $201 \times 201$ pixels, which roughly translates to $4 \times 4 \mathrm{~cm}$.

\subsubsection{Data analysis}

We expect two types of main stressors along the elevation gradient, hydrodynamic stress, which is strongest at low elevations, and competition related stress, which is strongest at high elevation. A stress indicator can correspond to one of these and give a linear response to the stressor, or to both which produces an initial increase followed by a decrease or vice versa. To test these effects, we fit a standard second degree linear model and a quadratic model through the indicator as a function of elevation. This is done for each variable. We use the coefficient of determination $R^{2}$ (and significance level $p$ ) to establish how sensitive the potential indicators are to elevation. The Akaike Information Criterion (AIC) is used to determine if the linear or quadratic model performs best (i.e., has the lowest AIC). All statistical analyses were performed in $r$.

It is important to note that we removed all plots that contained multiple species from the community indicators, as these indicators cannot distinguish between species. 


\subsection{Results}

\subsubsection{Leaf level}

At the leaf level, we examined pigments and reflectance. The pigment analysis showed a linear decline of the pigments with elevation in Spartina vegetation, pointing to stress due to competition (see table 5.2 and figure 5.1). Scirpus shows that chlorophyll content is best represented by a concave-down quadratic curve, i.e., an optimum in chlorophyll at intermediate elevations, indicating lowest stress levels (see table 5.3 and figure 5.2). The lower $R^{2}$ values (explaining $17 \%$ of the variation or less) indicate the relation is not as strong as with Spartina.

The leaf reflectance of Spartina shows that the relation between both the NDVI and REIP and elevation are best represented by a quadratic model, with an initial increase followed by a decrease at higher elevations (see table 5.2 and figure 5.2), pointing to stress both at low and high elevations. NDVI as a function of elevation has a relatively high $R^{2}$ of 0.31 , the REIP as a function of elevation has a $R^{2}$ of 0.11 . Thus, NDVI relates well with elevation, whereas REIP does not. Scirpus shows a poor relationship between REIP and elevation, and a significant but noisy relationship between elevation and NDVI (see table 5.3 and figure 5.2); in this case NDVI has the lowest values at intermediate elevations.

\subsubsection{Plant level}

At the plant level, cover and biomass of both Spartina and Scirpus show a similar pattern, a parabolic curve with a clear optimum at intermediate elevation (see table $5.2+5.3$ and figure 5.3). Both relationships have relatively high $R^{2}$ values. The plant level cover recovery showed an increased recovery rate further from the sea in both species, but this was much more pronounced in Scirpus than in Spartina.

\subsubsection{Community level}

At the community level, we only analyze mono-specific plots, to avoid the variation introduced by other species. Spartina does not show a significant relation between vegetation height and elevation (see table 5.2 and figure 5.4). Scirpus shows a linear increase in vegetation height as well (see table 5.3 and figure 5.4). The prevalence of linear models here is likely due to the limitation of the effects of competition, as multi-species plots are excluded. 
The NDVI showed a similarly linear pattern with lowest values at low elevations, pointing to stress due to inundation, although in Scirpus the relationship between NDVI and elevation was not significant, possibly because the NDVI values had saturated at higher elevations. The REIP related strongly with elevation in Spartina, and reasonably strongly in Scirpus (see table 5.2 and 5.3 and figure 5.5), again with lowest values at lowest elevation.

The structure indicators are mostly non-significantly related to elevation, with the exception of Moran's-I in Spartina, which has a quadratic relation with elevation (which explains $20 \%$ of the total variation). Moran's I had the lowest values at intermediate elevations. The height recovery of Spartina and Scirpus is not significantly related to elevation. The recovery rate of Spartina also shows no significant relationship with elevation, while the recovery rate of Scirpus has a clear linear relation with elevation, with lowest recovery rate at lowest elevations where inundation stress is high. 


\subsubsection{Biophysical properties}

Table 5.2. Relation of leaf, plant and community variables with elevation for Spartina. Chl-a and Chl-b stand for chlorophyll a and b respectively. Car stands for Carotene. NDVI means Normalized Difference Vegetation Index and REIP stands for Red Edge Inflection Point.

\begin{tabular}{|c|c|c|c|c|c|c|c|c|c|}
\hline & & & & Linear & & & Quadratic & & \\
\hline Species & Level & Var & $\begin{array}{l}\text { Best } \\
\text { model }\end{array}$ & & $\mathrm{R} 2$ & A.IC & & $\mathrm{R} 2$ & A.IC \\
\hline Spa & Leaf & Chl-a & Linear & $<0.01$ & 0.50 & 619.87 & $<0.01$ & 0.50 & 621.13 \\
\hline Spa & Leaf & chl-b & Linear & $<0.01$ & 0.37 & 521.38 & $<0.01$ & 0.37 & 522.55 \\
\hline Spa & Leaf & $\mathrm{car}$ & Linear & $<0.01$ & 0.47 & 423.32 & $<0.01$ & 0.46 & 425.18 \\
\hline Spa & Leaf & NDV $\mid$ & Quadratic & 0.02 & 0.11 & -92.92 & $<0.01$ & 0.32 & -102.73 \\
\hline $\mathrm{Spa}$ & Leaf & REIP & Quadratic & 0.85 & -0.03 & 93.54 & 0.05 & 0.11 & 88.97 \\
\hline Spa & Plant & Cover & Quadratic & 0.12 & 0.04 & 372.95 & $<0.01$ & 0.31 & 360.49 \\
\hline Spa & Plant & Biomass & Quadratic & 0.02 & 0.10 & -194.16 & $=0.01$ & 0.43 & -211.10 \\
\hline Spa & Plant & $\begin{array}{l}\text { Cower } \\
\text { recovery }\end{array}$ & Quadratic & 0.04 & 0.09 & 88.69 & 0.02 & 0.14 & 87.05 \\
\hline Spa & Community & Height disk & $\mathrm{ns}$ & 0.09 & 0.10 & 112.17 & 0.26 & 0.04 & 114.17 \\
\hline Spa & Community & Height top & ns & 0.29 & 0.01 & 136.36 & 0.56 & -0.04 & 138.29 \\
\hline Spa & Community & MDVI & Linear & 0.00 & 0.31 & -32.28 & 0.01 & 0.31 & -31.28 \\
\hline Spa & Community & REIP & Quadratic & 0.00 & 0.40 & 84.97 & 0.00 & 0.51 & 81.59 \\
\hline Spa & Community & Moran-I & Quadratic & 0.05 & 0.15 & -154.43 & 0.05 & 0.20 & -154.91 \\
\hline Spa & Community & $\begin{array}{l}\text { RMSL } \\
\text { Cover }\end{array}$ & ns & 0.97 & -0.05 & 102.94 & 0.82 & -0.09 & 104.47 \\
\hline Spa & Community & recovery & ns & 0.51 & -0.03 & 27.53 & 0.53 & -0.04 & 28.57 \\
\hline Spa & Community & $\begin{array}{l}\text { Height } \\
\text { recovery }\end{array}$ & $n s$ & 0.99 & -0.05 & 9.81 & 0.45 & -0.02 & 9.96 \\
\hline
\end{tabular}


Table 5.3. Relation of leaf, plant and community variables with elevation for Scirpus. Chl-a and Chl-b stand for chlorophyll a and b respectively. Car stands for Carotene. NDVI means Normalized Difference Vegetation Index and REIP stands for Red Edge Inflection Point.

\begin{tabular}{|c|c|c|c|c|c|c|c|c|c|}
\hline \multirow[b]{2}{*}{ Species } & \multirow[b]{2}{*}{ Level } & \multirow[b]{2}{*}{ Var } & \multirow[b]{2}{*}{$\begin{array}{l}\text { Best } \\
\text { model }\end{array}$} & \multirow{2}{*}{$\begin{array}{l}\text { Linear } \\
p\end{array}$} & \multirow[b]{2}{*}{$\mathrm{R} 2$} & \multirow[b]{2}{*}{$\mathrm{AIC}$} & \multirow{2}{*}{$\begin{array}{l}\text { Quadratic } \\
\mathrm{p}\end{array}$} & \multirow[b]{2}{*}{$\mathrm{R} 2$} & \multirow[b]{2}{*}{ AIC } \\
\hline & & & & & & & & & \\
\hline Sci & Leaf & Chl-a & Quadratic & 0.06 & 0.06 & 769.97 & 0.03 & 0.10 & 768.62 \\
\hline Sci & Leaf & chl-b & Quadratic & 0.15 & 0.02 & 645.81 & 0.05 & 0.09 & 643.54 \\
\hline Sci & Leaf & car & Quadratic & 0.01 & 0.13 & 521.49 & 0.01 & 0.17 & 520.08 \\
\hline $\mathrm{Sci}$ & Leaf & NDVI & Quadratic & 0.03 & 0.07 & -91.94 & 0.01 & 0.17 & -96.22 \\
\hline $\mathrm{Sci}$ & Leaf & REIP & ns & 0.12 & 0.03 & 129.58 & 0.14 & 0.04 & 129.90 \\
\hline Sci & Plant & Cover & Quadratic & 0.74 & -0.02 & 329.22 & $<0.01$ & 0.24 & 315.95 \\
\hline $\mathrm{Sci}$ & Plant & Biomass & Quadratic & 0.70 & -0.02 & -109.32 & $<0.01$ & 0.25 & -123.01 \\
\hline Sci & Plant & $\begin{array}{l}\text { Cover } \\
\text { recovery }\end{array}$ & Quadratic & $=0.01$ & 0.36 & 122.33 & $<0.01$ & 0.50 & 111.67 \\
\hline Sci & Community & Height disk & Quadratic & 0.00 & 0.54 & 18837 & 0.00 & 0.62 & 184.11 \\
\hline Sci & Community & Height top & Quadratic & 0.00 & 0.71 & 181.31 & 0.00 & 0.78 & 174.60 \\
\hline $\mathrm{Sci}$ & Community & NDVI & $\mathrm{ns}$ & 0.07 & 0.10 & -11.83 & 0.21 & 0.06 & -9.87 \\
\hline Sci & Community & REIP & Linear & 0.01 & 0.21 & 118.50 & 0.04 & 0.19 & 120.17 \\
\hline Sci & Community & Moran-I & ns & 0.20 & 0.02 & -388.66 & 0.33 & 0.01 & -387.25 \\
\hline Sci & Community & $\begin{array}{l}\text { RMSZ } \\
\text { Cower }\end{array}$ & ns & 0.86 & -0.02 & 401.81 & 0.53 & -0.02 & 402.47 \\
\hline $\mathrm{Sci}$ & Community & recovery & ns & 0.21 & 0.03 & 25.06 & 0.39 & 0.00 & 26.65 \\
\hline $\mathrm{Sci}$ & Community & $\begin{array}{l}\text { Height } \\
\text { recovery }\end{array}$ & Linear & 0.01 & 0.23 & 8.07 & 0.03 & 0.22 & 9.36 \\
\hline
\end{tabular}




\section{Leaf level stress indicators - pigments}
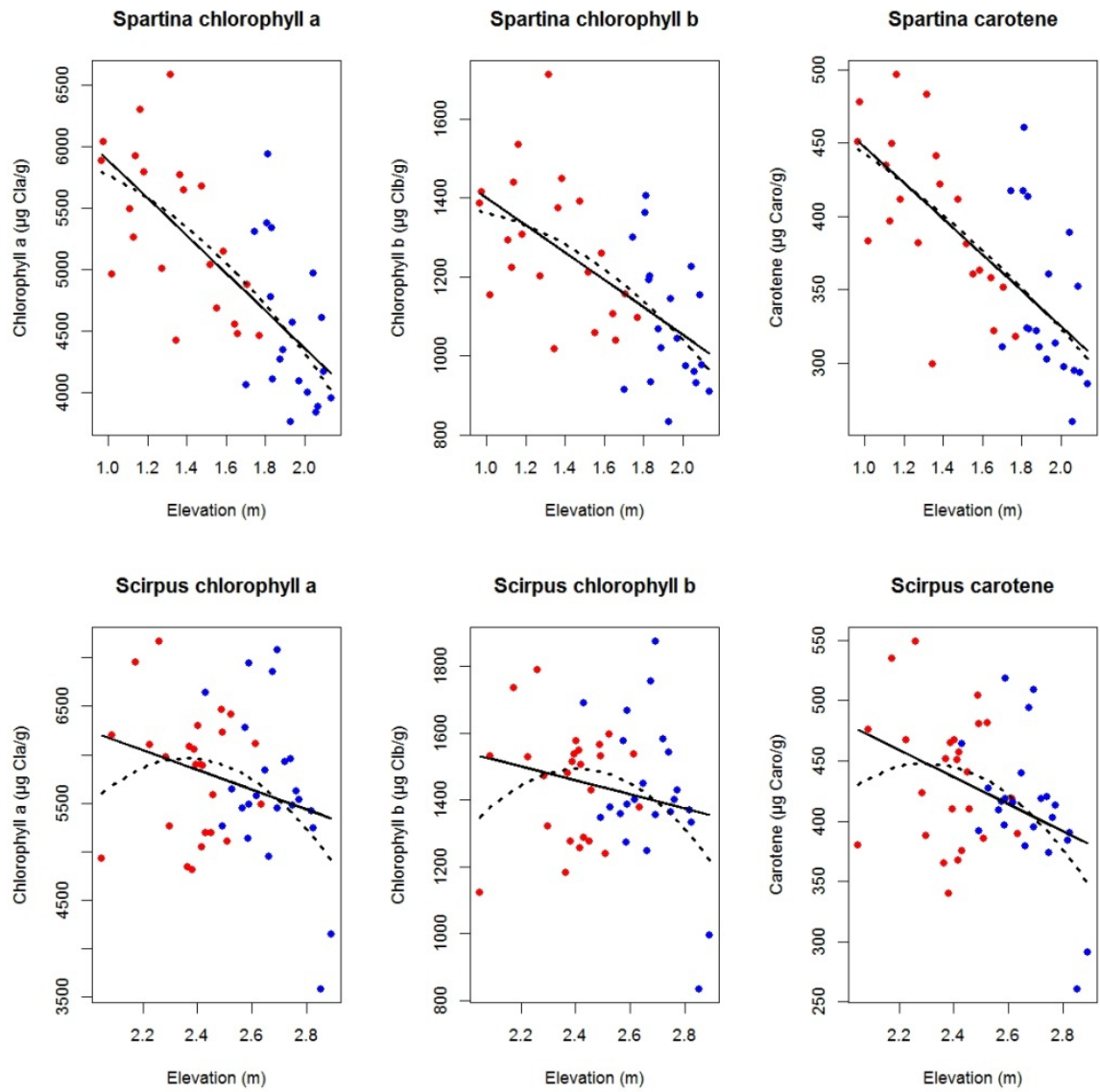

Figure 5.1, Relations of leaf level pigments with elevation. Blue points represent plots where other species (besides Spartina or Scirpus) also occurred, red points are plots that only have Spartina or Scirpus. The solid line is the linear model, the dotted line represents the quadratic model. 


\section{Leaf level stress indicators - reflectance}
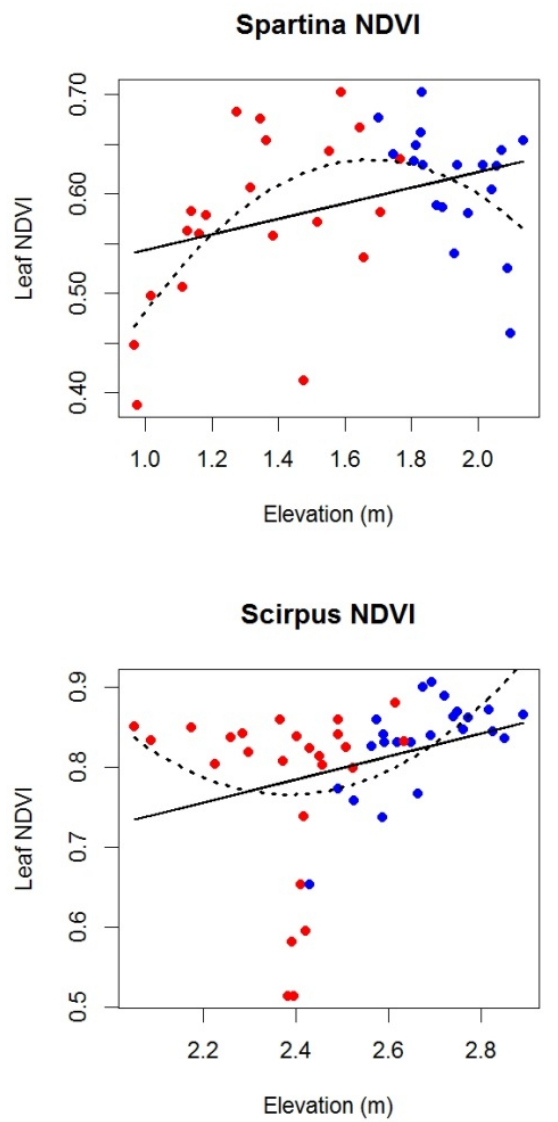

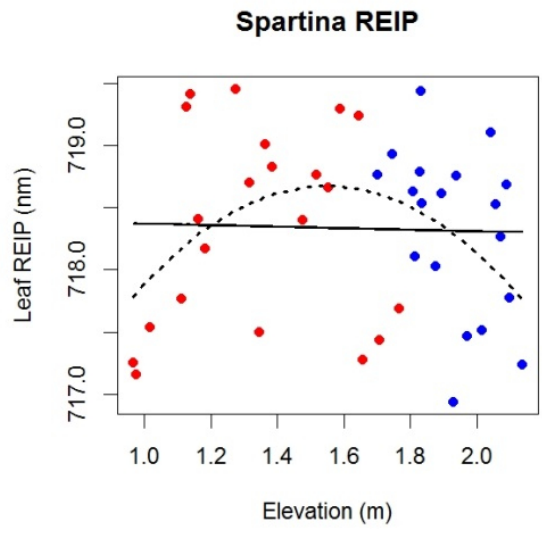

Scirpus REIP

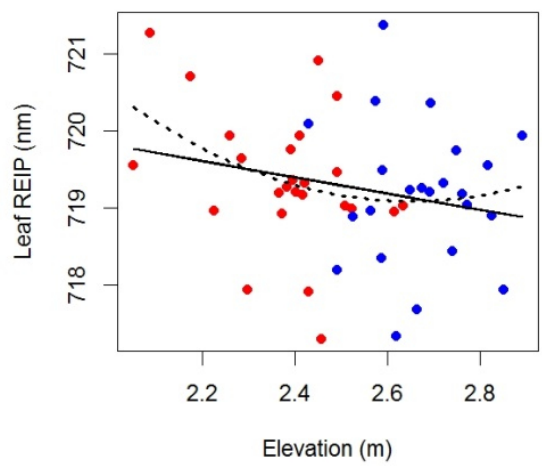

Figure 5.2. Relations of leaf level vegetation indices with elevation. Blue points represent plots where other species (besides Spartina or Scirpus) also occurred, red points are plots that only have Spartina or Scirpus. The solid line is the linear model, the dotted line represents the quadratic model. 


\section{Plant level stress indicators}
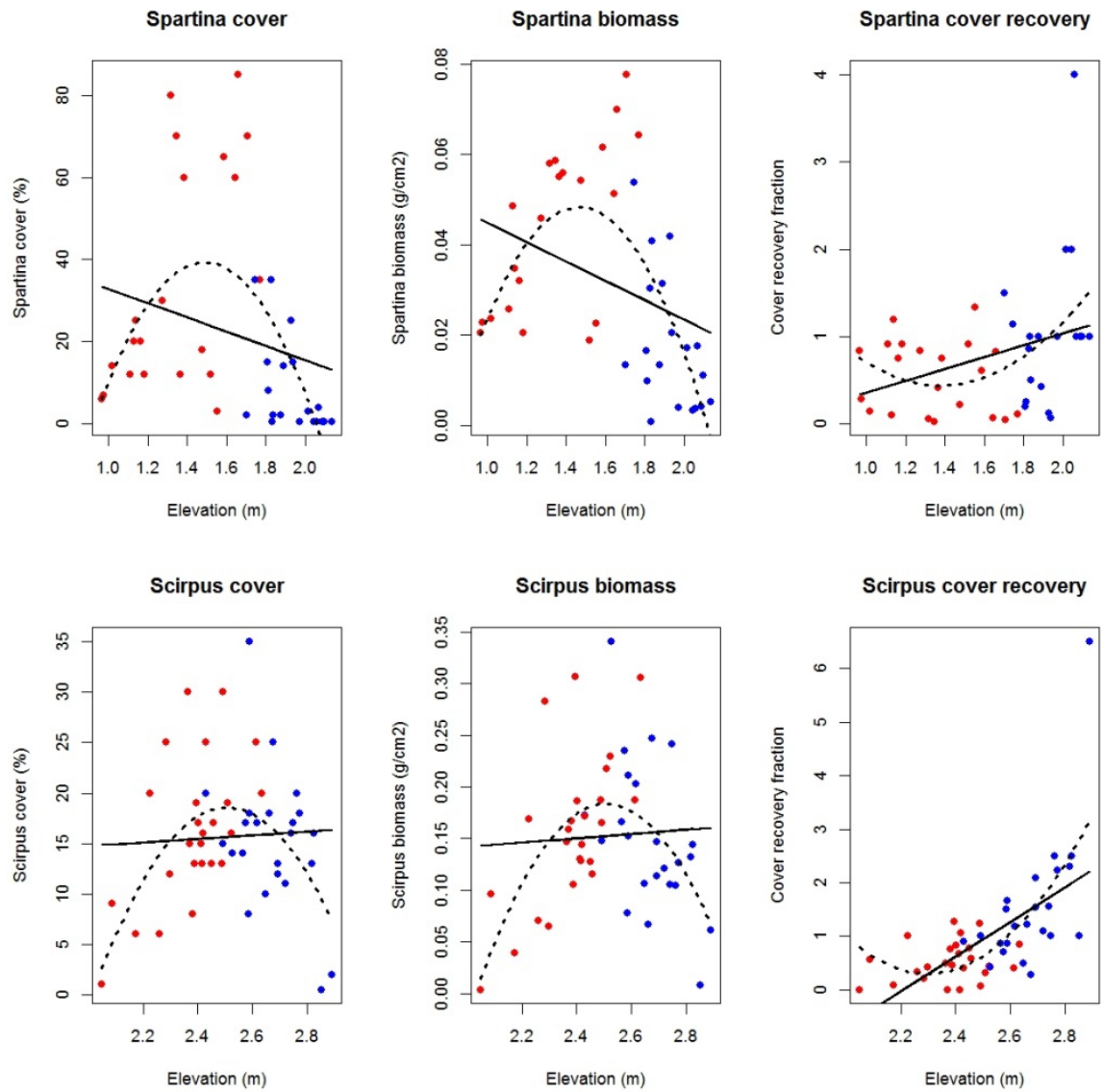

Figure 5.3. Relation of plant level stress indicators with elevation. Blue points represent plots where other species (besides Spartina or Scirpus) also occurred, red points are plots that only have Spartina or Scirpus. The solid line is the linear model, the dotted line represents the quadratic model. Recovery fractions is the fraction recovered relative to the corresponding control after 11 months. 


\section{Community level stress indicators - biophysical properties}

Spartina height

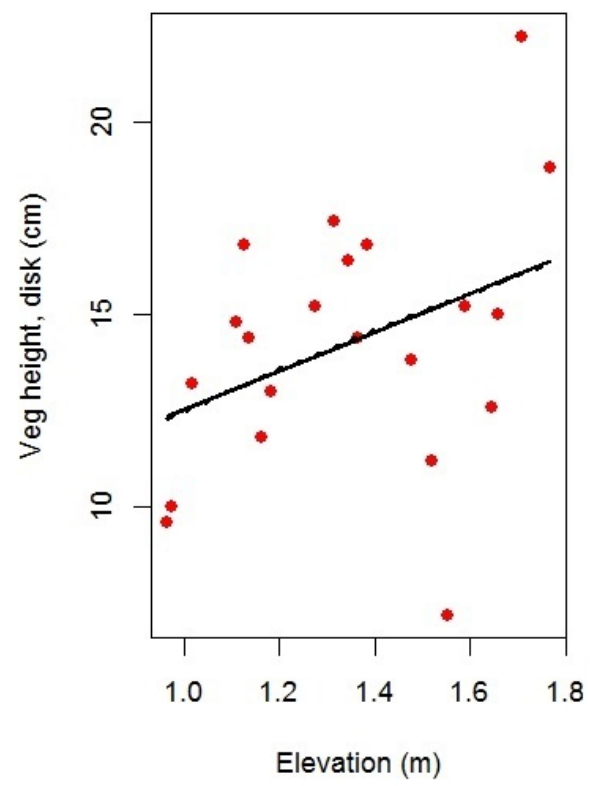

Scirpus height

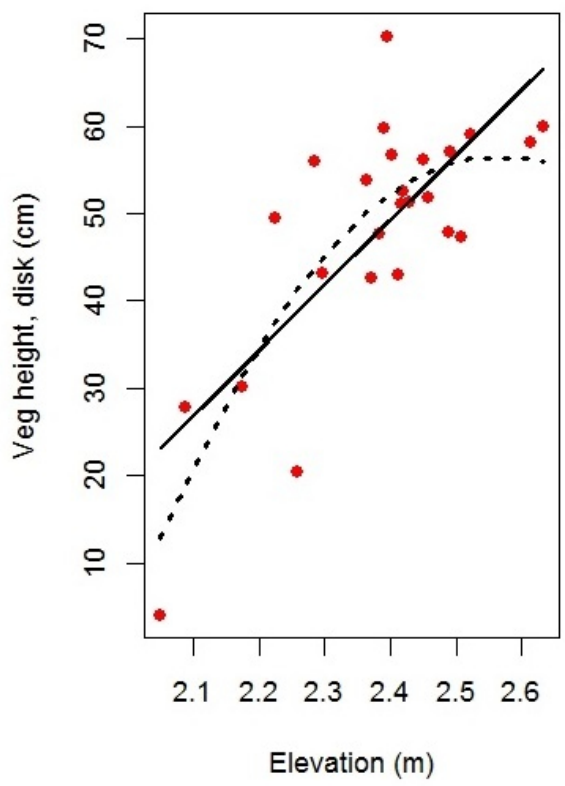

Figure 5.4. Relation of community level stress indicators with elevation. The solid line is the linear model, the dotted line represents the quadratic model. 


\section{Community level stress indicators - Optical, structure and recovery} indicators
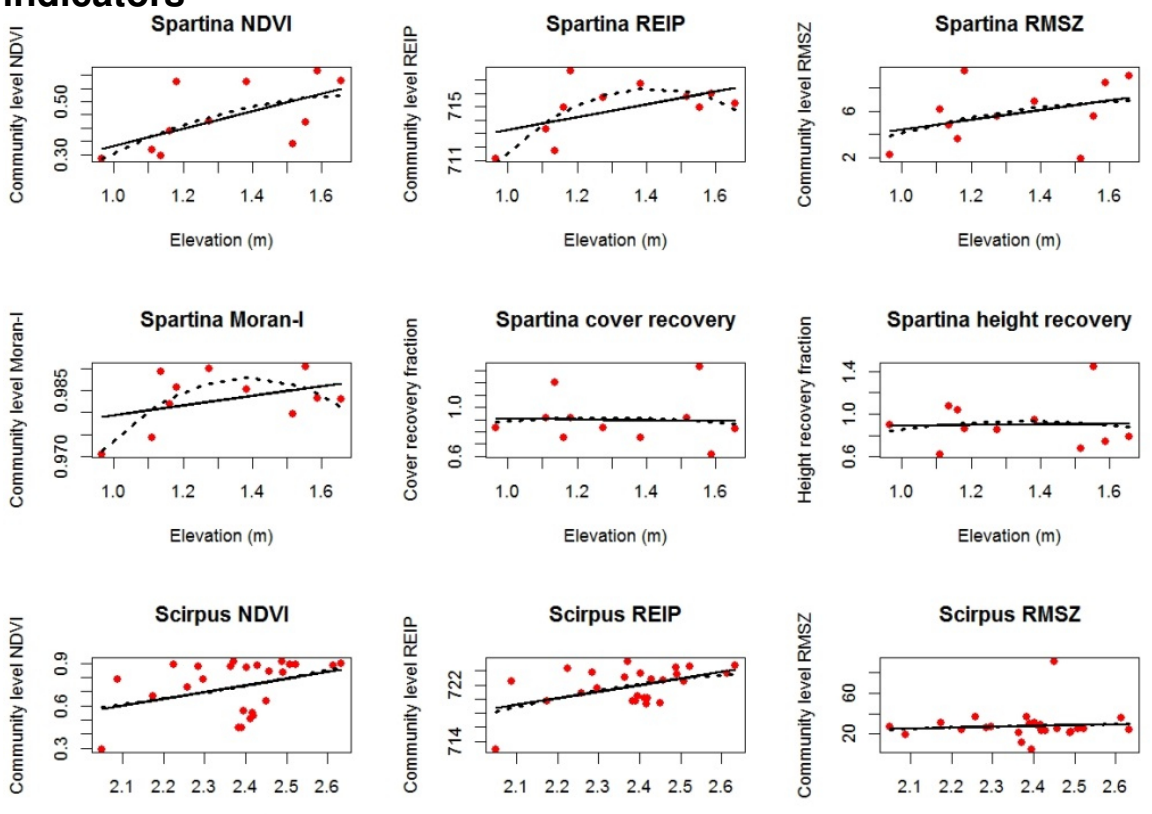

Elevation $(m)$

Elevation $(m)$

Elevation (m)
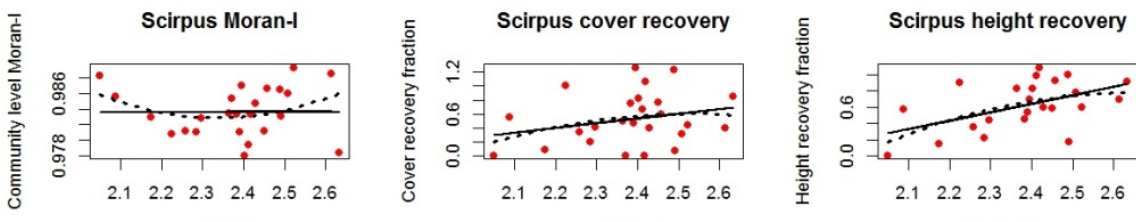

Elevation $(m)$

Elevation $(m)$

Elevation $(m)$

Figure 5.5. Relation of the community level stress indicators with elevation. The solid line is the linear model, the dotted line represents the quadratic model. Recovery fractions is the fraction recovered relative to the corresponding control after 11 months. 


\subsection{Discussion}

Our aim was to investigate which stress indicators are sensitive to stressors along an elevation gradient, and may therefore be used to monitor system stability in saltmarshes. We looked at various biophysical saltmarsh vegetation properties at leaf (chlorophyll content, reflectance), plant (cover, biomass, recovery) and community (cover, biomass, height, recovery, reflectance and structure) level. To enable this analysis, biomass is used as a reference-indicator for the stress response. At the plant level, we see that biomass initially increases with elevation, hence at lower elevations plants are stressed. Flood duration, has been shown to have a huge impact on the pioneering zone of salt marshes (Oteman et al. 2019). At lower elevations vegetation suffers from this inundation stress (Visser et al. 2006). At intermediate elevation we see the highest biomass; at higher elevation we see a decrease in biomass. Spartina is known to perform better at higher elevations, but as a pioneering species it cannot compete with other vegetation there (Bertness and Ellison 1987, Bertness and Shumway 1993, Silvestri et al. 2005). This shows that the plants are stressed at both low and high elevation, which is confirmed by the biomass measurements in both Spartina and Scirpus. This means the hypothesized stress pattern along the elevation gradient does indeed occur, and that we can compare the stress indicators with elevation to establish their sensitivity towards different kinds of stress.

\subsubsection{Leaf level}

For Spartina, we found a strong decrease of chlorophyll content at higher elevation (see also: Oteman et al. 2019). This indicates that chlorophyll content is positively affected by inundation stress. A previous study found a decrease in chlorophyll concentration in Spartina as a response to high concentrations of copper (MateosNaranjo et al. 2008), indicating that chlorophyll can be affected by stressors. In Scirpus, the effect of a decrease in chlorophyll with distance away from the seaward saltmarsh edge was less evident, although the chlorophyll content also decreased at higher elevations. A possible explanation for this difference is a slower development at lower elevations. The chlorophyll content of Scirpus is known to decrease after senescence (Duarte et al. 2012), hence if vegetation develops faster at higher elevations, its chlorophyll content might already have start to decrease. However, the $\mathrm{R}^{2}$ showed that especially in Scirpus chlorophyll content explains limited variation. Another possible reason behind this is that the chlorophyll content may be regulated through nutrients. There have been many studies showing decreased chlorophyll due to nutrient imbalance and how this in 
turn can affect reflectance (Ewing et al. 1995, Homolova et al. 2013, Tong and He 2017). It has been suggested that low marsh species can outcompete high marsh species when sufficient nutrients are available (Emery et al. 2001). However, this seems to contradict the notion that species are either stress resistant or competitively strong (Grime 1974). Another possible explanation is that because we studied top leaves, the difference in chlorophyll content along the elevation gradient is due to an adaptation to flooding, plants at lower elevations could concentrate their chlorophyll in top leaves, as these are flooded least. Because it remains unclear what controls the chlorophyll content pattern along the elevation gradient, and because the pattern appears to differ strongly between species, chlorophyll content is unlikely to be a reliable stress indicator in salt marshes.

The leaf-level reflectance analysis showed that the NDVI and REIP are best described by a quadratic polynomial for Spartina. Especially the NDVI shows a clear optimum at intermediate elevations, and hence can be an indicator of both hydrodynamic and competition stress in Spartina. Scirpus showed a different pattern, it showed an increase in NDVI with elevation. Hence the stress effect of inundation was clearly visible, but a negative effect of competition was not seen in the leaf level reflectance for Scirpus. The reflectance of Scirpus shows more variation in general, with much higher NDVI values, indicating it is likely saturated (cf. detailed spectral profile of Scirpus in Schmidt and Skidmore 2003). In this study we focused on the NDVI as it is an often used index, but many other indices are available that are less susceptible to saturation (Mutanga and Skidmore 2004), but these all also come with limitations. Leaf level reflectance corresponds well with stressors, and shows that these stressors affect plant biochemistry, instead of only altering plant cover. However, leaf level reflectance is nevertheless not very practical as indicator, because sampling reflectance at the leaf level is labor intensive, and the effectiveness differs between species.

\subsubsection{Plant level}

At the plant level we looked at biomass (as reference), cover and cover recovery as indicators of stress.

Vegetation cover estimates are less closely linked with elevation than biomass, but relations are also highly significant in both species. Vegetation cover has been used as a status indicator in many systems for many years (Van der Maarel 1971, Ellenberg et al. 1991), even in salt marshes, where it was used to study effects of competition and facilitation (Bertness and Shumway 1993). 
The analysis of recovery along an elevation gradient showed a stronger recovery at higher elevations for both Spartina and Scirpus. It should be kept in mind that recovery is calculated as a fraction of the cover in the corresponding control plot, and therefore the relatively high recovery rates at higher elevation are partially caused by a low cover in the corresponding control plots. This indicates that these pioneer species are able to quickly recover after a disturbance. Although plants were higher at higher elevations, the pattern of Spartina indicates a large amount of variation. Vegetation may have been already recovered beyond the point where effects were visible, although many plots still had lower cover than their corresponding control (for a complete discussion on recovery in marine systems see: Soissons et al. 2014, 2016). A previous study (Bertness and Shumway 1993), suggested recovery of Spartina can take multiple years although in addition to cutting away above ground biomass they also applied a herbicide in addition to removing all above ground biomass.

Scirpus showed a linear increase of recovery rates towards higher elevations, even when only the mono-specific plots were considered. In these high-elevation plots the cover in the control plots was highest, and therefore this cannot be an effect of quick recovery due to a low initial cover.

The plant level indicators biomass and cover showed the expected response to both inundation and competition stress. Both appear highly suitable as stress indicators. The recovery rate of vegetation cover differs strongly between species and cannot easily be interpreted.

\subsubsection{Community level}

At the community level, we looked at vegetation height, reflectance, structure and cover and height recovery along the elevation gradient. For both study species, the patterns at the community level mostly show a linear increase with elevation, as expected. In the community analysis only the mono-specific plots are taken into account to avoid interference by other species, therefore at this level we can only analyze the effect of hydrodynamic forcing.

Vegetation height relates well with elevation in Scirpus (i.e., low values at low elevations), but is not significant in Spartina, this exemplifies that height as stress indicator strongly depends on species. Height recovery shows a similar pattern, it performs well in Scirpus and relatively poorly in Spartina. A previous study in North America into Spartina height recovery after disturbance showed a quick recovery (Lindau et al. 1999), they found no significant height differences 15 weeks after disturbance (all above ground biomass was burned). It is therefore possible that height recovery has to be measured relatively quickly after disturbance, depending 
on the study species. The large difference between the two pioneering species indicates that height and height recovery are unsuitable as general stress indicator. Although they could still be used in agricultural systems, which often consist of a single species for which height and height development are well defined. In these systems height is known to relate to stress (Cakir 2004, Anjum et al. 2017).

The NDVI, which could be expected to be a function of biomass and cover, only performs well in Spartina (with the lowest values at the lowest elevation). In Scripus the NDVI seems to be saturated, as it was at the leaf level. NDVI is known to saturate at high canopy densities (Mutanga and Skidmore 2004). In such cases using another index is more appropriate (Mutanga and Skidmore 2004). For example, the REIP performed well for both species. The major advantage of a reflectance-based approach is the scalability, allowing for it to be used in combination with a space borne platform, albeit typically at community level only.

The processing of vegetation structure strongly depends upon the statistical measure applied. Hence, we compared the RMSz and Moran's-I, showing that only the latter responded to elevation in Spartina vegetation. Although vegetation structure is important when assessing inundation stress, it is clear that further study into vegetation structure statistics is needed to come to a generalized measure applicable to a wide variety of species.

\subsubsection{Recovery}

Originally recovery rate was proposed as a warning system to predict critical transitions, because regular stress indicators cannot be used to predict these sudden shifts (Scheffer et al. 2009). It was later argued that even without a potential critical shift recovery rate can be used as a general tool to analyze system health (Kéfi et al. 2013). Recently recovery rate was shown to significantly depend upon inundation stress in salt marshes (van Belzen et al. 2017). We therefore expected the recovery rate to relate well with the elevation gradient. However, we found large differences between species and a relatively poor overall relation with the elevation gradient. This could be explained by differences in disturbance technique, seasonal timing of the disturbance or the subsequent monitoring (for a complete discussion on this see: Soissons et al. 2014, 2016). However, this indicates this technique is very sensitive and cannot directly be applied to establish ecosystem stability on a large scale. It should be noted that critical slowing down was originally introduced as a stability measure of bifurcated systems and it may well be the only 
measure that quantifies the resilience towards a shift to an alternate stable state. In this study we only focused on stress indicators, and short-term recovery rates appeared too variable over the season to be used as stress general indicator.

\subsubsection{Conclusion and application to other species}

We analyzed the response of leaf, plant and community traits to elevation in two similar species (i.e., both pioneering lower marsh grasses), and nevertheless found large differences between species. The validation of the indicators using a second species is very valuable, as many stress indicators performed well in one species and not in another. This cautions against extrapolation to other species without additional validation. Although leaf-level pigment and reflectance analyses work reasonably well in both species, we do not recommend them for general stability monitoring as they would require large sampling efforts and likely would not provide uniquely insightful data. At the plant and community level biomass, cover and to some extent reflectance yield consistent results, so that they can be recommended for application to other species. For detailed stress measurements, physical measurements such biomass or cover seems best. Therefore we recommend using phytosociology combined with biomass measurements for monitoring highly vulnerable areas or areas that provide critical ecosystem services. In many situations this is not feasible as it requires intensive sampling campaigns. In such situations, we recommend monitoring based on remote sensing, combined with area specific calibration.

Indices such as the NDVI are often used as indicator, but may not give the best performance in dense vegetation (Mutanga and Skidmore 2004). The major advantage of using such an index is its scalability; space borne remote sensing can be used to quickly and easily collect data. Models that use satellite data to calculate vegetation cover and biomass are plentiful (for a selection for coastal ecosystem see Hickey et al. 2018, Zhou et al. 2018, Eon et al. 2019). However, our results support the notion that the choice of which reflectance index or model is used is not trivial. Aspects such as the height of the vegetation and Leaf Area Index, and hence its sensitivity to saturation should be taken into account. In our two study species the REIP performed well, but the large differences between studied species make clear that prior to application to another species, this should be further validated. 


\section{Chapter 6: Synthesis}

Salt marshes are important ecosystems, they provide numerous vital ecosystem services (see chapter 1). Monitoring an ecosystem service by monitoring the species that provide that service can be deceptive as a natural ecosystem has many interdependencies. To safeguard these services we should therefore aim to monitor the entire system, including large shifts to other vegetation types. For example, for one of the major ecosystem services provided by salt marshes, wave mitigation (reducing wave strength) (Möller 2006, Barbier et al. 2008, Koch et al. 2009, Morgan et al. 2009, Chmura 2011, de Groot et al. 2011), the vegetation type seems unimportant. However, because the pioneering zone protects and facilitates the higher marsh (Bertness and Shumway 1993), a disappearance of the pioneering zone might have rapid detrimental effects on the high marsh. In this study we focus mainly on developing tools to monitor the pioneering zone, which is the most dynamic vegetated zone in a salt marsh, and thereby likely the most difficult to monitor. However, we argue that this should always be part of a broader system monitoring, where the entire systems integrity is taken into account.

A system can change through (anthropogenic) stochastic events, these changes are inherently hard to predict due to their stochastic nature. In this study we focus on non-stochastic changes that involve an increase in vegetation stress prior to changing. We therefore look at several ways to measure vegetation stress in salt marshes. We try to link these vegetation measures to their underlying drivers. Overall, we work towards a remote sensing based monitoring system, as these saltmarsh systems are remote and typically poorly accessible. In this thesis I aim to answer 'how can we establish efficient stress indicators to monitor ecosystem service stability in European coastal marshes, to help safeguard vital ecosystem services'. To achieve this goal, I addressed these four sub questions:

1. How to include high-detail in situ structure measurements in our stress indicator assessment?

2. How do the major stressors in salt marshes, affect vegetation properties and how do these properties affect reflectance?

3. Can we use satellite remote sensing data to establish an indicator for reed development?

4. How well do potential stress indicators, including recovery rate, represent environmental and competition stress? 
In ecology, using vegetation stress to determine ecosystem service stability is complex, partially because stress can act on several different levels. Therefore, part of understanding vegetation stress is understanding:

- which stress indicator performs well on a particular spatial scale

- how this translates to other spatial scales.

Hence, we examined stress indicators at three different levels, 1) leaf level, 2) plant level and 3) community level.

Saltmarshes monitoring in the field may be challenging as salt marshes are regularly flooded, and their creeks may be hard to traverse. This, together with the increasing quality of remote sensing data, and their decreasing costs, make satellite data potentially appealing for salt marsh monitoring. Although new technologies are continuously being developed, most remote sensing data can be divided into two groups:

A) Radar based data, which, for active sensors, typically concern the backscatter return relative to emitted microwave pulses, particularly depending on the structure of the object under observation, and its dielectric properties. The used radar wavelength has a large influence when observing vegetation.

B) Optical data, which, for passive sensors, typically concern the reflected (and absorbed and transmitted) sun light, relative to emitted sunlight in a number of wavelengths. These optical data can be analyzed by calculating a Vegetation Index (VI).

We will now first discuss the vegetation structure as a potential stress indicator (as detected with "depth from focus" using optical images and with radar remote sensing), then we will discuss the physical stress indicators and their corresponding vegetation indices (as detected using optical remote sensing techniques). Following that, we discuss the potential for resilience based measurements as stress and stability indicator. Then we discuss how indicators should be applied. Finally, we discuss the societal benefits of this study and give our recommendations for further study. 


\subsection{Vegetation structure as stress indicator}

Vegetation structure plays a vital role in ecosystems, it has a strong effect on many trophic interactions (Joern 1982, Gunnarsson 1990, Martín and López 1998, Brose 2003, Obermaier et al. 2008, Cho et al. 2017). In salt marshes vegetation structure is directly related to one of the most important ecosystem services, flood protection through wave mitigation (Bouma et al. 2005, Möller 2006). We measured structure in situ at the community level using a newly developed technique named depth from focus (chapter 2). This answers our first research question, 'How to include high-detail in situ structure measurements in our stress indicator assessment?'. The depth from focus technique was used to create detailed $3 \mathrm{~d}$ vegetation models from optical camera images obtained in situ, from these models we derived four spatial statistics (i.e., RMSz, Spatial autocorrelation, Tortuosity and Moran's-I). Linking these directly to in situ vegetation stress yielded no stress indicators that were consistent between species (partially answering the fourth research question: 'How well do potential stress indicators, including recovery rate, represent environmental and competition stress?'). Although often used and well established (Moran 1950, Bretar et al. 2013) these four spatial statistics might not be the most appropriate as indicators for stress. Further study into which statistics are most suitable to derive vegetation stress from $3 \mathrm{~d}$ images is recommended.

Vegetation structure could also be obtained from space borne radar data. A major challenge in Synthetic Aparture Radar (SAR) satellite remote sensing is that many factors affect the signal. Especially water content is known to be important in radar backscatter (Hill et al. 1999), exemplified by the often used water cloud model (Graham and Harris 2003). In this model the vegetation is assumed to be a cloud of water, and the vegetation is reduced to its height and its water content. However, vegetation is known to be more complex, and the study of which parts contribute, and how deep radar can penetrate the vegetation layer is ongoing. The penetration depth also depends on the radar wavelength. Currently this is studied using radar reflectors (Algafsh et al. 2017) or by using seasonal change in the response to surface soil moisture content (El Hajj et al. 2019). The application of in situ structure measurements, combined with SAR data could potentially provide insight into how deep the SAR microwave beams penetrate the vegetation canopy. A better understanding of the penetration depth would help us understand the extent of the canopy contribution to the overall backscatter, which would help us better interpret backscatter data.

The application of structure analyses on a large scale in the Romanian reed lands showed that vegetation structure is an important predictor of vegetation development. We found that although optical data did not represent system 
stability well, vegetation structure, and vegetation structure development related well with stability. We found that in expanding reeds, the seasonal difference in $\mathrm{C}$ band SAR-backscatter were much larger than in areas where reed was retreating. The stable situation showed intermediate backscatter. The underlying cause behind these differences in radar backscatter remains unknown, and a link with plant or leaf level data could not be made based on the collected dataset. In vegetation studies, radar backscatter is generally accepted to represent vegetation structure (for example see: (Imhoff et al. 1997, Lou et al. 2017, Jagdhuber et al. 2020). That vegetation structure relates to vegetation stress in reed lands seems obvious. Reed lands are generally dominated by a single species, and have a distinct pattern of numerous stems standing up straight, with leaves mixed in at various heights. This pattern is affected when the plants are stressed. We suspect that in other systems that contain more species a similar pattern can be found.

Most coastal systems have low species diversity, only a handful of specialized species occur, and our analyses showed several stress responses that will likely affect vegetation structure (see chapter 5 for several examples). We therefore argue that the conclusions in chapter 4 are likely to occur in other (nonmonospecific) coastal ecosystems as well. For example, most borders between vegetation types in many salt marshes are sharp, broad diffuse borders are less common. These sharp borders imply fierce competition, which has been reported to shift under influence of elevation changes (Bertness and Ellison 1987, Silvestri et al. 2005). This stressed vegetation state implies vegetation structure will likely be affected. For example, our in situ measurements in saltmarshes showed a decrease of vegetation cover of pioneering species, but field observations indicate the total vegetation cover also decreased in areas where multiple species occur (chapter 5). Therefore vegetation stress on these vegetation borders might also be detectable using radar. It should however be taken into account that as these borders are sharp, their effects might be narrow and hence a high signal to noise ratio as well a high spatial resolution may be required to detect it.

In non-coastal vegetation types such as grasslands or heathlands, the possibility of using structure as an indicator for stress seems less obvious; these systems potentially consist of many species with varying degrees of allospecific competition. In general we expect structure to be a good stress indicator in situations with strong intraspecific competition, such as reedlands or other monocultures, where allospecific competition stress only occurs in the borders. For other types of stress, it will strongly depend upon how that specific stressor affects vegetation structure. In salt marshes monitoring stress through structure has the 
additional advantage of being directly related to several primary ecosystem services (flood protection and wave mitigation).

\subsection{Physical stress indicators and corresponding vegetation indices}

We analyzed physical stress indicators at the leaf and community level. The first indicator we examined is the pigments: chlorophyll $a$, chlorophyll $b$ and carotene. These are directly related to the photosynthetic capacity of vegetation. It is known that stress can be detected by measuring chlorophyll content (Carter and Knapp 2001b, Zarco-Tejada et al. 2002). Pigments are often used as a response variable for Spartina stress (Sun et al. 2018), and a dedicated Chlorophyll Vegetation Index (CVI) is available (Vincini et al. 2008). In our study, in situ measurements of chlorophyll of Spartina leaves showed a strong response of chlorophyll content to inundation stress, but a simulation using the radiative transfer model PROSAIL (Jacquemoud et al. 2009) showed that the effect of these pigments on reflectance is negligible along the inundation gradient of the saltmarsh (see chapter 3 ). There are many studies where pigments do affect reflectance substantially, and several indices specific to estimating pigment content are available (Roberts et al. 2016). In our study area the differences between pigment contents had only a minimal effect on reflectance, making it very unlikely that stress can be modelled through pigments using space borne data in our study area. It is important to note that in other, mostly agricultural, systems, specialized models and calibration datasets are available. Which makes it possible to detect pigments or nutrient contents (Gitelson and Merzlyak 1996, Kooistra and Clevers 2016, Lawley et al. 2016). For salt marshes these models will have to be adapted and further calibration will be required. As we found very little response of chlorophyll in PROSAIL, developing such a model for salt marshes will likely not be an easy task.

Many reflectance indices, such as NDVI or SAVI, aim to estimate above ground biomass as stress indicator (Campbell and Wang 2020). Based on previous research (Bertness et al. 1992, Bertness and Hacker 1994) we assumed salt marsh pioneers would be stressed both at high and low elevations by competition and flood related stress, and hence we expected an optimum in biomass at intermediate elevations. We found the relation between biomass and elevation to indeed represent the expected pattern, therefore modelling biomass is likely to provide a solid basis for a reflectance based stress indicator. We measured reflectance at the leaf and community level. We measured two different species, 
to gain insight into inter species variability. At the leaf level we saw NDVI representing inundation stress reasonably well for both species, in one of the studied species (Spartina) it also represented competition stress (chapter 5). For this species especially, the NDVI at leaf level followed a similar pattern as biomass of the vegetation. At leaf level the REIP (red edge inflection point) showed much variation and was not significantly related to flood duration or competition.

At the community level, with reflectance measured at $2 \mathrm{~m}$ height, we found the reflectance-based index REIP to perform better than NDVI in both studies species. This indicates that when choosing a vegetation index, the measurement level is crucial; an index that relates well to stress at the leaf level might not relate well to stress when applied at the community level. Our community level in situ measurements show that NDVI is vulnerable to saturation (chapter 5), this was also indicated by our experiment in Romania (chapter 4) and has been previously described (Mutanga and Skidmore 2004). SAVI is known to be less likely to saturate (Campbell and Wang 2020), and has been shown to work well in reed lands (Poulin et al. 2010). However, when analyzing reed stability (chapter 4), we found that optical vegetation indices related poorly to the expansion and retraction pattern and the corresponding expected stress. In this case radar data related much better with the development patterns in reeds (chapter 4). This indicates that analyzing stress is very much a system specific process. A single spectral index or tool cannot be applied to all situations without targeted calibration and preferably an understanding of the underlying processes.

To gain a better understanding of the major shaping processes in salt marshes, and hence likely underlying stressors, we applied PROSAIL (Jacquemoud et al. 2009) to salt marsh vegetation (chapter 3 ), which showed that especially Leaf Area Index (LAI) contributes strongly to variations in reflectance in our study system. LAI is known to be closely related to biomass. This is the answer to the second part of our second research question 'How do the major stressors in salt marshes, affect vegetation properties and how do these properties affect reflectance?'. The multitude of spectral indices focused on biomass will likely produce useful results in our study system. This does introduce the assumption that whatever is causing stress affects biomass. The first part of the second research question was also answered in chapter 3 , where we concluded that the strongest stressor in our study system was flood duration. However, in this chapter we did not analyze the effects of direct competition on reflectance, to exclude mixel effects. Therefore, the results of chapter 3 do not contradict the results of chapter 5 , where we showed that both competition and flood duration have a strong effect on biomass. These strong effects on biomass indicate that biomass based reflectance indices will likely perform well here. Our simulations based on the PROSAIL model (chapter 3) 
indicated the most important factor for Spartina reflectance in our study system is $\mathrm{LAl} /$ biomass. Our in situ measurements in chapter 5 confirmed this, as NDVI related well with the measured stress patterns (through biomass) for Spartina. However, when applied to another species, Scirpus (also chapter 5), NDVI was found to saturate quickly when biomass increased. This does not indicate that biomass is not important, but that NDVI should not be used to model it; another vegetation index e.g. REIP (Red Edge Inflection Point) did represent stress well. In our Romanian study site, in situ biomass measurements showed no significant relation to season, and related poorly with expected stressors. It is therefore not surprising that optical remote sensing related poorly with stress (chapter 4). This allows us to answer our third research question 'Can we use satellite remote sensing data to establish an indicator for reed development?'. Our results indicate biomass based vegetation indices can provide useful information, our results also show that stress detection is likely highly area specific, and in the choice of the appropriate stress indicator, the effect of expected stressors on reflectance cannot be ignored.

When analyzing stress, it seems important to first answer the question, 'how will this stressor affect the vegetation'. If a direct effect on biomass is expected, reflectance will likely be a suitable stress indictor. Else other measurements will likely be more appropriate. This is part of the answer to our fourth research question, 'How well do potential stress indicators, including recovery rate, represent environmental and competition stress?'. Stress can be modelled using vegetation indices, but the choice of index should depend on the target species, the expected effect (is biomass affected) and the measurement level (leaf, plant or community). 


\subsection{Resilience as stress indicator}

In chapter 1 we proposed a thought experiment to help explain how plants are able to reduce their total stress by exchanging one stressor for another. In some cases feedbacks might even allow a plant to reduce stress with minimal increase to other stress factors. There is a limit to these feedbacks, when the conditions (the habitat point, see chapter 1) move too much, a different vegetation state can gain the upper hand and a sudden shift occurs. If other feedbacks pull the habitat point to a new equilibrium we speak of alternate stable states, this concept was already discussed by DeAngelis et al. (1989) but made more popular by (Scheffer et al. $2001,2009)$. Because these feedbacks allow plants to mitigate the effects of a severe stressor at the cost of another, the effect of the severe stressor can remain hidden until the transition to an alternate state. There are many examples of plants that lower or raise $\mathrm{pH}$ to improve their competitive position, even though it might not improve their growing conditions.

For the salt marsh species we studied, there is no indication they actively 'sabotage' their environment to increase their competitive position. In fact facilitation is an often mentioned process in salt marshes (Bertness and Shumway 1993), where one species makes the habitat suitable enough for another to take over. This is likely because the high dynamics already present enough stressors to have different niches. At the seaward edge of these systems establishment of new vegetation is difficult (Bertness and Shumway 1993), under these harsh conditions establishment almost always requires multiple individuals to support each other (Bertness and Shumway 1993). This creates a strongly feedback dependent system, once the first plant is established, establishment for others becomes easier. If during a storm most of the plants die, the remainder is less likely to survive. This creates the interesting situation of having a strongly feedback dependent stressor seaward, and a non-feedback dependent stressor landward. In the feedback dependent system we may expect a more binary or steep sigmoid transition curve between to states (vegetated or not vegetated). In the nonfeedback dependent system higher in the marsh, where multiple species are in competition, we may expect a more linear transition. This means that the chance of a plant disappearing is inversely related to its cover.

Traditionally it is assumed that feedback dependent processes cannot be measured by biomass, as biomass development generally has a high priority for plants. These feedbacks allow a plant to develop biomass even close to tipping points. Therefore, biomass is likely most effective as an indicator in linear systems, and is less effective in feedback dependent systems. However, in chapter 4 we showed a strong response of biomass to flooding stress, which is a feedback 
dependent process. Previous studies (van Belzen et al. 2017) also show they are able to model this feedback dependent stressor using biomass as a stress indicator. Therefore we argue that it depends on the stressor if it can be monitored using biomass as indicator. In the case of salt marshes, biomass is directly related to the strength of the feedback (i.e. more plants means less stress). It is important to note that although biomass is directly affected, it will likely still have a steeper transition curve than in the linear situation. It has been argued that resilience (recovery rate after disturbance) should be used to measure stability (van Nes and Scheffer 2007, Veraart et al. 2012) rather than biomass or phytosociology, and that these measurements could also provide insight into systems without alternate stable states (Kéfi et al. 2013). We found little evidence to support this, and showed that resilience measurements differed largely between species and were difficult to interpret.

This insight into recovery rate is the final piece of information to answer the fourth research question ('How well do potential stress indicators, including recovery rate, represent environmental and competition stress?'). During our data collection for chapter 5, we noticed an interesting phenomenon regarding the quality of vegetation recovery. Although several plots seemed to have completely recovered before winter, in spring the recovery was incomplete. This is likely because the winter storms had a larger impact on plots that received a disturbance treatment before. This implies that quality of recovered vegetation potentially plays a large role, as although the biomass had recovered, it was not of sufficient quality to withstand perturbations. This has large implications for when recovery can be measured; measuring recovery before winter would have indicated a higher resilience than measuring it in spring. This is because this type of resilience measurement is a combination of the initial resilience and the additional stressors in the recovery period (this type of measurement can contain both the recovery of biomass, and the recovery of resilience). It could therefore be argued that a time series is required to understand resilience.

This argues for caution against using resilience measurements as general indicator, as the process of resilience measurement is still not completely understood. Similar responses were found when applying resilience measurements in sea grasses (Soissons et al. 2014, 2016). Although we argue that resilience needs to be developed further before it can be used as a reliable stress or stability indicator, the idea of flickering prior to transition (Dakos et al. 2013), or using the rate of change as indicator of system stability, was shown to be useful in some cases (chapter 4). 


\subsection{Societal benefit and applications}

The methods developed in this thesis may have broader applications and can support further studies in many ways. Our new structure analysis tool (depth from focus, see chapter 2) to quantify vegetation structure could be widely used and be of great benefit to many areas of study, for example to help protect flying insects, a group known to be under great pressure (Hallmann et al. 2017) and crucial to a well-functioning ecosystem. In salt marshes we expect this technique can make great contributions to wave-attenuation modelling efforts and be used to gain more insight into the penetration depth of radar in salt marsh vegetation canopies.

Another major societal benefit of this study is the potential for improved coastal management. In chapter 3 we analyzed what major stressors are in salt marshes and how these affect reflectance. This helps us better understand and thereby potentially manage our salt marshes. For example this analysis showed that flood duration is the most important driver behind the large scale patterns in vegetation properties in salt marshes (see chapter 3). Therefore, sudden changes in flood duration, potentially caused by changes in elevation or sea level, could drastically change these large scale patterns in salt marshes.

Another management benefit of this study comes from chapter 4, where we use remote sensing to analyze a huge reed area in Romania. Here reed provides many ecosystem services, but its development status was previously unknown. We not only found out that the reed lands are expanding, but also provided a tool to help predict where expansion and retraction would take place. This will help safeguard the ecosystem services provided by this system.

We provided a start of a similar analysis for more complex systems, by modelling what the effects are of biophysical properties (chapter 3 ) and stress (chapter 5) on reflectance. If further developed this could provide a similar safeguard of the ecosystem services provided by multispecies systems such as the Dutch salt marshes. 


\subsection{Outlook for further study}

We would first recommend a continuation of the radar studies. Understanding what backscatter represents in terms of vegetation structure in saltmarshes would be highly valuable in interpreting SAR satellite images. With the optical depth-fromfocus technique developed in chapter 2 it should now be possible to establish how deep radar penetrates into the vegetation canopy, and retrieve metrics for vegetation structure in coastal ecosystems.

Another important aspect that needs to be studied further specifically for salt marshes, is the sensitivity of vegetation indices to biomass and the sensitivity of these indices to other vegetation properties such as resilience. The feedback systems that allow alleviation of specific stressors likely enforce other stressors that may cause smaller problems. However, moving the habitat conditions away from the optimum for a plant will always cause an increase in stress. If we can measure these smaller stressors using highly detailed in situ spectral measurements we might be able to use reflectance to establish stress even in situations where feedbacks would normally make stress measurements difficult. This could be established by measuring a wide variety of vegetation properties together with reflectance along a stress gradient, and then rather than calculating predefined vegetation indices, calculating a wide range of indices to establish which wavelengths and wavelength ratios correspond to specific properties. Once this is established a high resolution multi- or hyperspectral satellite image (with a resolution of $50 \times 50 \mathrm{~cm}$ or smaller) could be used to compare in situ measurements with remote sensing data. This would help assess if competition and inundation stress can be distinguished in salt marshes using remote sensing.

Due to the complex nature of ecosystems, it will be difficult to establish which species help provide an ecosystem service. Therefore the system should be monitored as a whole. In this study we solely focus on the pioneering zone of salt marshes, and only studied a few species in depth. We strongly recommend that our work be expanded to include other species, and the other parts of coastal ecosystems. As only with broad monitoring can we effectively monitor the stability of the ecosystems services provided by these systems.

During these studies we developed many ideas that were not fully tested. One of them was the possibility to use Ellenberg indicators to predict where shifts in vegetation composition are likely to occur. Ellenberg (Ellenberg et al. 1991) proposed that by looking at the tolerance range of a species, the habitat conditions could be derived with respect to aspects such as $\mathrm{pH}$, salinity and water availability. Later this system was expanded by others such as Wamelink (Wamelink et al. 2005). These indicator systems can be applied to vegetation maps, potentially even if these are derived from remote sensing. Several studies have shown 
indicator values can also be directly derived from remote sensing data (Schmidtlein 2005, Besnard et al. 2015, Möckel et al. 2016). We propose a landscape analysis to locate borders between species that have large differences in their indicator values. These borders are then expected to be less stable, and hence more likely to shift, than borders of more similar species. Further research is needed to test this theory, and evaluate how sensitive these indicators are, to detect vegetation shifts. 


\subsection{Conclusions and recommendations}

In this study we aim to support stress monitoring in salt marshes to aid management. We found that there is no single vegetation index that is likely to represent stress well in all salt marshes. In Spartina dominated saltmarsh, we found NDVI to perform best, while for a similar species (Scirpus) we found REIP to perform better. Our results even suggest vegetation indices may have to be supported by radar analysis in some situations (e.g. Phragmites), as structure information may hold information not visible through optical data sources. Therefore, stress monitoring will have to remain a very area specific task, and although remote sensing will be a valuable tool for these analyses, it will have to be applied by experts who understand the caveats of techniques and the limitations of a specific vegetation index.

We like to plea for an increased scientific usage of vegetation structure data, both in remote sensing and in situ. Further development of these techniques and an increased understanding of these techniques are required before they can be applied to support management. We would argue for similar caution when applying recovery rate to estimate system stability or system stress.

To salt marsh managers we would therefore recommend:

- Use remote sensing to monitor stress development in salt marshes, but be sure to have a remote sensing expert and ecologist involved to ensure proper usage of vegetation indices and their interpretation.

- Biomass is still a reliable way to establish stress, however due to its labor intensive nature, it should be reserved for monitoring very vulnerable or critically important systems. Note that potential damage to the system should be taken into account

- Aim to monitor the system as a whole, and include shifts in vegetation type. And scientists we would encourage to:

- Support managers by helping to interpret remote sensing data

- Further explore how vegetation structure can be incorporated into monitoring schemes as a stress indicator

- Further validate recovery rate as potential tool to estimate stress in salt marshes

- Continue to develop the tools we presented here, expand them to include other sections of the salt marsh to allow for broad ecosystem monitoring. 


\section{Bibliography}

Aldakheel, Y. Y. 2011. Assessing NDVI spatial pattern as related to irrigation and soil salinity management in Al-Hassa Oasis, Saudi Arabia. Journal of the indian Society of Remote Sensing 39:171-180.

Algafsh, A., M. Inggs, and A. K. Mishra. 2017. Measurements of signal penetration for p-band SAR system through trees using two trihedral corner reflectors. Pages 3117-31202017 IEEE International Geoscience and Remote Sensing Symposium (IGARSS). IEEE.

Ali, I., F. Cawkwell, E. Dwyer, B. Barrett, and S. Green. 2016. Satellite remote sensing of grasslands: from observation to management. Journal of Plant Ecology 9:649-671.

Anderson, K., S. Hancock, M. Disney, and K. J. Gaston. 2015. Is waveform worth it? A comparison of LiDAR approaches for vegetation and landscape characterization. Remote Sensing in Ecology and Conservation.

Anjum, S. A., U. Ashraf, A. Zohaib, M. Tanveer, M. Naeem, I. Ali, T. Tabassum, and U. Nazir. 2017. Growth and development responses of crop plants under drought stress: a review. Zemdirbyste 104:267-276.

Baghdadi, N., M. Bernier, R. Gauthier, and I. Neeson. 2001. Evaluation of C-band SAR data for wetlands mapping. International Journal of Remote Sensing 22:71-88.

Barbier, E. B., E. W. Koch, B. R. Silliman, S. D. Hacker, E. Wolanski, J. Primavera, E. F. Granek, S. Polasky, S. Aswani, and L. A. Cramer. 2008. Coastal ecosystem-based management with nonlinear ecological functions and values. science 319:321-323.

Baret, F., and G. Guyot. 1991. Potentials and limits of vegetation indices for LAI and APAR assessment. Remote sensing of environment 35:161-173.

Barkman, J. J. 1979. The investigation of vegetation texture and structure. Page Werger, M. J. A.(ed.) The study of vegetation. Junk, The Hague,.

van Beijma, S., A. Comber, and A. Lamb. 2014. Random forest classification of salt marsh vegetation habitats using quad-polarimetric airborne SAR, elevation and optical RS data. Remote Sensing of Environment 149:118-129.

Beleites, C. 2017. HyperSpec.

Belluco, E., M. Camuffo, S. Ferrari, L. Modenese, S. Silvestri, A. Marani, and M. Marani. 2006. Mapping salt-marsh vegetation by multispectral and hyperspectral remote sensing. Remote Sensing of Environment 105:54-67.

van Belzen, J., J. van de Koppel, M. L. Kirwan, D. van der Wal, P. M. J. Herman, V. Dakos, S. Kéfi, M. Scheffer, G. R. Guntenspergen, and T. J. Bouma. 2017. Vegetation recovery in tidal marshes reveals critical slowing down under increased inundation. Nature Communications 8:ncomms15811.

Berger, K., C. Atzberger, M. Danner, G. D’Urso, W. Mauser, F. Vuolo, and T. Hank. 2018. Evaluation of the PROSAIL model capabilities for future hyperspectral model environments: a review study. Remote Sensing 10:85.

Bertness, M. D., and A. M. Ellison. 1987. Determinants of pattern in a New England salt marsh plant community. Ecological Monographs:129-147.

Bertness, M. D., L. Gough, and S. W. Shumway. 1992. Salt tolerances and the distribution of fugitive salt marsh plants. Ecology:1842-1851.

Bertness, M. D., and S. D. Hacker. 1994. Physical stress and positive associations among marsh plants. American Naturalist:363-372.

Bertness, M. D., and S. W. Shumway. 1993. Competition and facilitation in marsh plants. American Naturalist:718-724.

Besnard, A. G., A. Davranche, S. Maugenest, J.-B. Bouzillé, A. Vian, and J. Secondi. 2015. Vegetation maps based on remote sensing are informative predictors of habitat selection of 
grassland birds across a wetness gradient. Ecological Indicators 58:47-54.

Bicheron, P., and M. Leroy. 1999. A method of biophysical parameter retrieval at global scale by inversion of a vegetation reflectance model. Remote sensing of Environment 67:251-266.

Boesch, D. F., and R. E. Turner. 1984. Dependence of fishery species on salt marshes: the role of food and refuge. Estuaries 7:460-468.

Botha, E. J., B. Leblon, B. Zebarth, and J. Watmough. 2007. Non-destructive estimation of potato leaf chlorophyll from canopy hyperspectral reflectance using the inverted PROSAIL model. International Journal of Applied Earth Observation and Geoinformation 9:360-374.

Bouma, T. J., M. B. De Vries, and P. M. J. Herman. 2010. Comparing ecosystem engineering efficiency of two plant species with contrasting growth strategies. Ecology 91:2696-2704.

Bouma, T. J., M. B. De Vries, E. Low, G. Peralta, I. C. vd Tánczos, J. van de Koppel, and P. M. J. Herman. 2005. Trade-offs related to ecosystem engineering: A case study on stiffness of emerging macrophytes. Ecology 86:2187-2199.

Bourgeau-Chavez, L. L., K. P. Kowalski, M. L. C. Mazur, K. A. Scarbrough, R. B. Powell, C. N. Brooks, B. Huberty, L. K. Jenkins, E. C. Banda, and D. M. Galbraith. 2013. Mapping invasive Phragmites australis in the coastal Great Lakes with ALOS PALSAR satellite imagery for decision support. Journal of Great Lakes Research 39:65-77.

Bowyer, P., and F. M. Danson. 2004. Sensitivity of spectral reflectance to variation in live fuel moisture content at leaf and canopy level. Remote Sensing of Environment 92:297-308.

Brecko, J., A. Mathys, W. Dekoninck, M. Leponce, D. VandenSpiegel, and P. Semal. 2014. Focus stacking: Comparing commercial top-end set-ups with a semi-automatic low budget approach. A possible solution for mass digitization of type specimens. ZooKeys 23:1-23.

Bretar, F., M. Arab-Sedze, J. Champion, M. Pierrot-Deseilligny, E. Heggy, and S. Jacquemoud. 2013. An advanced photogrammetric method to measure surface roughness: Application to volcanic terrains in the Piton de la Fournaise, Reunion Island. Remote Sensing of Environment 135:1-11.

Brisco, B., M. Kapfer, T. Hirose, B. Tedford, and J. Liu. 2011. Evaluation of C-band polarization diversity and polarimetry for wetland mapping. Canadian Journal of Remote Sensing 37:8292.

Brix, H. 1999. The European research project on reed die-back and progression (EUREED). Limnologica-Ecology and Management of Inland Waters 29:5-10.

Brose, U. 2003. Bottom-up control of carabid beetle communities in early successional wetlands: mediated by vegetation structure or plant diversity? Oecologia 135:407-413.

Busemeyer, L., D. Mentrup, K. Möller, E. Wunder, K. Alheit, V. Hahn, H. P. Maurer, J. C. Reif, T. Würschum, J. Müller, F. Rahe, and A. Ruckelshausen. 2013. Breedvision - A multi-sensor platform for non-destructive field-based phenotyping in plant breeding. Sensors (Switzerland) 13:2830-2847.

Caccamo, G., L. A. Chisholm, R. A. Bradstock, and M. L. Puotinen. 2011. Assessing the sensitivity of MODIS to monitor drought in high biomass ecosystems. Remote Sensing of Environment 115:2626-2639.

Cakir, R. 2004. Effect of water stress at different development stages on vegetative and reproductive growth of corn. Field Crops Research 89:1-16.

Callaghan, D. P., T. J. Bouma, P. Klaassen, D. Van der Wal, M. J. F. F. Stive, and P. M. J. J. Herman. 2010. Hydrodynamic forcing on salt-marsh development: Distinguishing the relative importance of waves and tidal flows. Estuarine, Coastal and Shelf Science 89:73-88.

Camarretta, N., P. A. Harrison, T. Bailey, B. Potts, A. Lucieer, N. Davidson, and M. Hunt. 2020. Monitoring forest structure to guide adaptive management of forest restoration: a review of remote sensing approaches. New Forests 51:573-596.

Campbell, A. D., and Y. Wang. 2020. Salt marsh monitoring along the mid-Atlantic coast by Google 
Earth Engine enabled time series. PloS one 15:e0229605.

Cao, H., Z. Zhu, R. James, P. M. J. Herman, L. Zhang, L. Yuan, and T. J. Bouma. 2019. Wave effects on seedling establishment of three pioneer marsh species: survival, morphology and biomechanics. Annals of botany.

Carter, G. A., and A. K. Knapp. 2001a. Leaf optical properties in higher plants: linking spectral characteristics to stress and chlorophyll concentration. American Journal of Botany 88:677684.

Carter, G. A., and A. K. Knapp. 2001b. Leaf optical properties in higher plants: linking spectral characteristics to stress and chlorophyll concentration. American journal of botany 88:677684.

Castillo, J. M., L. Fernández-Baco, E. M. Castellanos, C. J. Luque, M. E. Figueroa, and A. J. Davy. 2000. Lower limits of Spartina densiflora and S. maritima in a Mediterranean salt marsh determined by different ecophysiological tolerances. Journal of Ecology 88:801-812.

Cerri, M., R. Sapkota, A. Coppi, V. Ferri, B. Foggi, D. Gigante, L. Lastrucci, R. Selvaggi, R. Venanzoni, M. Nicolaisen, F. Ferranti, and L. Reale. 2017. Oomycete Communities Associated with Reed Die-Back Syndrome. Frontiers in Plant Science 8:1-11.

Chambers, R. M., L. a. Meyerson, and K. Saltonstall. 1999. Expansion of Phragmites australis into tidal wetlands of North America. Aquatic Botany 64:261-273.

Chmura, G. L. 2011. What do we need to assess the sustainability of the tidal salt marsh carbon sink? Ocean \& Coastal Management.

Cho, Y., D. Lee, and S. Bae. 2017. Effects of vegetation structure and human impact on understory honey plant richness: implications for pollinator visitation. Journal of Ecology and Environment 41:2.

Clevers, J., S. M. De Jong, G. F. Epema, F. D. Van Der Meer, W. H. Bakker, A. K. Skidmore, and K. H. Scholte. 2002. Derivation of the red edge index using the MERIS standard band setting. International Journal of Remote Sensing 23:3169-3184.

Collin, A., N. Lambert, and S. Etienne. 2018. Satellite-based salt marsh elevation, vegetation height, and species composition mapping using the superspectral WorldView-3 imagery. International Journal of Remote Sensing 39:5619-5637.

Coops, H., R. Boeters, and H. Smit. 1991. Direct and indirect effects of wave attack on helophytes. Aquatic Botany 41:333-352.

Coops, H., N. Geilen, and G. van der Velde. 1994. Distribution and growth of the helophyte species Phragmites australis and Scirpus lacustris in water depth gradients in relation to wave exposure. Aquatic Botany 48:273-284.

Costanza, R. 2000. Social goals and the valuation of ecosystem services. Ecosystems:4-10.

Costanza, R., R. D’Arge, R. de Groot, S. Farber, M. Grasso, B. Hannon, K. Limburg, S. Naeem, R. V. O'Neill, J. Paruelo, R. G. Raskin, P. Sutton, and M. van den Belt. 1998. The value of the world's ecosystem services and natural capital. Nature 387:253-260.

Dakos, V., E. H. van Nes, and M. Scheffer. 2013. Flickering as an early warning signal. Theoretical ecology 6:309-317.

Van Damme, S., E. Struyf, T. Maris, T. Ysebaert, F. Dehairs, M. Tackx, C. Heip, and P. Meire. 2005. Spatial and temporal patterns of water quality along the estuarine salinity gradient of the Scheldt estuary (Belgium and The Netherlands): results of an integrated monitoring approach. Hydrobiologia 540:29-45.

DeAngelis, D. L., P. J. Mulholland, A. V Palumbo, A. D. Steinman, M. A. Huston, and J. W. Elwood. 1989. Nutrient dynamics and food-web stability. Annual review of ecology and systematics 20:71-95.

Deegan, L. A., J. E. Hughes, and R. A. Rountree. 2002. Salt marsh ecosystem support of marine transient species. Pages 333-365Concepts and controversies in tidal marsh ecology. 
Springer.

Dinu, I., G. Umgiesser, M. Bajo, F. De Pascalis, A. Stănică, C. Pop, R. Dimitriu, I. Nichersu, and A. Constantinescu. 2015. Modelling of the response of the Razelm-Sinoe lagoon system to physical forcing. GeoEcoMarina.

Duan, S.-B., Z.-L. Li, H. Wu, B.-H. Tang, L. Ma, E. Zhao, and C. Li. 2014. Inversion of the PROSAIL model to estimate leaf area index of maize, potato, and sunflower fields from unmanned aerial vehicle hyperspectral data. International Journal of Applied Earth Observation and Geoinformation 26:12-20.

Duarte, B., T. Couto, J. C. Marques, and I. Caçador. 2012. Scirpus maritimus leaf pigment profile and photochemistry during senescence: implications on carbon sequestration. Plant Physiology and Biochemistry 57:238-244.

Ellenberg, H., H. E. Weber, R. Düll, V. Wirth, W. Werner, and D. Paulissen. 1991. Zeigerwerte von Pflanzen in Mitteleuropa: Indicator values of plants in Central Europe. Goltze.

Emery, N. C., P. J. Ewanchuk, and M. D. Bertness. 2001. Competition and salt-marsh plant zonation: stress tolerators may be dominant competitors. Ecology 82:2471-2485.

Eon, R. S., S. Goldsmith, C. M. Bachmann, A. C. Tyler, C. S. Lapszynski, G. P. Badura, D. T. Osgood, and R. Brett. 2019. Retrieval of Salt Marsh Above-Ground Biomass from HighSpatial Resolution Hyperspectral Imagery Using PROSAIL. Remote Sensing 11:1385.

Ewing, K., K. L. McKee, I. A. Mendelssohn, and M. W. Hester. 1995. A comparison of indicators of sub-lethal nutrient stress in the salt marsh grass, Spartina patens. Environmental and Experimental Botany 35:331-343.

Fagherazzi, S., L. Carniello, L. D’Alpaos, and A. Defina. 2006. Critical bifurcation of shallow microtidal landforms in tidal flats and salt marshes. Proceedings of the National Academy of Sciences 103:8337-8341.

Feagin, R. A., M. L. Martinez, G. Mendoza-Gonzalez, and R. Costanza. 2010. Salt marsh zonal migration and ecosystem service change in response to global sea level rise: a case study from an urban region. Ecology and Society 15.

Findlay, S. E. G., S. Dye, and K. A. Kuehn. 2002. Microbial growth and nitrogen retention in litter of Phragmites australis compared to Typha angustifolia. Wetlands 22:616-625.

Fogli, S., R. Marchesini, and R. Gerdol. 2002. Reed (Phragmites australis) decline in a brackish wetland in Italy. Marine Environmental Research 53:465-479.

Freeman, A., and S. L. Durden. 1998. A three-component scattering model for polarimetric SAR data. IEEE Transactions on Geoscience and Remote Sensing 36:963-973.

Gagnon, Z. E., and J. M. Glime. 1992. The pH-lowering ability of Sphagnum magellanicum Brid. Journal of bryology 17:47-57.

Georgiev, T., Z. Yu, A. Lumsdaine, and S. Goma. 2013. Lytro camera technology: theory, algorithms, performance analysis. Page $86671 \mathrm{~J}$ Proc. SPIE.

Gitelson, A. A., and M. N. Merzlyak. 1996. Signature analysis of leaf reflectance spectra: algorithm development for remote sensing of chlorophyll. Journal of plant physiology 148:494-500.

Goldberg, D. E. 1990. Components of resource competition in plant communities. Perspectives on plant competition:27-49.

Gorthi, S. S., and E. Schonbrun. 2012. Phase imaging flow cytometry using a focus-stack collecting microscope. Optics letters 37:707-709.

Van der Graaf, A. J., D. Bos, M. J. J. E. Loonen, M. Engelmoer, and R. H. Drent. 2002. Short-term and long-term facilitation of goose grazing by livestock in the Dutch Wadden Sea area. Journal of Coastal Conservation 8:179-188.

Graham, a. J., and R. Harris. 2003. Extracting biophysical parameters from remotely sensed radar data: a review of the water cloud model. Progress in Physical Geography 27:217-229.

Grime, J. P. 1974. Vegetation classification by reference to strategies. Nature 250:26-31. 
Grime, J. P. 1977. Evidence for the existence of three primary strategies in plants and its relevance to ecological and evolutionary theory. American naturalist:1169-1194.

de Groot, A. V, R. M. Veeneklaas, D. P. J. Kuijper, and J. P. Bakker. 2011. Spatial patterns in accretion on barrier-island salt marshes. Geomorphology 134:280-296.

Grossmann, P. 1987. Depth from focus. Pattern recognition letters 5:63-69.

Guerini Filho, M., T. M. Kuplich, and F. L. F. De Quadros. 2020. Estimating natural grassland biomass by vegetation indices using Sentinel 2 remote sensing data. International Journal of Remote Sensing 41:2861-2876.

Gunnarsson, B. 1990. Vegetation structure and the abundance and size distribution of spruce-living spiders. The Journal of Animal Ecology:743-752.

Guo, L., D. W. Ott, and T. J. Cutright. 2014. Accumulation and histological location of heavy metals in Phragmites australis grown in acid mine drainage contaminated soil with or without citric acid. Environmental and Experimental Botany 105:46-54.

Guthery, F. S., T. B. Doerr, and M. A. Taylor. 1981. Use of a profile board in sand shinnery oak communities. Rangeland Ecology \& Management/Journal of Range Management Archives 34:157-158.

El Hajj, M., N. Baghdadi, H. Bazzi, and M. Zribi. 2019. Penetration analysis of SAR signals in the C and $L$ bands for wheat, maize, and grasslands. Remote Sensing 11:31.

Hallmann, C. A., M. Sorg, E. Jongejans, H. Siepel, N. Hofland, H. Schwan, W. Stenmans, A. Müller, H. Sumser, and T. Hörren. 2017. More than 75 percent decline over 27 years in total flying insect biomass in protected areas. PloS one 12:e0185809.

Hanganu, J., D. Dubyna, E. Zhmud, I. Grigoras, U. Menke, H. Drost, N. Ştefan, and I. Sărbu. 2002. Vegetation of the Biosphere Reserve "Danube Delta." Page RIZA report 2002.049.

Hanganu, J., G. Mihail, and H. Coops. 1999. Responses of ecotypes of Phragmites australis to increased seawater influence: a field study in the Danube Delta, Romania. Aquatic Botany 64:351-358.

Harmoney, K. R., K. J. Moore, J. R. George, E. C. Brummer, and J. R. Russell. 1997. Determination of pasture biomass using four indirect methods. Agronomy Journal 89:665672.

Harrell, W. C., and S. D. Fuhlendorf. 2002. Evaluation of habitat structural measures in a shrubland community. Journal of Range Management:488-493.

Harvey, P. 2007. No Title. http://web.mit.edu/graphics/src/lmage-ExifTool-6.99/html/index.html.

Haslam, S. M. 1970. The performance of Phragmites communis Trin. in relation to water-supply. Annals of Botany 34:867-877.

Henderson, F. M., and A. J. Lewis. 2008. Radar detection of wetland ecosystems: a review. International Journal of Remote Sensing 29:5809-5835.

Hickey, S. M., N. J. Callow, S. Phinn, C. E. Lovelock, and C. M. Duarte. 2018. Spatial complexities in aboveground carbon stocks of a semi-arid mangrove community: A remote sensing heightbiomass-carbon approach. Estuarine, Coastal and Shelf Science 200:194-201.

Hill, M. J., G. E. Donald, and P. J. Vickery. 1999. Relating radar backscatter to biophysical properties of temperate perennial grassland. Remote Sensing of Environment 67:15-31.

Hladik, C., and M. Alber. 2012. Accuracy assessment and correction of a LIDAR-derived salt marsh digital elevation model. Remote Sensing of Environment 121:224-235.

Hladik, C., and M. Alber. 2014. Classification of salt marsh vegetation using edaphic and remote sensing-derived variables. Estuarine, Coastal and Shelf Science 141:47-57.

Homolova, L., Z. Malenovský, J. G. P. W. Clevers, G. García-Santos, and M. E. Schaepman. 2013. Review of optical-based remote sensing for plant trait mapping. Ecological Complexity 15:116.

Imhoff, M. L., T. D. Sisk, A. Milne, G. Morgan, and T. Orr. 1997. Remotely sensed indicators of 
habitat heterogeneity: use of synthetic aperture radar in mapping vegetation structure and bird habitat. Remote Sensing of Environment 60:217-227.

Jacobs, D. E., J. Baek, and M. Levoy. 2012. Focal stack compositing for depth of field control. Stanford Computer Graphics Laboratory Technical Report 1:2012.

Jacquemoud, S. 1993. Inversion of the PROSPECT+ SAIL canopy reflectance model from AVIRIS equivalent spectra: theoretical study. Remote sensing of environment 44:281-292.

Jacquemoud, S., and F. Baret. 1990. PROSPECT: A model of leaf optical properties spectra. Remote Sensing of Environment 34:75-91.

Jacquemoud, S., W. Verhoef, F. Baret, C. Bacour, P. J. Zarco-Tejada, G. P. Asner, C. François, and S. L. Ustin. 2009. PROSPECT+ SAIL models: A review of use for vegetation characterization. Remote sensing of environment 113:S56-S66.

Jagdhuber, T., C. Montzka, C. Lopez-Martinez, M. J. Baur, M. Link, M. Piles, N. N. Das, and F. Jonard. 2020. Estimation of Vegetation Structure Parameters From SMAP Radar Intensity Observations. IEEE Transactions on Geoscience and Remote Sensing.

Jensen, J. R., C. Coombs, D. Porter, B. Jones, S. Schill, and D. White. 1998. Extraction of smooth cordgrass (Spartina alterniflora) biomass and leaf area index parameters from high resolution imagery. Geocarto International 13:25-34.

Jerath, M., M. Bhat, V. H. Rivera-Monroy, E. Castañeda-Moya, M. Simard, and R. R. Twilley. 2016. The role of economic, policy, and ecological factors in estimating the value of carbon stocks in Everglades mangrove forests, South Florida, USA. Environmental Science \& Policy 66:160-169.

Joern, A. 1982. Vegetation structure and microhabitat selection in grasshoppers (Orthoptera, Acrididae). The Southwestern Naturalist:197-209.

Jones, C. G., J. L. Gutiérrez, J. E. Byers, J. A. Crooks, J. G. Lambrinos, and T. S. Talley. 2010. A framework for understanding physical ecosystem engineering by organisms. Oikos 119:1862-1869.

Kazmi, W., S. Foix, G. Alenya, and H. Jørgen. 2014. Indoor and Outdoor Depth Imaging of Leaves With Time of Flight and stereo vision Sensors : Analysis and Comparison. ISPRS Journal of Photogrammetry and Remote Sensing.

Kearney, M. S., D. Stutzer, K. Turpie, and J. C. Stevenson. 2009. The effects of tidal inundation on the reflectance characteristics of coastal marsh vegetation. Journal of Coastal Research 25:1177-1186.

Kéfi, S., V. Dakos, M. Scheffer, E. H. Van Nes, and M. Rietkerk. 2013. Early warning signals also precede non-catastrophic transitions. Oikos 122:641-648.

Kerr, J. T., and M. Ostrovsky. 2003. From space to species: ecological applications for remote sensing. Trends in Ecology \& Evolution 18:299-305.

Klemas, V. 2013. Remote sensing of coastal wetland biomass: An overview. Journal of Coastal Research 29:1016-1028.

Koch, E. W., E. B. Barbier, B. R. Silliman, D. J. Reed, G. M. E. Perillo, S. D. Hacker, E. F. Granek, J. H. Primavera, N. Muthiga, S. Polasky, B. S. Halpern, C. J. Kennedy, C. V Kappel, and E. Wolanski. 2009. Non-linearity in ecosystem services: temporal and spatial variability in coastal protection. Frontiers in Ecology and the Environment 7:29-37.

Kooistra, L., and J. G. P. W. Clevers. 2016. Estimating potato leaf chlorophyll content using ratio vegetation indices. Remote Sensing Letters 7:611-620.

Kotchenova, S. Y., and E. F. Vermote. 2007. Validation of a vector version of the 6 S radiative transfer code for atmospheric correction of satellite data Part II Homogeneous Lambertian and anisotropic surfaces. Applied Optics 46:4455.

Kromkamp, J. C., E. P. Morris, R. M. Forster, C. Honeywill, S. Hagerthey, and D. M. Paterson. 2006. Relationship of intertidal surface sediment chlorophyll concentration to hyperspectral 
reflectance and chlorophyll fluorescence. Estuaries and Coasts 29:183-196.

Kruse, F. A., A. B. Lefkoff, J. W. Boardman, K. B. Heidebrecht, A. T. Shapiro, P. J. Barloon, and A. F. H. Goetz. 1993. The spectral image processing system (SIPS)—interactive visualization and analysis of imaging spectrometer data. Remote Sensing of Environment 44:145-163.

Lawley, V., M. Lewis, K. Clarke, and B. Ostendorf. 2016. Site-based and remote sensing methods for monitoring indicators of vegetation condition: An Australian review. Ecological Indicators 60:1273-1283.

Lee, Y.-K., J.-W. Park, J.-K. Choi, Y. Oh, and J.-S. Won. 2012. Potential uses of TerraSAR-X for mapping herbaceous halophytes over salt marsh and tidal flats. Estuarine, Coastal and Shelf Science 115:366-376.

Legendre, P., and M. J. Fortin. 1989. Spatial pattern and ecological analysis. Vegetatio 80:107138.

Lehnert, L. W. 2016. References for package " hsdar" 39:1-7.

Li, L., Q. Zhang, and D. Huang. 2014. A review of imaging techniques for plant phenotyping. Sensors (Basel, Switzerland) 14:20078-20111.

Li, Z., X. Jin, J. Wang, G. Yang, C. Nie, X. Xu, and H. Feng. 2015. Estimating winter wheat (Triticum aestivum) LAI and leaf chlorophyll content from canopy reflectance data by integrating agronomic prior knowledge with the PROSAIL model. International journal of remote sensing 36:2634-2653.

Lichtenthaler, H. K. 1996. Vegetation stress: an introduction to the stress concept in plants. Journal of plant physiology 148:4-14.

Lichtenthaler, H. K., M. Lang, M. Sowinska, F. Heisel, and J. A. Miehe. 1996. Detection of vegetation stress via a new high resolution fluorescence imaging system. Journal of plant physiology 148:599-612.

Lindau, C. W., R. D. DeLaune, A. Jugsujinda, and E. Sajo. 1999. Response of Spartina alterniflora vegetation to oiling and burning of applied oil. Marine Pollution Bulletin 38:1216-1220.

Lobell, D. B., S. M. Lesch, D. L. Corwin, M. G. Ulmer, K. A. Anderson, D. J. Potts, J. A. Doolittle, M. R. Matos, and M. J. Baltes. 2010. Regional-scale assessment of soil salinity in the Red River Valley using multi-year MODIS EVI and NDVI. Journal of environmental quality 39:35-41.

Lou, Y., S. Hensley, B. Hawkins, C. Jones, M. Lavalle, T. Michel, D. Moller, R. Muellerschoen, N. Pinto, and X. Wu. 2017. Uavsar program: Recent upgrades to support vegetation structure studies and land ICE topography mapping. Pages 5893-58952017 IEEE International Geoscience and Remote Sensing Symposium (IGARSS). IEEE.

Luckman, A., J. Baker, M. Honzák, and R. Lucas. 1998. Tropical forest biomass density estimation using JERS-1 SAR: Seasonal variation, confidence limits, and application to image mosaics. Remote Sensing of Environment 63:126-139.

Van der Maarel, E. 1971. Florastatistieken als bijdrage tot de evaluatie van natuurgebieden. Gorteria 5:176-188.

Maas, H., A. Bienert, S. Scheller, and E. Keane. 2008. Automatic forest inventory parameter determination from terrestrial laser scanner data. International journal of remote sensing 29:1579-1593.

Maes, J., B. Egoh, L. Willemen, C. Liquete, P. Vihervaara, J. P. Schägner, B. Grizzetti, E. G. Drakou, A. La Notte, and G. Zulian. 2012. Mapping ecosystem services for policy support and decision making in the European Union. Ecosystem services 1:31-39.

Maes, J., C. Liquete, A. Teller, M. Erhard, M. L. Paracchini, J. I. Barredo, B. Grizzetti, A. Cardoso, F. Somma, and J.-E. Petersen. 2016. An indicator framework for assessing ecosystem services in support of the EU Biodiversity Strategy to 2020. Ecosystem services 17:14-23.

Marani, M., C. Da Lio, and A. D'Alpaos. 2013. Vegetation engineers marsh morphology through multiple competing stable states. Proceedings of the National Academy of Sciences 
110:3259-3263.

Martín, J., and P. López. 1998. Shifts in microhabitat use by the lizard Psammodromus algirus: responses to seasonal changes in vegetation structure. Copeia 1998:780-786.

Mateos-Naranjo, E., S. Redondo-Gómez, J. Cambrollé, and M. E. Figueroa. 2008. Growth and photosynthetic responses to copper stress of an invasive cordgrass, Spartina densiflora. Marine Environmental Research 66:459-465.

McClure, A., X. Liu, E. Hines, and M. C. Ferner. 2016. Evaluation of error reduction techniques on a lidar-derived salt marsh digital elevation model. Journal of Coastal Research 32:424-433.

Meijden, R. van der. (n.d.). Heukels' flora van Nederland. 22e druk. Wolter-Noordhof, Groningen.

Millennium Ecosystem Assessment. 2005. Ecosystems and human well-being. Island Press Washington, DC.

Mobasheri, M. R., and S. B. Fatemi. 2013. Leaf Equivalent Water Thickness assessment using reflectance at optimum wavelengths. Theoretical and Experimental Plant Physiology 25:196202.

Möckel, T., O. Löfgren, H. C. Prentice, L. Eklundh, and K. Hall. 2016. Airborne hyperspectral data predict Ellenberg indicator values for nutrient and moisture availability in dry grazed grasslands within a local agricultural landscape. Ecological Indicators 66:503-516.

Moffett, K. B., D. A. Robinson, and S. M. Gorelick. 2010. Relationship of salt marsh vegetation zonation to spatial patterns in soil moisture, salinity, and topography. Ecosystems 13:12871302.

Möller, I. 2006. Quantifying saltmarsh vegetation and its effect on wave height dissipation: Results from a UK East coast saltmarsh. Estuarine, Coastal and Shelf Science 69:337-351.

Möller, I., M. Kudella, F. Rupprecht, T. Spencer, M. Paul, B. K. Van Wesenbeeck, G. Wolters, K. Jensen, T. J. Bouma, and M. Miranda-Lange. 2014. Wave attenuation over coastal salt marshes under storm surge conditions. Nature Geoscience 7:727-731.

Moran, P. A. P. 1950. Notes on continuous stochastic phenomena. Biometrika 37:17-23.

Morgan, P. A., D. M. Burdick, and F. T. Short. 2009. The functions and values of fringing salt marshes in northern New England, USA. Estuaries and Coasts 32:483-495.

Morris, J. T. 1989. Modelling light distribution within the canopy of the marsh grass Spartina alterniflora as a function of canopy biomass and solar angle. Agricultural and Forest Meteorology 46:349-361.

Mulla, D. J. 2013. Twenty five years of remote sensing in precision agriculture: Key advances and remaining knowledge gaps. Biosystems engineering 114:358-371.

Murad, H., and A. Islam. 2011. Drought assessment using remote sensing and GIS in north-west region of Bangladesh. Pages 797-804Proceedings of the 3rd International Conference on Water \& Flood Management.

Mutanga, O., and A. K. Skidmore. 2004. Narrow band vegetation indices overcome the saturation problem in biomass estimation. International Journal of Remote Sensing 25:3999-4014.

van Nes, E. H., and M. Scheffer. 2007. Slow Recovery from Perturbations as a Generic Indicator of a Nearby Catastrophic Shift. The American Naturalist 169:738-747.

Neumeier, U., and P. Ciavola. 2004. Flow resistance and associated sedimentary processes in a Spartina maritima salt-marsh. Journal of Coastal Research:435-447.

Nock, C. A., O. Taugourdeau, S. Delagrange, and C. Messier. 2013. Assessing the potential of lowcost 3D cameras for the rapid measurement of plant woody structure. Sensors (Basel, Switzerland) 13:16216-16233.

Obermaier, E., A. Heisswolf, J. Poethke, B. Randlkofer, and T. Meiners. 2008. Plant architecture and vegetation structure: Two ways for insect herbivores to escape parasitism. European Journal of Entomology 105:233-240.

Ostendorp, W., M. Dienst, and K. Schmieder. 2003. Disturbance and rehabilitation of lakeside 
Phragmites reeds following an extreme flood in Lake Constance (Germany). Hydrobiologia 506-509:687-695.

Oteman, B., E. P. Morris, G. Peralta, T. J. Bouma, and D. van der Wal. 2019. Using Remote Sensing to Identify Drivers behind Spatial Patterns in the Bio-physical Properties of a Saltmarsh Pioneer. Remote sensing 11:511.

Ouyang, Z.-T., Y. Gao, X. Xie, H.-Q. Guo, T.-T. Zhang, and B. Zhao. 2013. Spectral discrimination of the invasive plant Spartina alterniflora at multiple phenological stages in a saltmarsh wetland. PloS one 8:e67315.

Paree, E. 2017. Toelichting op de zoute ecotopenkaart Westerschelde 2016 - Biologische monitoring zoute rijkswateren:25 pp.

Patel, S. 2016. Salicornia: evaluating the halophytic extremophile as a food and a pharmaceutical candidate. 3 Biotech 6:104.

Pengra, B. W., C. A. Johnston, and T. R. Loveland. 2007. Mapping an invasive plant, Phragmites australis, in coastal wetlands using the EO-1 Hyperion hyperspectral sensor. Remote Sensing of Environment 108:74-81.

Pennings, S. C., and R. M. Callaway. 1992. Salt marsh plant zonation: the relative importance of competition and physical factors. Ecology 73:681-690.

Pethick, J. 2001. Coastal management and sea-level rise. CATENA 42:307-322.

Pettengill, T. M., S. M. Crotty, C. Angelini, and M. D. Bertness. 2018. A natural history model of New England salt marsh die-off. Oecologia 186:621-632.

Pettorelli, N., J. O. Vik, A. Mysterud, J.-M. Gaillard, C. J. Tucker, and N. C. Stenseth. 2005. Using the satellite-derived NDVI to assess ecological responses to environmental change. Trends in ecology \& evolution 20:503-510.

Pimm, S. L. 1984. The complexity and stability of ecosystems. Nature 307:321-326.

Poulin, B., A. Davranche, and G. Lefebvre. 2010. Ecological assessment of Phragmites australis wetlands using multi-season SPOT-5 scenes. Remote Sensing of Environment 114:16021609.

Proisy, C., E. Mougin, E. Dufrêne, and V. Le Dantec. 2000. Monitoring seasonal changes of a mixed temperate forest using ERS SAR observations. IEEE Transactions on Geoscience and Remote Sensing 38:540-552.

Van Der Putten, W. H. 1997. Die-back of Phragmites australis in European wetlands: An overview of the European Research Programme on Reed Die-back and Progression (1993-1994). Aquatic Botany 59:263-275.

Reif, M. K., and H. J. Theel. 2017. Remote sensing for restoration ecology: Application for restoring degraded, damaged, transformed, or destroyed ecosystems. Integrated environmental assessment and management 13:614-630.

Reschke, J., A. Bartsch, S. Schlaffer, and D. Schepaschenko. 2012. Capability of C-band SAR for operational wetland monitoring at high latitudes. Remote Sensing 4:2923-2943.

Rhee, M. H., H.-J. Park, and J. Y. Cho. 2009. Salicornia herbacea: Botanical, chemical and pharmacological review of halophyte marsh plant. Journal of Medicinal Plants Research 3:548-555.

Rizal, A., A. Sahidin, and H. Herawati. 2018. Economic Value Estimation of Mangrove Ecosystems in Indonesia. Biodiversity Int J 2:51.

Roberts, D. A., K. L. Roth, and R. L. Perroy. 2016. Hyperspectral Vegetation Indices. Pages 344363Hyperspectral Remote Sensing of Vegetation. CRC Press.

Rogers, J. N., C. E. Parrish, L. G. Ward, and D. M. Burdick. 2018. Improving salt marsh digital elevation model accuracy with full-waveform lidar and nonparametric predictive modeling. Estuarine, Coastal and Shelf Science 202:193-211.

Rönnbäck, P. 1999. The ecological basis for economic value of seafood production supported by 
mangrove ecosystems. Ecological Economics 29:235-252.

Rupprecht, F., I. Möller, B. Evans, T. Spencer, and K. Jensen. 2015. Biophysical properties of salt marsh canopies-Quantifying plant stem flexibility and above ground biomass. Coastal Engineering 100:48-57.

Samanta, A., S. Ganguly, H. Hashimoto, S. Devadiga, E. Vermote, Y. Knyazikhin, R. R. Nemani, and R. B. Myneni. 2010. Amazon forests did not green-up during the 2005 drought. Geophysical research letters 37.

Sanderson, E. W., T. C. Foin, and S. L. Ustin. 2001. A simple empirical model of salt marsh plant spatial distributions with respect to a tidal channel network. Ecological Modelling 139:293307.

Scheffer, M., J. Bascompte, W. A. Brock, V. Brovkin, S. R. Carpenter, V. Dakos, H. Held, E. H. Van Nes, M. Rietkerk, and G. Sugihara. 2009. Early-warning signals for critical transitions. Nature 461:53-59.

Scheffer, M., S. Carpenter, J. A. Foley, C. Folke, and B. Walker. 2001. Catastrophic shifts in ecosystems. Nature 413:591-596.

Schima, R., H. Mollenhauer, G. Grenzdörffer, I. Merbach, P. Dietrich, and J. Bumberger. 2016. Imagine All the Plants : Evaluation of a Light-Field Camera for On-Site Crop Growth Monitoring. Remote Sensing 8:823.

Schmidt, J., F. E. Fassnacht, M. Förster, and S. Schmidtlein. 2018. Synergetic use of Sentinel-1 and Sentinel-2 for assessments of heathland conservation status. Remote Sensing in Ecology and Conservation 4:225-239.

Schmidt, J., F. E. Fassnacht, C. Neff, A. Lausch, B. Kleinschmit, M. Förster, and S. Schmidtlein. 2017. Adapting a Natura 2000 field guideline for a remote sensing-based assessment of heathland conservation status. International journal of applied earth observation and geoinformation 60:61-71.

Schmidt, K. S., and A. K. Skidmore. 2003. Spectral discrimination of vegetation types in a coastal wetland. Remote sensing of Environment 85:92-108.

Schmidtlein, S. 2005. Imaging spectroscopy as a tool for mapping Ellenberg indicator values. Journal of Applied ecology 42:966-974.

Shugart, H. H., S. Saatchi, and F. G. Hall. 2010. Importance of structure and its measurement in quantifying function of forest ecosystems. Journal of Geophysical Research: Biogeosciences 115.

Si, Y., M. Schlerf, R. Zurita-Milla, A. Skidmore, and T. Wang. 2012. Mapping spatio-temporal variation of grassland quantity and quality using MERIS data and the PROSAIL model. Remote Sensing of Environment 121:415-425.

Silliman, B. R., and M. D. Bertness. 2004. Shoreline Development Drives Invasion of Phragmites australis and the Loss of Plant Diversity on New England Salt Marshes. Conservation Biology 18:1424-1434.

Silvestri, S., A. Defina, and M. Marani. 2005. Tidal regime, salinity and salt marsh plant zonation. Estuarine, coastal and shelf science 62:119-130.

Silvestri, S., and M. Marani. 2004. Salt-Marsh Vegetation and Morphology: Basic Physiology, Modelling and Remote Sensing Observations. The Ecogeomorphology of Tidal Marshes:525.

Silvestri, S., M. Marani, and A. Marani. 2003. Hyperspectral remote sensing of salt marsh vegetation, morphology and soil topography. Physics and Chemistry of the Earth, Parts a/B/C 28:15-25.

Soissons, L. M., Q. Han, B. Li, M. M. van Katwijk, T. Ysebaert, P. M. J. Herman, and T. J. Bouma. 2014. Cover versus recovery: contrasting responses of two indicators in seagrass beds. Marine pollution bulletin 87:211-219. 
Soissons, L. M., B. Li, Q. Han, M. M. van Katwijk, T. Ysebaert, P. M. J. Herman, and T. J. Bouma. 2016. Understanding seagrass resilience in temperate systems: the importance of timing of the disturbance. Ecological indicators 66:190-198.

Sozzi, M., F. Marinello, A. Pezzuolo, and L. Sartori. (n.d.). Benchmark of Satellites Image Services for Precision Agricultural use. AgEng Conference Wageningen.

Specht, R. L., E. J. Moll, F. Pressinger, and J. Sommerville. 1983. Moisture regime and nutrient control of seasonal growth in Mediterranean ecosystems. Pages 120-132Mediterranean-type ecosystems. Springer.

Stanica, A., J. Bujini, and D. Stefanescu. 2012. Architecture and roadmap to manage multiple pressures on lagoons.

Sun, X., Y. Xu, Q. Zhang, X. Li, and Z. Yan. 2018. Combined effect of water inundation and heavy metals on the photosynthesis and physiology of Spartina alterniflora. Ecotoxicology and environmental safety 153:248-258.

Temmerman, S., P. Meire, T. J. Bouma, P. M. J. Herman, T. Ysebaert, and H. J. De Vriend. 2013. Ecosystem-based coastal defence in the face of global change. Nature 504:79.

Tolman, M. E., and D. P. Pranger. 2012. Toelichting bij de Vegetatiekartering Westerschelde 2010. https://geoservices.rijkswaterstaat.nl/apps/geonetwor:1-114.

Tong, A., and Y. He. 2017. Estimating and mapping chlorophyll content for a heterogeneous grassland: Comparing prediction power of a suite of vegetation indices across scales between years. ISPRS Journal of Photogrammetry and Remote Sensing 126:146-167.

Tripathi, R., R. N. Sahoo, V. K. Sehgal, V. K. Gupta, B. B. K. Bhattacharya, .K. Gupta, and B. B. K. Bhattacharya. 2009. Remote Sensing Derived Composite Vegetation Health Index Through Inversion of Prosail for Monitoring of Wheat Growth in Trans Gangetic Plains of India. ISPRS Archives XXXVIII-8/W3 Workshop Proceedings: Impact of Climate Change on Agriculture:319-325.

Tripathi, R., R. N. Sahoo, V. K. Sehgal, R. K. Tomar, D. Chakraborty, and S. Nagarajan. 2012. Inversion of PROSAIL model for retrieval of plant biophysical parameters. Journal of the Indian Society of Remote Sensing 40:19-28.

Tyc, G., J. Tulip, D. Schulten, M. Krischke, and M. Oxfort. 2005. The RapidEye mission design. Acta Astronautica 56:213-219.

Uossef Gomrokchi, A., M. Akbari, A. Hassanoghli, and M. Younesi. 2020. Monitoring Soil Salinity and Vegetation Using Multispectral Remote Sensing Data in Interceptor Drain of Salt Marsh in Qazvin Plain.

Valladares, F., and Ü. Niinemets. 2007. The architecture of plant crowns. Page Functional Plant Ecology, Second Edition. CRC Press.

Veraart, A. J., E. J. Faassen, V. Dakos, E. H. van Nes, M. Lürling, and M. Scheffer. 2012. Recovery rates reflect distance to a tipping point in a living system. Nature 481:357-359.

Verger, A., F. Baret, and F. Camacho. 2011. Optimal modalities for radiative transfer-neural network estimation of canopy biophysical characteristics: Evaluation over an agricultural area with CHRIS/PROBA observations. Remote Sensing of Environment 115:415-426.

Verhoef, W. 1984. Light scattering by leaf layers with application to canopy reflectance modeling: the SAIL model. Remote sensing of environment 16:125-141.

Vilibić, I., and J. Šepić. 2010. Long-term variability and trends of sea level storminess and extremes in European Seas. Global and planetary change 71:1-12.

Vincini, M., E. Frazzi, and P. D'Alessio. 2008. A broad-band leaf chlorophyll vegetation index at the canopy scale. Precision Agriculture 9:303-319.

Visser, J. M., C. E. Sasser, and B. S. Cade. 2006. The effect of multiple stressors on salt marsh end-of-season biomass. Estuaries and Coasts 29:328-339.

Volesky, L. A., S. Iqbal, J. J. Kelly, and P. Geddes. 2018. Relationships of Native and Exotic Strains 
of Phragmites australis to Wetland Ecosystem Properties. Wetlands 38:577-589.

Van Der Wal, D., and P. M. J. Herman. 2007. Regression-based synergy of optical, shortwave infrared and microwave remote sensing for monitoring the grain-size of intertidal sediments. Remote Sensing of Environment 111:89-106.

Van der Wal, D., P. M. J. Herman, R. M. Forster, T. Ysebaert, F. Rossi, E. Knaeps, Y. M. G. Plancke, and S. J. Ides. 2008a. Distribution and dynamics of intertidal macrobenthos predicted from remote sensing: response to microphytobenthos and environment. Marine Ecology Progress Series 367:57-72.

Van der Wal, D., A. Wielemaker-Van den Dool, and P. M. J. Herman. 2008b. Spatial patterns, rates and mechanisms of saltmarsh cycles (Westerschelde, The Netherlands). Estuarine, Coastal and Shelf Science 76:357-368.

Wallis de Vries, M. F., and C. A. M. Van Swaay. 2006. Global warming and excess nitrogen may induce butterfly decline by microclimatic cooling. Global Change Biology 12:1620-1626.

Wamelink, G. W. W., P. W. Goedhart, H. F. Van Dobben, and F. Berendse. 2005. Plant species as predictors of soil $\mathrm{pH}$ : replacing expert judgement with measurements. Journal of vegetation science 16:461-470.

Wang, H., D. Wal, X. Li, J. Belzen, P. M. J. Herman, Z. Hu, Z. Ge, L. Zhang, and T. J. Bouma. 2017. Zooming in and out: scale-dependence of extrinsic and intrinsic factors affecting salt marsh erosion. Journal of Geophysical Research: Earth Surface.

Wang, R., and J. A. Gamon. 2019. Remote sensing of terrestrial plant biodiversity. Remote Sensing of Environment 231:111218.

Weisner, S. E. B. 1987. The relation between wave exposure and distribution of emergent vegetation in a eutrophic lake. Freshwater Biology 18:537-544.

Westhoff, V. 1967. Problems and use of structure in the classification of vegetation. The diagnostic evaluation of structure in the Braun-Blanquet system. Acta botanica neerlandica 15:495-511.

Westoby, M. J., J. Brasington, N. F. Glasser, M. J. Hambrey, and J. M. Reynolds. 2012. "Structurefrom-Motion" photogrammetry: A low-cost, effective tool for geoscience applications. Geomorphology 179:300-314.

Xin, P., L. Li, and D. A. Barry. 2013. Tidal influence on soil conditions in an intertidal creek-marsh system. Water Resources Research.

Yamasaki, S., and I. Tange. 1981. Growth responses of Zizania latifolia, Phragmites australis and Miscanthus sacchariflorus to varying inundation. Aquatic Botany 10:229-239.

Zarco-Tejada, P. J., J. R. Miller, G. H. Mohammed, T. L. Noland, and P. H. Sampson. 2002. Vegetation stress detection through chlorophyll $a+b$ estimation and fluorescence effects on hyperspectral imagery. Journal of environmental quality 31:1433-1441.

Zedler, J. B., J. C. Callaway, J. S. Desmond, G. Vivian-Smith, G. D. Williams, G. Sullivan, A. E. Brewster, and B. K. Bradshaw. 1999. Californian salt-marsh vegetation: an improved model of spatial pattern. Ecosystems 2:19-35.

Zhang, C., D. R. Mishra, and S. C. Pennings. 2019. Mapping salt marsh soil properties using imaging spectroscopy. ISPRS Journal of Photogrammetry and Remote Sensing 148:221234.

Zhang, Q., C. Schaaf, and K. C. Seto. 2013. The vegetation adjusted NTL urban index: A new approach to reduce saturation and increase variation in nighttime luminosity. Remote Sensing of Environment 129:32-41.

Zhao, Q., J. Bai, Q. Liu, Q. Lu, Z. Gao, and J. Wang. 2016. Spatial and Seasonal Variations of Soil Carbon and Nitrogen Content and Stock in a Tidal Salt Marsh with Tamarix chinensis, China. Wetlands 36:145-152.

Zheng, G., and L. M. Moskal. 2009. Retrieving leaf area index (LAI) using remote sensing: theories, methods and sensors. Sensors 9:2719-2745. 
Zheng, Z., Y. Zhou, B. Tian, and X. Ding. 2016. The spatial relationship between salt marsh vegetation patterns, soil elevation and tidal channels using remote sensing at Chongming Dongtan Nature Reserve, China. Acta Oceanologica Sinica 35:26-34.

Zhou, Z., Y. Yang, and B. Chen. 2018. Estimating Spartina alterniflora fractional vegetation cover and aboveground biomass in a coastal wetland using SPOT6 satellite and UAV data. Aquatic Botany 144:38-45.

Zhu, Z., Z. Yang, and T. J. Bouma. 2020. Biomechanical properties of marsh vegetation in space and time: effects of salinity, inundation and seasonality. Annals of Botany 125:277-290. 


\section{Summary}

Humanity uses many services provided by ecosystems; the stability of these services should therefore be monitored carefully. Coastal ecosystems provide many such services, for example: wave mitigation and flood protection, increased tourism, and the provision of food directly (e.g., fish) or by providing food for livestock. In this thesis we look at how coastal marshes can be assessed and monitored effectively, to be informed on the stability of the services they provide. It is important to realize that, in a natural ecosystem, many species are interdependent, and contribute to the stability of a service. A shift in vegetation composition could therefore affect the stability of ecosystem service provision, these shifts are generally preceded by an increase in vegetation stress. High stress in vegetation can also directly affect service provision. In this thesis, we focus on the pioneer zone of coastal marsh vegetation. The main question in this thesis is: 'how can we establish efficient stress indicators to monitor ecosystem service stability in European coastal marshes, to help safeguard vital ecosystem services'.

As coastal marshes are often difficult to access, we expect radar and optical remote sensing can be an efficient way to provide indicators for vegetation stress. In active radar systems, the backscatter from a microwave pulse is used to determine properties of an object; radar backscatter is, among other variables, depending on the structure of the object. In passive optical remote sensing, sun light that has been reflected of an object is recorded; the reflectance in the visible and near-infrared depends on the objects properties, such as the pigments and biomass of vegetation. Both types of vegetation properties can be expected to be affected by stress, therefore both radar and optical data offer potentially useful data sources.

To gain a better understanding of the underlying processes, we examine stress at three different levels (leaf, plant and community). The three levels can all be measured in situ; space borne platforms can only produce community level data. Our measurements are along an elevation gradient, where we expect, based on literature and validated by our in situ vegetation biomass measurements, high stress due to inundation at low elevations, low stress at intermediate elevations, and high stress at high elevations due to competition.

\section{Vegetation structure (roughness) as stress indicator}

The technique to measure reflectance in situ is well established, but in situ structure measurements remain difficult. Therefore, we developed a new technique, named depth from focus, to allow detailed in situ structure 
measurements of vegetation. This technique consists of a normal DSLR camera taking multiple images from the same location, each with a slightly different focus distance. By calculating in which image each pixel is sharpest, a $3 d$ representation of an object is created. This allows for the creation of a highly detailed representation of the structure of vegetation. This technique was rigorously validated, both in the lab and in field situations, and was found to represent vegetation structure well. The importance of small scale vegetation structure is widely recognized for many aspects of ecology. This technique could therefore make a valuable contribution to many areas of study.

We looked at vegetation structure as stress indicator using our new depth from focus method, and using SAR satellite data. The first showed that the four spatial statistics we applied (i.e., RMSz, Spatial autocorrelation, Tortuosity and Moran'sI) to translate the $3 d$ image into a stress indicator, did not provide a stress indicator that was consistent between multiple species. Although the four tested spatial statistics are often used and well established, they might not be the most appropriate as indicators for stress. Further study into which statistics are most suitable to derive vegetation stress from $3 \mathrm{~d}$ images is recommended.

The application of structure analyses in the Razelm Sinoe Lagoon System, part of the Danube Delta in Romania, showed that vegetation structure can be an important predictor of vegetation development. We used optical satellite images from Landsat-5/8 to establish the development (expansion, stable or lateral loss) of reeds (Phragmites australis) over a 22 year period. Seasonal (spring, summer, winter) variability in the vegetation indices NDVI and SAVI from optical (Sentinel2) satellite data, and backscatter indices from dual-polarized $C$ band $S A R$ (Sentinel-1) satellite data were compared between the different development trends. We found no difference in optical reflectance indices between reed development stages, but the radar data had the largest seasonal differences in backscatter signal in locations where reed was expanding and smallest seasonal differences in areas where reed was decreasing. Overall, this showed that the stability of reed ecosystems, and their corresponding services, can be monitored by quantifying seasonal changes in backscatter of reed lands using radar satellites. This principle looks promising for monitoring other ecosystems as well. Although it should be noted that the radar wavelength has a large influence, in other systems another wavelength might be preferable.

\section{Physical stress indicators and optical stress indicators}

Several potential stress indicators were measured in situ, and we tested if they correlate with inundation and competition stress. The potential stress indicators we tested were leaf pigments (Chl-a, Chl-b, carotene), leaf level reflectance, 
vegetation cover, height, biomass and recovery after disturbance. In situ measurements showed that pigments related strongest to inundation stress, while cover and biomass related significantly to both inundation and competition stress. The response of vegetation height to stress differed for the two saltmarsh species studied (Spartina versus Scirpus). Recovery rate is discussed in more detail in the section 'resilience as stress indicator'.

To establish how the physical stress responses translate into reflectance we used the radiative transfer model PROSAIL to simulate reflectance in saltmarshes at the leaf and canopy scale. The relative contribution of a specific property to the reflectance gives an indication of the likely signal to noise ratio. PROSAIL includes optical biochemical characteristics at the leaf scale (e.g., pigments) and optical structural properties at the leaf scale, as well as structural properties at the canopy scale (e.g., Leaf Area Index). Simulations showed that the range in pigment levels found in Spartina plants along the inundation gradient in a Dutch saltmarsh had little effect on reflectance. This makes it very unlikely that stress can be modelled through pigments using space borne data in salt marshes. In contrast, the simulations also showed that especially Leaf Area Index (LAI), which is closely related to biomass, had a large range of values in a saltmarsh, and had a large effect on reflectance.

To establish which stressor had the most influence on reflectance, we inverted the PROSAIL model and applied it to a RapidEye satellite image; this allowed us to estimate LAI of every $5 \times 5 \mathrm{~m}$ cell in a Dutch salt marsh. We correlated these LAI estimates with flood duration, wave forcing, proximity to creeks, and competition. This showed that in the pioneering zone of the Dutch salt marsh for which the model was calibrated, especially flood duration had a large influence on LAI development and hence reflectance.

Along the inundation and competition gradient in two Dutch saltmarshes, we measured reflectance in situ at leaf and community level. At leaf level we saw the vegetation index NDVI representing inundation stress reasonably well for two pioneer species (Spartina and Scirpus), in one of the studied species (Spartina) it also represented competition stress. At leaf level, the REIP (red edge inflection point) showed much variation and was not significantly related to flood duration or competition. At the community level, we found the reflectance-based index REIP to perform better than NDVI in both studies species. This indicates that when choosing a vegetation index, the measurement level is crucial; an index that relates well to stress at the leaf level might not relate well to stress when applied at the community level.

When applying optical stress indicators to the reeds in the Danube delta we found that optical vegetation indices related poorly to the expansion and retraction 
pattern of the reeds and the corresponding expected stress in reed plants. In this case, radar data related much better with the development patterns in reeds. This indicates that stress detection is complex and may be both species and system specific. The choice of indicator should therefore depend on how a stressor affects vegetation, and how this in turn affects reflectance and structure.

\section{Resilience as stress indicator}

Plants can use feedbacks to stabilize their environment and reduce their stress levels; however, when these feedbacks fail, a sudden critical system collapse can take place. Because these feedbacks can mask stress, regular monitoring techniques might not detect it adequately. To be able to detect the approach of such a critical collapse, resilience measurements have been proposed. Resilience can be measured by artificially causing a disturbance and measuring the recovery time. We tested these resilience measurements along an elevation gradient and hence assessed the effect of inundation and competition stress on resilience. We found some effect of inundation stress especially on cover recovery, but the results differed between the two tested pioneer species (Spartina and Scirpus). Recovery of vegetation height only correlated with inundation stress for one species (Scirpus). The interpretation of these data is complex, as the recovery rate represents both the regrowth after the disturbance and potential additional stressors during the recovery period. Therefore the measured recovery rate is the sum of biomass recovery (regrowth) and resilience recovery (recovery of a plants ability to resist additional stressors). Overall we conclude that the technique of using resilience measurements to model stress or system stability has to be developed further before it can be widely applied.

\section{Conclusion and recommendations}

In this study we investigated methods to monitor stress in salt marshes to aid management. We found that there is no single vegetation index that is likely to represent stress well in all salt marshes, and that radar-based indices may perform better than reflectance-based vegetation indices in some systems. Therefore, stress monitoring will have to remain a very area specific task, and although remote sensing will be a valuable tool for these analyses, it will have to be applied by experts who understand the caveats of techniques.

We like to plea for an increased scientific usage of vegetation structure data, both in remote sensing and in situ. Further development of these techniques and an increased understanding of these techniques are required before they can be applied to support management. We would argue for similar caution when applying recovery rate to estimate system stability or system stress. 
To salt marsh managers we would therefore recommend:

- Use remote sensing to monitor stress development in salt marshes, but be sure to have a remote sensing expert and ecologist involved to ensure proper usage of vegetation indices and their interpretation.

- Biomass is still a reliable way to establish stress, however due to its laborintensive nature, it should be reserved for monitoring very vulnerable or critically important systems. Note that potential damage to the system should be taken into account

- Aim to monitor the system as a whole, and include shifts in vegetation type. And scientists we would encourage to:

- support managers by helping to interpret remote sensing data;

- further explore how vegetation structure can be incorporated into monitoring schemes as a stress indicator;

- further validate recovery rate as potential tool to estimate stress in salt marshes;

- continue to develop the tools we present here, expand them to include other sections of the salt marsh to allow for broad ecosystem monitoring. 


\section{Samenvatting}

De mensheid is sterk afhankelijk van ecosysteemdiensten; de stabiliteit van deze diensten moet daarom zorgvuldig gemonitord worden. Kustecosystemen leveren veel van dit soort diensten, zoals golfdemping en bescherming tegen overstroming, toename van toerisme en directe voedselvoorziening (bijvoorbeeld vis) of indirecte voedselvoorziening in de vorm van veevoer. In deze thesis onderzoeken we hoe kustecosystemen het effectiefst beoordeeld en gemonitord kunnen worden, zodat we de stabiliteit van de ecosysteemdiensten die ze leveren kunnen volgen. Het is belangrijk om ons te realiseren dat in een natuurlijk ecosysteem soorten veel onderlinge afhankelijkheden hebben, waardoor veel soorten bijdragen aan de ecosysteemdiensten. Een verschuiving in vegetatietype kan daarom al effect hebben op de stabiliteit van deze diensten. Voor een verschuiving in vegetatietype treedt er over het algemeen een toename van vegetatiestress op. Een hoog vegetatiestress niveau kan ook rechtstreeks het leveren an ecosysteemdiensten beinvloeden. In deze scriptie richten we ons op de pionierszone in zoutmoerasvegaties. De hoofdvraag van deze thesis is: 'Hoe kunnen we efficiënte stressindicatoren vaststellen, om de ecosysteemdiensten van Europese kustmoerassen te monitoren en helpen veilig te stellen?'

Omdat kustmoerassen veelal moeilijk toegankelijk zijn, verwachten we dat indicatoren gebaseerd op radar en optische remote sensing een efficiënte databron kunnen zijn om vegetatiestress te detecteren. In een actief radarsysteem wordt de weerkaatsing van een pulse gebruikt om objecteigenschappen vast te stellen. Naast andere objecteigenschappen is de weerkaatsing afhankelijk van de structuur van het object. In een passief optisch systeem wordt gereflecteerd zonlicht gemeten. Deze reflectie van zichtbaar en nabij infrarood licht hangt in het geval van vegetatie af van objecteigenschappen zoals pigmenten of biomassa. Van beide typen vegetatieeigenschappen kan worden verwacht dat ze veranderen wanneer de plant gestrest is, daarom kunnen zowel radar als optische data bronnen mogelijk nuttig zijn.

Om een beter begrip te krijgen van de onderliggende processen onderzoeken we vegetatiestress op drie verschillende niveaus: blad, plant en gemeenschap. Deze drie niveaus kunnen alledrie in het veld gemeten worden, satellietdatabronnen kunnen alleen data op gemeenschaps-niveau leveren. Onze metingen zijn uitgevoerd langs een hoogtegradiënt, waarbij we op basis van literatuur en gevalideerd door veldmetingen verwachten dat er dicht bij het water relatief veel stress door overstroming optreedt. Bij gemiddelde hoogten verwachten we minder stress, bij hoge bodemhoogte verwachten we een toename van stress omdat hier meer competitie optreedt. 


\section{Vegetatiestructuur (ruwheid) als stressindicator}

De techniek om reflectie te meten in het veld is goed ontwikkeld, maar in het veld vegetatiestructuur meten blijft lastig. Daarom hebben we een nieuwe techniek ontwikkeld genaamd 'depth from focus' waarmee we gedetailleerde structuurmetingen van vegetatie kunnen doen in het veld. Voor deze nieuwe techniek wordt een normale spiegelreflexcamera gebruikt om meerdere foto's te nemen van hetzelfde object, steeds met een kleine verschuiving in het focuspunt. Door voor elke pixel te berekenen in welke foto deze het scherpst is wordt een 3dvoorstelling van het object opgebouwd. Op deze manier kan een zeer gedetailleerde opname van vegetatiestructuur worden gemaakt. Deze nieuwe techniek is uitgebreid gevalideerd, zowel in een laboratorium als in een veldsituatie. Alle validaties lieten zien dat deze techniek de vegetatiestructuur goed kan kwantificeren. Het belang van kleinschalige vegetatiestructuur voor allerlei facetten van ecologie wordt algemeen erkend. Deze nieuwe techniek kan daarom een waardevolle bijdrage leven aan veel verschillende onderzoeksvelden.

Vegetatiestructuur is als stressindicator beoordeeld door te kijken naar veldmetingen middels 'depth from focus' en SAR-satellietdata. De veldmetingen lieten zien dat de vier statistische methoden die we op deze data hebben toegepast (RMSz, spatial autocorrelation, Tortuosity and Moran's-I) om de 3d-opnamen om te zetten naar een stressindicator, niet consistent waren tussen verschillende soorten. Hoewel deze vier statistieken veelgebruikt zijn en goed bekend staan, zijn ze mogelijk niet het meest geschikt als stressindicator. Verder onderzoek zal moeten uitwijzen welke statistieken het best ingezet kunnen worden als stressindicator op basis van $3 d$ structuuropnamen.

De toepassing van structuuranalyses in het Razelm Sinoe Lagunesysteem, onderdeel van de Donaudelta in Roemenië, toonde aan dat vegetatiestructuur een belangrijke voorspeller van vegetatieontwikkeling kan zijn. Op basis van optische satellietbeelden van Landsat-5/8 is de ontwikkeling (uitbreiding, stabiel of lateraal verlies) van riet (Phragmites australis) over een periode van 22 jaar vastgesteld. Seizoensgebonden (lente, zomer, winter) variabiliteit in de vegetatieindices NDVI en SAVI van optische satellietgegevens (Sentinel-2) en radarweerkaatsing van dubbel gepolariseerde C band SAR (Sentinel-1) satellietgegevens is vergeleken voor verschillende ontwikkelingstrends. Dit liet geen verschil zien in optische reflectie-indices tussen de rietuitbreidingsstadia, maar de radardata lieten grote seizoensverschillen zien in het weerkaatsingssignaal op locaties waar riet zich uitbreidde en de kleinste seizoensverschillen in gebieden waar riet afnam. Dit laat zien dat de stabiliteit van rietecosystemen en hun bijbehorende ecosysteemdiensten kan worden gevolgd door het kwantificeren van seizoensveranderingen in de radarweerkaatsing van rietlanden met behulp van 
radarsatellieten. Dit principe ziet er veelbelovend uit, en kan naar verwachting ook worden gebruikt voor het monitoren van andere ecosystemen. Het is belangrijk om op te merken dat de radargolflengte een grote invloed heeft, in een ander systeem kan een andere golflengte de voorkeur hebben.

\section{Fysieke stressindicatoren en optische stressindicatoren}

Verschillende potentiële stressindicatoren zijn in het veld gemeten en er is getest of deze correleren met overstroming- of concurrentiestress. De potentiële stressindicatoren die we hebben getest zijn bladpigmenten (Chl-a, Chl-b, caroteen), reflectie op bladniveau, vegetatiebedekking, hoogte, biomassa en herstel na verstoring. De veldmetingen toonden aan dat pigmenten het sterkst gerelateerd waren aan inundatiestress, terwijl bedekking en biomassa significant gerelateerd waren aan zowel inundatie- als concurrentiestress. De reactie van de vegetatiehoogte op stress verschilde voor de twee onderzochte zoutmoerassoorten (Spartina versus Scirpus). Het herstelpercentage wordt in meer detail besproken in de sectie 'veerkracht als stressindicator'. Om vast te stellen hoe de fysieke stressreacties zich vertalen in reflectantie, gebruikten we het radiative transfer model PROSAIL om reflectantie in zoutmoerassen op blad- en gemeenschapsniveau te simuleren. De relatieve bijdrage van een specifieke eigenschap aan de reflectie geeft een indicatie van de waarschijnlijke signaal-ruisverhouding. PROSAIL omvat optische biochemische kenmerken op de bladschaal (bijv. pigmenten) en optische structurele eigenschappen op de bladschaal, evenals structurele eigenschappen op gemeenschapsniveau (bijvoorbeeld Leaf Area Index). Simulaties lieten zien dat het bereik van pigmentniveaus in Spartina-planten langs de inundatiegradiënt in een Nederlands zoutmoeras weinig effect had op de reflectie. Dit maakt het zeer onwaarschijnlijk dat stress middels pigmenten kan worden gemodelleerd met behulp van satellietdata in zoutmoerassen. Daarentegen toonden de simulaties ook aan dat vooral Leaf Area Index (LAl), een index die nauw verwant is aan biomassa, een groot effect had op de reflectie. Om vast te stellen welke stressor de meeste invloed heeft op de reflectie, is het PROSAIL-model geïnverteerd en toegepast op een RapidEye-satellietbeeld. Hiermee konden we een LAl schatting maken van elke 5x5m cel in een Nederlands zoutmoeras. We correleerden deze LAl-schattingen met overstromingsduur, golfslag, nabijheid van kreken en concurrentie. Hieruit bleek dat in de pionierszone van de Nederlandse kwelder waarvoor het model is gekalibreerd, met name de overstromingsduur een grote invloed had op de LAl-ontwikkeling en daarmee op de

reflectie. 
Langs de inundatie- en competitiegradiënt in twee Nederlandse zoutmoerassen hebben we de reflectie in situ gemeten op blad- en gemeenschapsniveau. Op bladniveau zagen we dat de vegetatie-index NDVI redelijk goed de overstromingsstress voor twee pioniersoorten (Spartina en Scirpus) weergeeft, bij een van de onderzochte soorten (Spartina) vertegenwoordigde deze ook concurrentiestress. Op bladniveau vertoonde de REIP (Red Edge Inflection Point) veel variatie en was deze niet significant gerelateerd aan overstromingsduur of concurrentie. Op gemeenschapsniveau vonden we dat REIP index beter presteerde dan NDVI voor beide onderzoekssoorten. Dit geeft aan dat bij het kiezen van een vegetatie-index het meetniveau cruciaal is; een index die goed verband houdt met stress op bladniveau, heeft mogelijk geen duidelijke relatie met stress wanneer deze op gemeenschapsniveau wordt toegepast. Bij het toepassen van optische stressindicatoren op het riet in de Donaudelta ontdekten we dat optische vegetatie-indices slecht gerelateerd waren aan het uitzet- en terugtrekpatroon van het riet en de bijbehorende verwachte stress in rietplanten. In dit geval hielden radardata veel beter verband met de ontwikkelingspatronen in riet. Dit geeft aan dat stressdetectie complex is en zowel soort- als systeemspecifiek kan zijn. De keuze van de indicator hangt daarom af van hoe een stressfactor de vegetatie beïnvloedt en hoe dit op zijn beurt de reflectie en structuur beïnvloedt.

\section{Veerkracht als stressindicator}

Planten kunnen feedbacks gebruiken om hun omgeving te stabiliseren en hun stress te verminderen, maar wanneer deze feedbacks stoppen, kan het systeem plotseling instorten. Omdat feedbacks stress kunnen maskeren, zijn reguliere monitoringstechnieken mogelijk niet afdoende om stress in deze gevallen te detecteren. Om het naderen van een dergelijke situatie, waarin een systeem plotseling instort, te kunnen detecteren, zijn veerkrachtmetingen voorgesteld. Veerkracht kan worden gemeten door kunstmatig een verstoring te veroorzaken en de hersteltijd te meten. We hebben veerkrachtmetingen uitgevoerd langs een hoogtegradiënt en konden zo het effect van overstroming en concurrentiestress op veerkracht beoordelen. We vonden enig effect van inundatiestress, vooral op het herstel van de vegetatiebedekking, maar de resultaten verschilden tussen de twee geteste pioniersoorten (Spartina en Scirpus). Herstel van vegetatiehoogte was slechts gecorreleerd met inundatiestress voor één soort (Scirpus). De interpretatie van deze gegevens is complex, aangezien het herstel zowel de hergroei na de verstoring als mogelijke extra stressoren tijdens de herstelperiode vertegenwoordigt. Daarom is het gemete herstel eigenlijk de combinatie van het herstel van biomassa (hergroei) en het herstel van veerkracht (herstel van de vermogen van een plant om stress te weerstaan). Deze techniek zal verder moeten 
worden ontwikkeld voordat hij breed kan worden ingezet om stress of systeemstabiliteit te modelleren.

\section{Conclusie en aanbevelingen}

In deze studie hebben we methoden bekeken om stress in zoutmoerassen te monitoren, om zo het beheer te vergemakkelijken. Onze resulaten laten zien dat er geen enkele vegetatie-index is die in alle kwelders de stress goed weergeeft, en dat op radar gebaseerde indices in sommige systemen beter presteren dan op reflectie gebaseerde vegetatie-indices. Daarom zal stressmonitoring voorlopig het best gebiedsspecifiek toegepast kunnen worden, en hoewel remote sensing een waardevol instrument zal zijn voor deze analyses, zal het moeten worden toegepast door experts die de beperkingen van technieken begrijpen.

We pleiten voor een meer wetenschappelijk gebruik van vegetatiestructuurgegevens, zowel bij remote sensing als bij veldmetingen. Verdere ontwikkeling van deze technieken en een beter begrip van deze technieken zijn vereist voordat ze kunnen worden toegepast om het management te ondersteunen. Verder pleiten we voor voorzichtigheid bij het toepassen van herstel snelheid om de systeemstabiliteit of systeemstress te schatten.

Wij bevelen daarom aan kwelderbeheerders aan:

- Gebruik remote sensing om de stressontwikkeling in kwelders te volgen, maar zorg ervoor dat er een remote sensing deskundige en ecoloog bij betrokken zijn om een juist gebruik van de vegetatie-indices en hun interpretatie te verzekeren.

- Biomassa is nog steeds een betrouwbare manier om stress vast te stellen, maar vanwege het arbeidsintensieve karakter ervan moet het worden gereserveerd voor het monitoren van zeer kwetsbare of kritisch belangrijke systemen. Daarbij moet er rekening worden gehouden met mogelijke schade aan het systeem

- Streef ernaar het systeem als geheel te monitoren en veranderingen in het vegetatietype op te nemen. 
Wetenschappers willen we aanmoedigen om:

- managers te ondersteunen door te helpen bij het interpreteren van remote sensing gegevens;

- te onderzoeken hoe vegetatiestructuur kan worden opgenomen in monitoringschema's als een stressindicator;

- de herstel snelheid verder te valideren als potentieel hulpmiddel om stress in zoutmoerassen te schatten;

- door te gaan met het ontwikkelen van de tools die we hier presenteren, en deze uit te breiden met andere delen van een zoutmoeras, om zo brede ecosysteemmonitoring mogelijk te maken. 